\title{
Iron(III) Catalyzed Chain Growth Reactions of Polymeric Methylene Diphenyl Diisocyanate
}

\author{
Anthony P. Gies*, Zdravko Stefanov, Nathan J. Rau, Debashis Chakraborty, \\ Praveenkumar Boopalachandran, and J. Paul Chauvel
}

Department of Core R\&D Analytical Sciences, The Dow Chemical Company, 2301 N. Brazosport Blvd., B-1820, Freeport, TX 77541

Macromolecules: September 08, 2015

TITLE RUNNING HEAD: Chain Growth Reactions of PMDI.

CORRESPONDING AUTHOR FOOTNOTE: Address reprint requests to Anthony P. Gies, Department of Core R\&D Analytical Sciences, The Dow Chemical Company, 2301 N. Brazosport Blvd., B-1219, Freeport, TX 77541. Telephone: (979) 238-1778. E-mail: APGies@,Dow.com 


\section{FIGURE CAPTIONS:}

Figure S1. MALDI-TOF/TOF CID fragmentation spectrum of the protonated $471.5 \mathrm{Da}$ carbodiimide precursor ion (Structure 1-2M).

Figure S2. MALDI-TOF/TOF CID fragmentation spectrum of the protonated 1119.4 Da carbodiimide dimer precursor ion (Structure 1-4).

Figure S3. MALDI-TOF/TOF CID fragmentation spectrum of the protonated 803.3 Da methanol-capped uretonimine branched carbodiimide $(I C D)$ precursor ion (Structure 1$5)$.

Figure S4. MALDI-TOF/TOF CID fragmentation spectrum of the protonated $725.3 \mathrm{Da}$ hydroxyl-containing uretonimine branched carbodiimide precursor ion (Structure 1-5Hy).

Figure S5. MALDI-TOF/TOF CID fragmentation spectrum of the sodiated $843.3 \mathrm{Da}$ methanol-capped hydroxyl-containing uretonimine branched carbodiimide precursor ion (Structure 1-5Hy).

Figure S6. MALDI-TOF/TOF CID fragmentation spectrum of the protonated 1085.4 Da methanol-capped imino-s-triazine branched carbodiimide (1CT) precursor ion (Structure $1-6)$.

Figure S7. MALDI-TOF/TOF CID fragmentation spectrum of the protonated $757.3 \mathrm{Da}$ biphenyl-containing guanidine branched carbodiimide precursor ion (Structure 1-7P).

Figure S8. MALDI-TOF/TOF CID fragmentation spectrum of the protonated 887.3 Da guanidine branched carbodiimide precursor ion (Structure 1-8). 
Figure S9. FTIR spectrum of the unheated "neat" PMDI, provide by Praveenkumar Boopalachandran (The Dow Chemical Company).

Figure S10. Stacked FTIR spectra of "neat" PMDI and PMDI heated with the presence of 2110 ppm (Sample B) and 214 ppm (Sample C) iron(II) chloride. These spectra were provided by Praveenkumar Boopalachandran (The Dow Chemical Company).

Figure S11. FTIR MALDI-TOF mass spectrum of PMDI side products formed in the presence of iron(II) chloride (2110 ppm Fe(II): Sample B), covering the mass range of 300-2600 Da.

Figure S12. MALDI-TOF mass spectrum of PMDI side products formed in the presence of of low levels of iron(II) chloride (214 ppm Fe(II): Sample C), covering the mass range of 300-2600 Da.

Figure S13. MALDI-TOF mass spectrum of PMDI side products formed in the presence of low levels of iron(III) chloride (169 ppm Fe(III): Sample D), covering the mass range of 300-2600 Da. 
Figure S1.

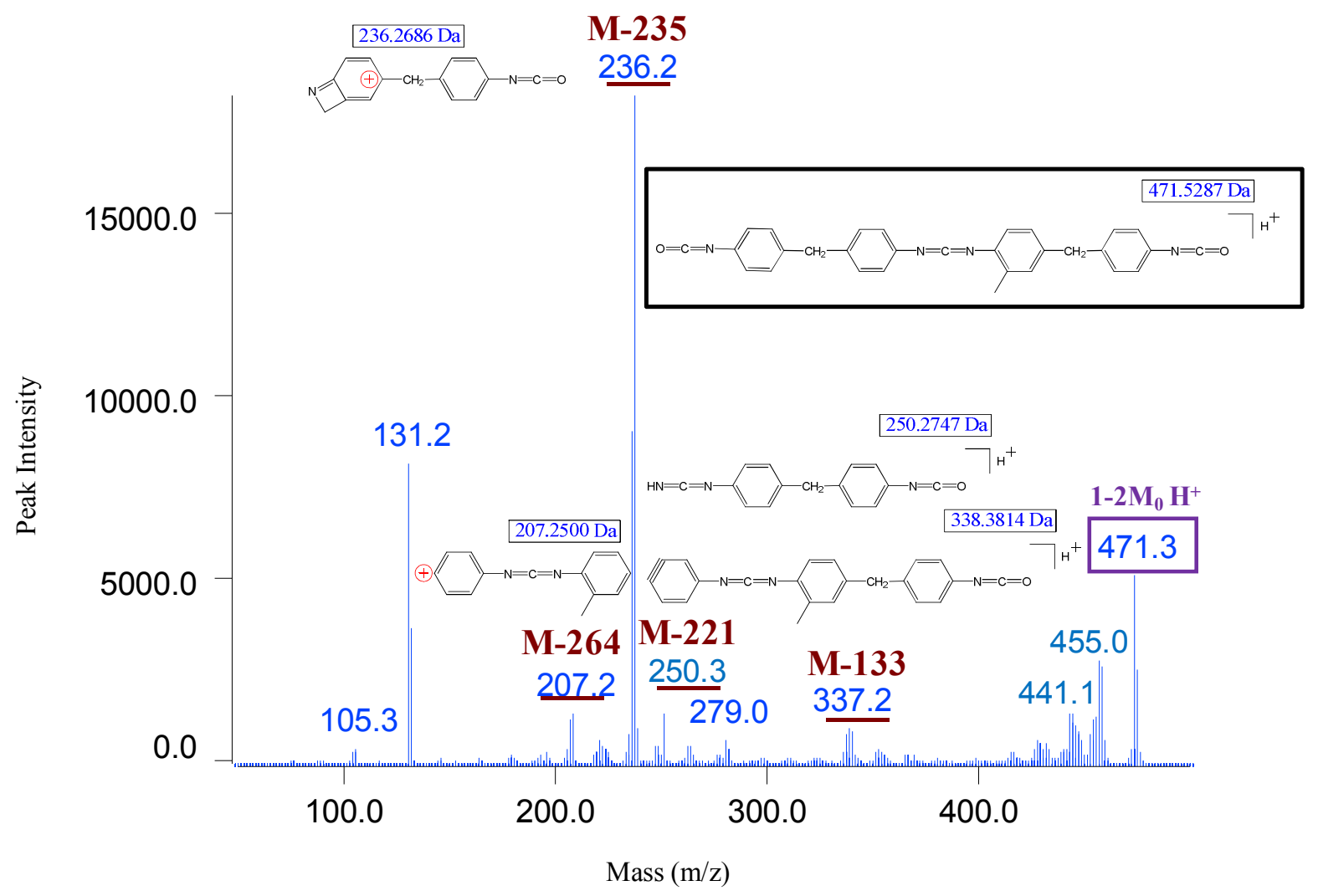

Figure S2.

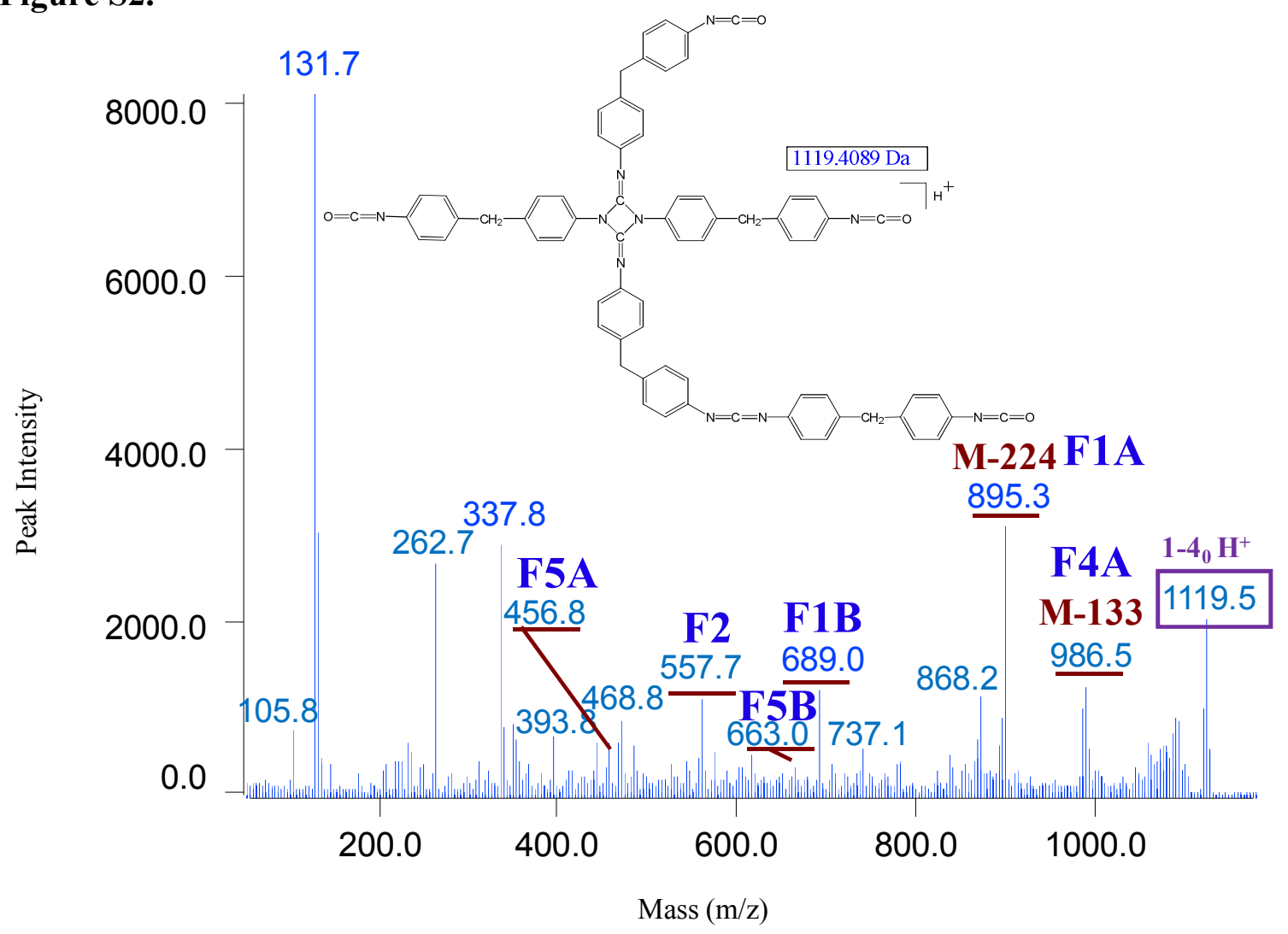


Figure S3.

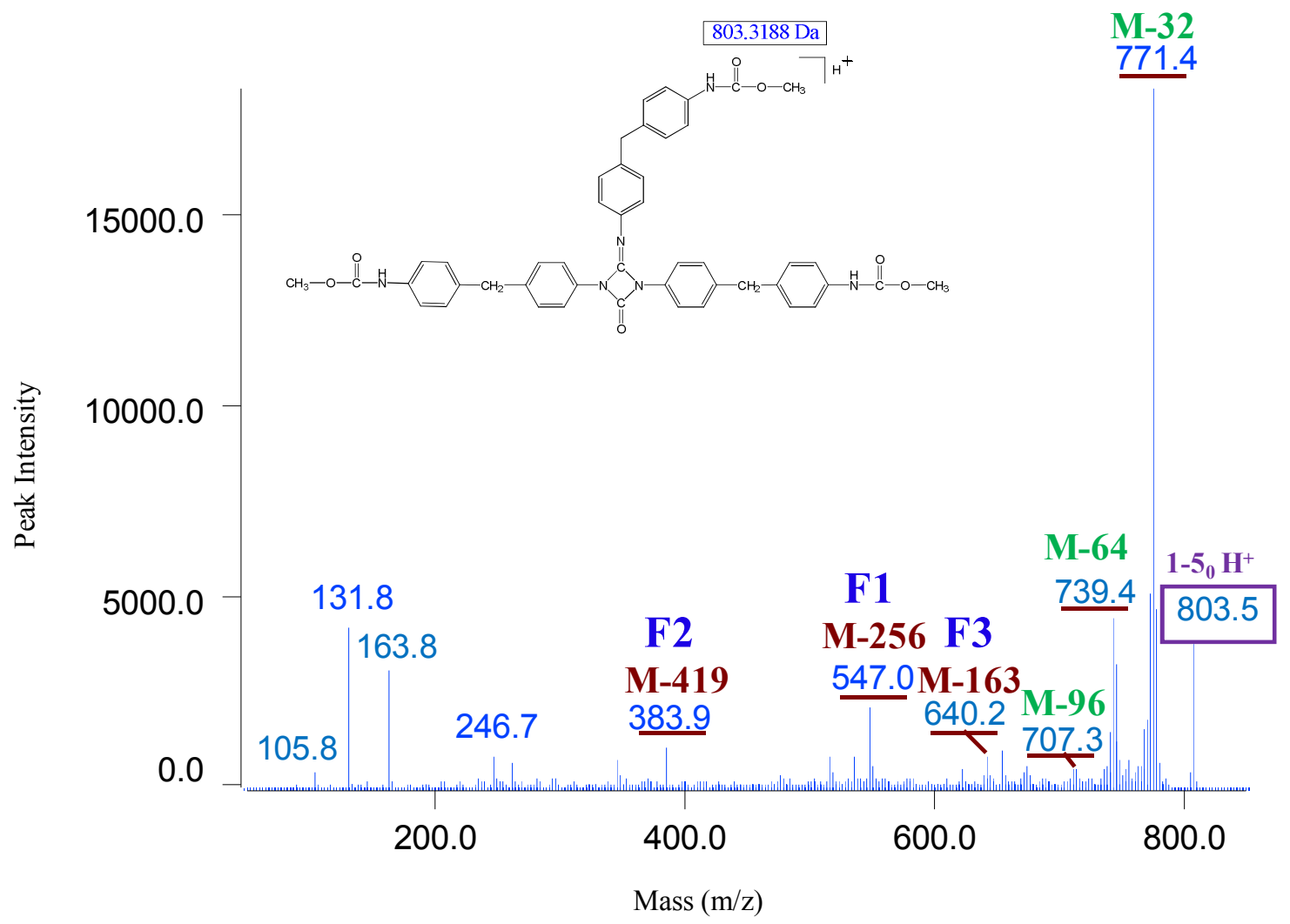

Figure S4.

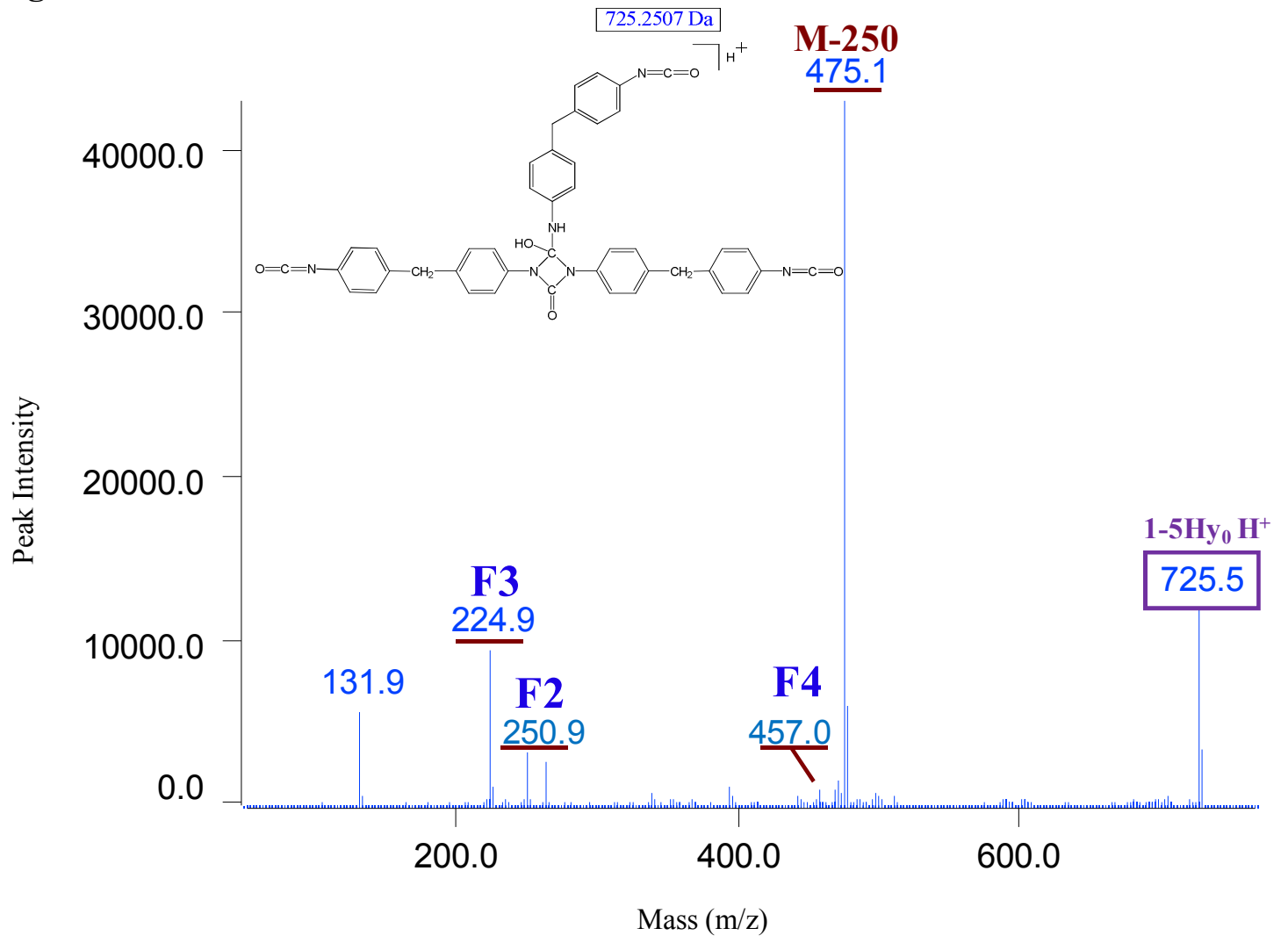


Figure S5.

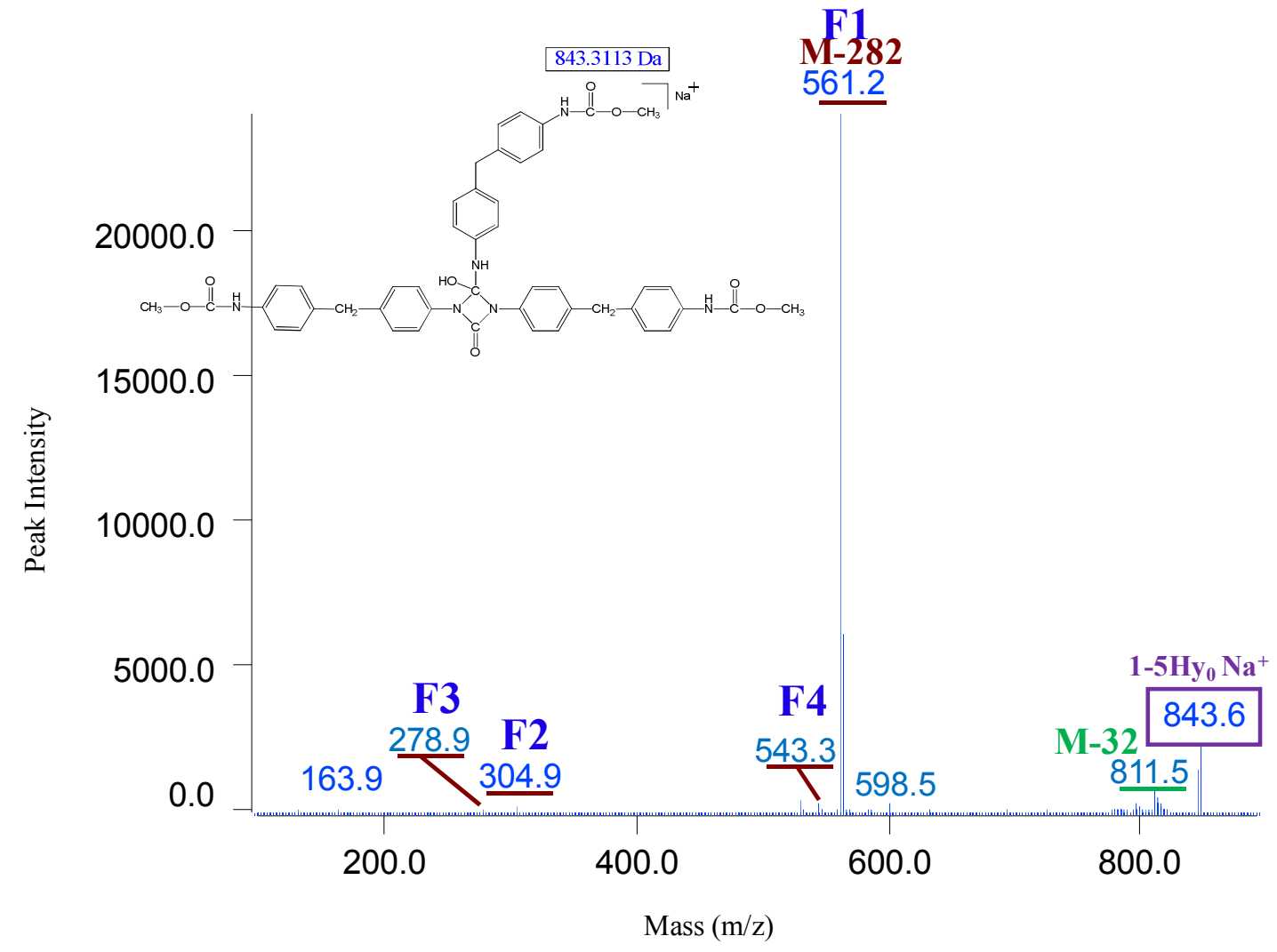

Figure S6.

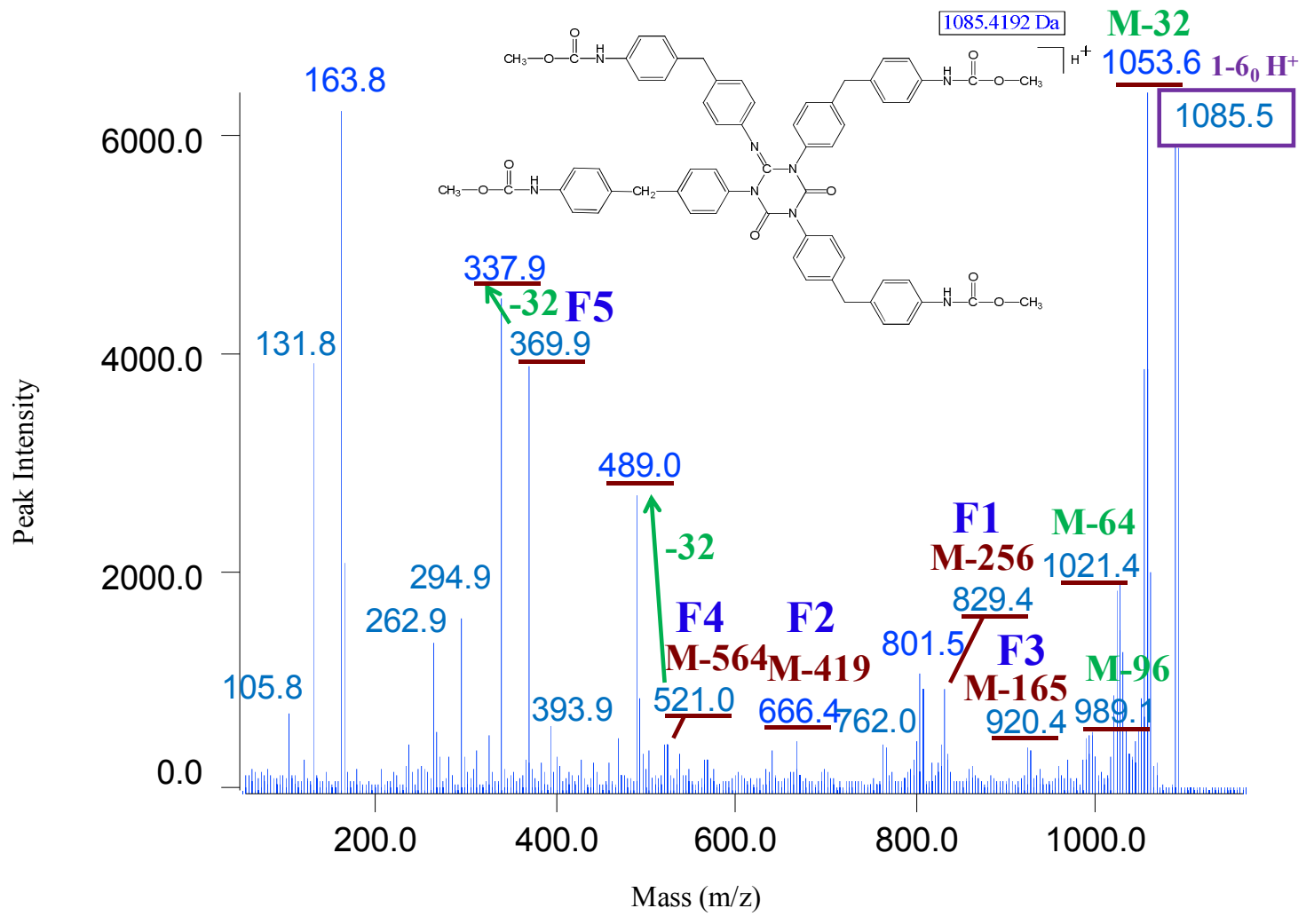


Figure S7.

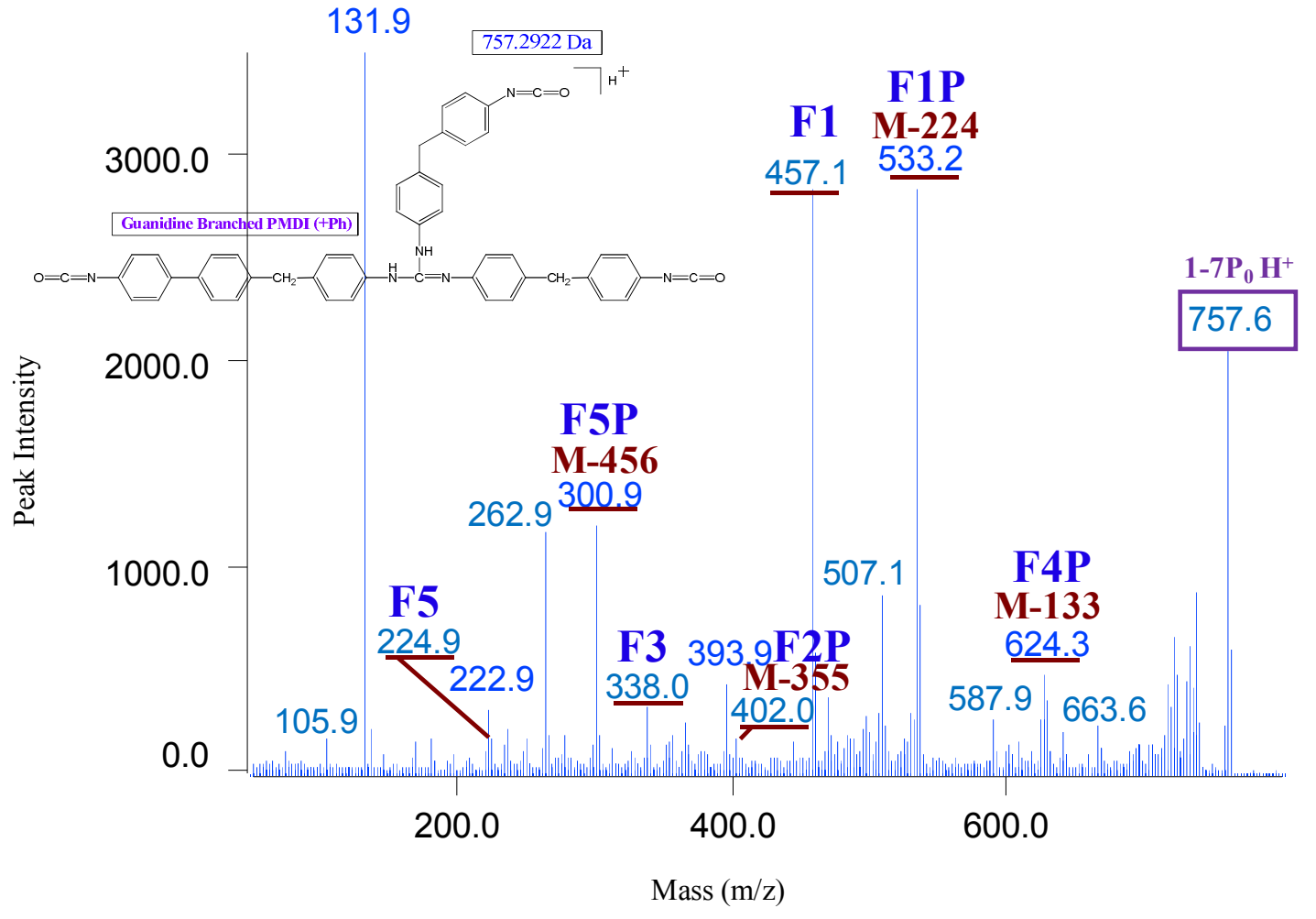

Figure S8.

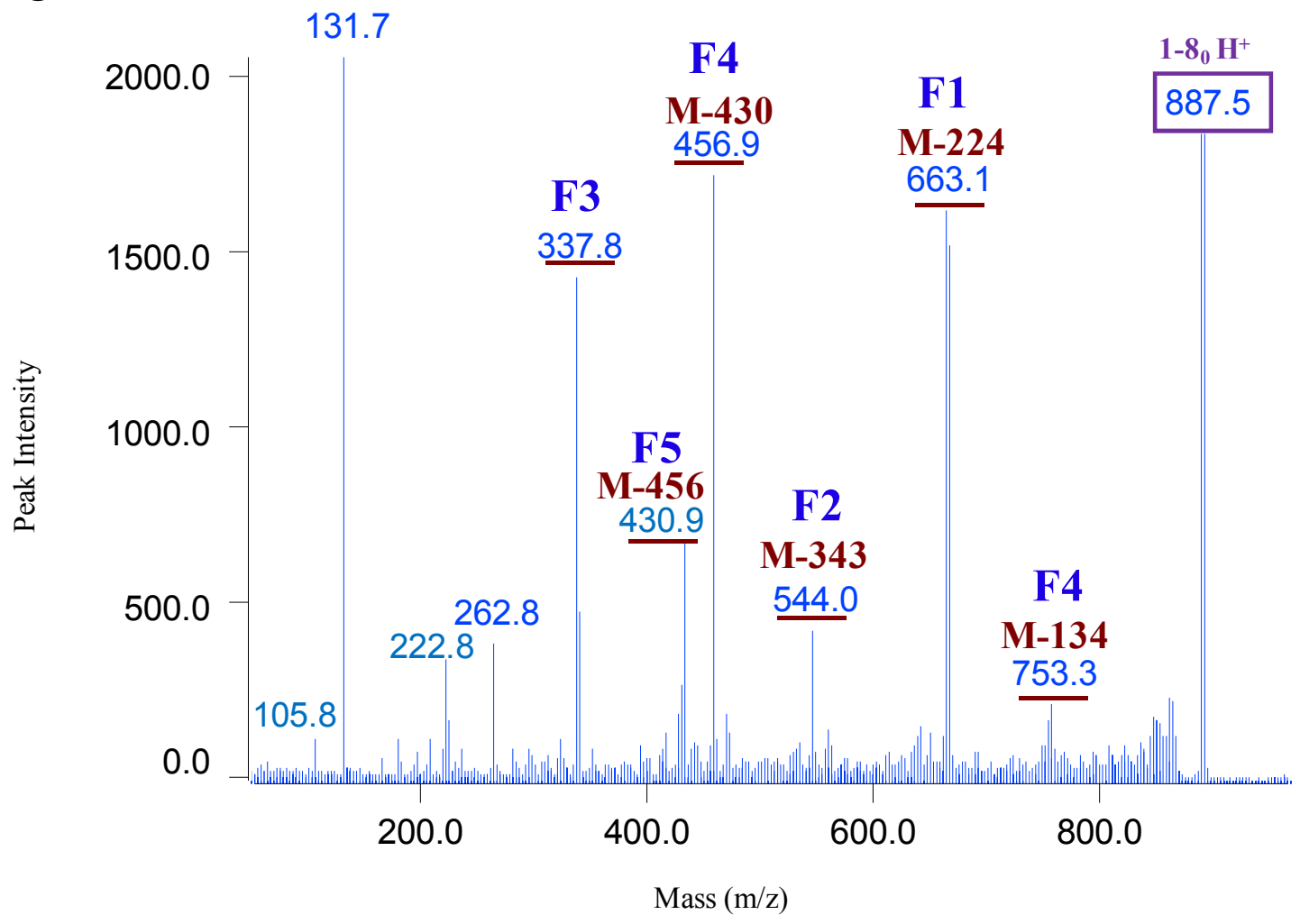


Figure S9.

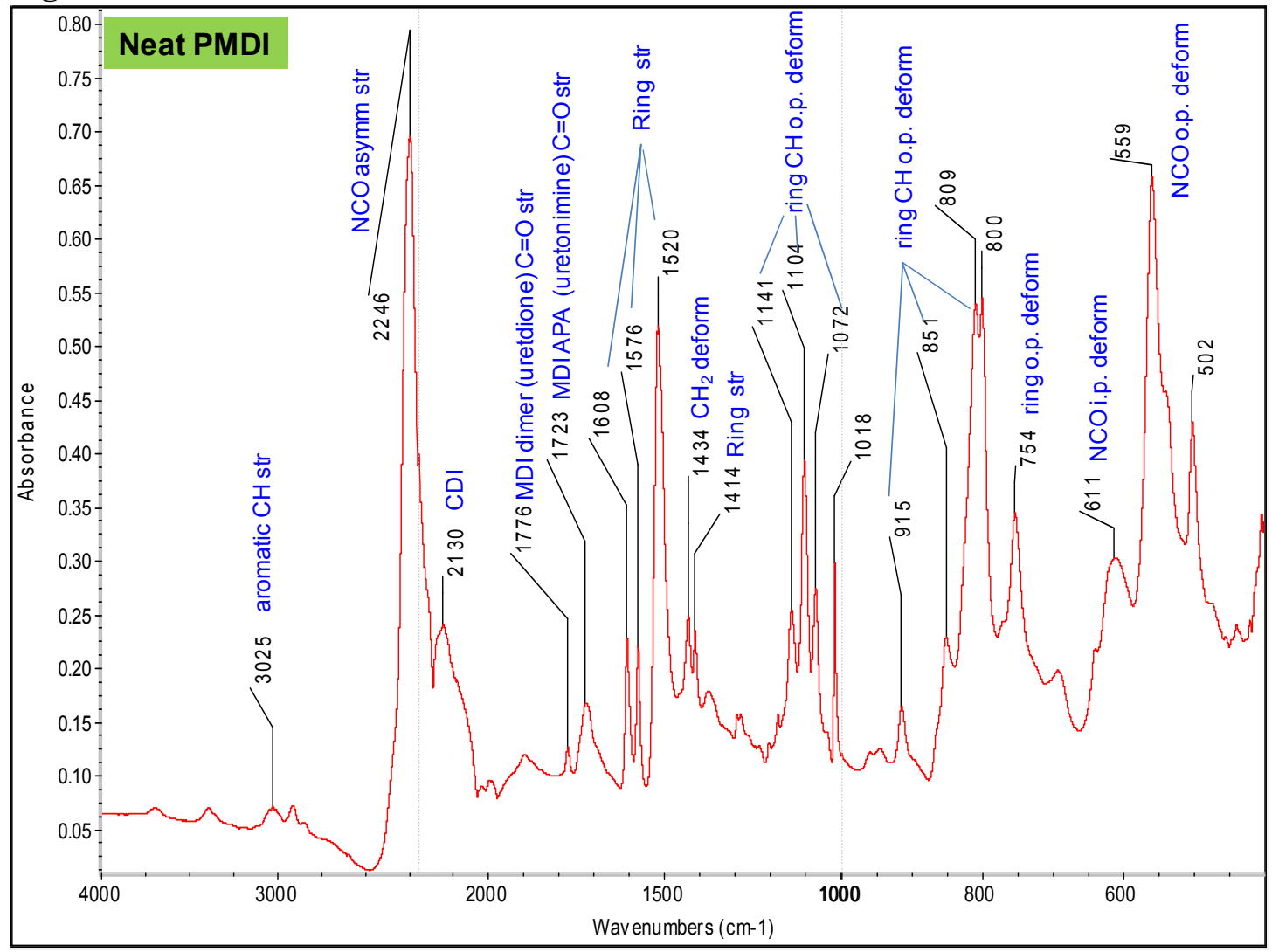

Figure S10.

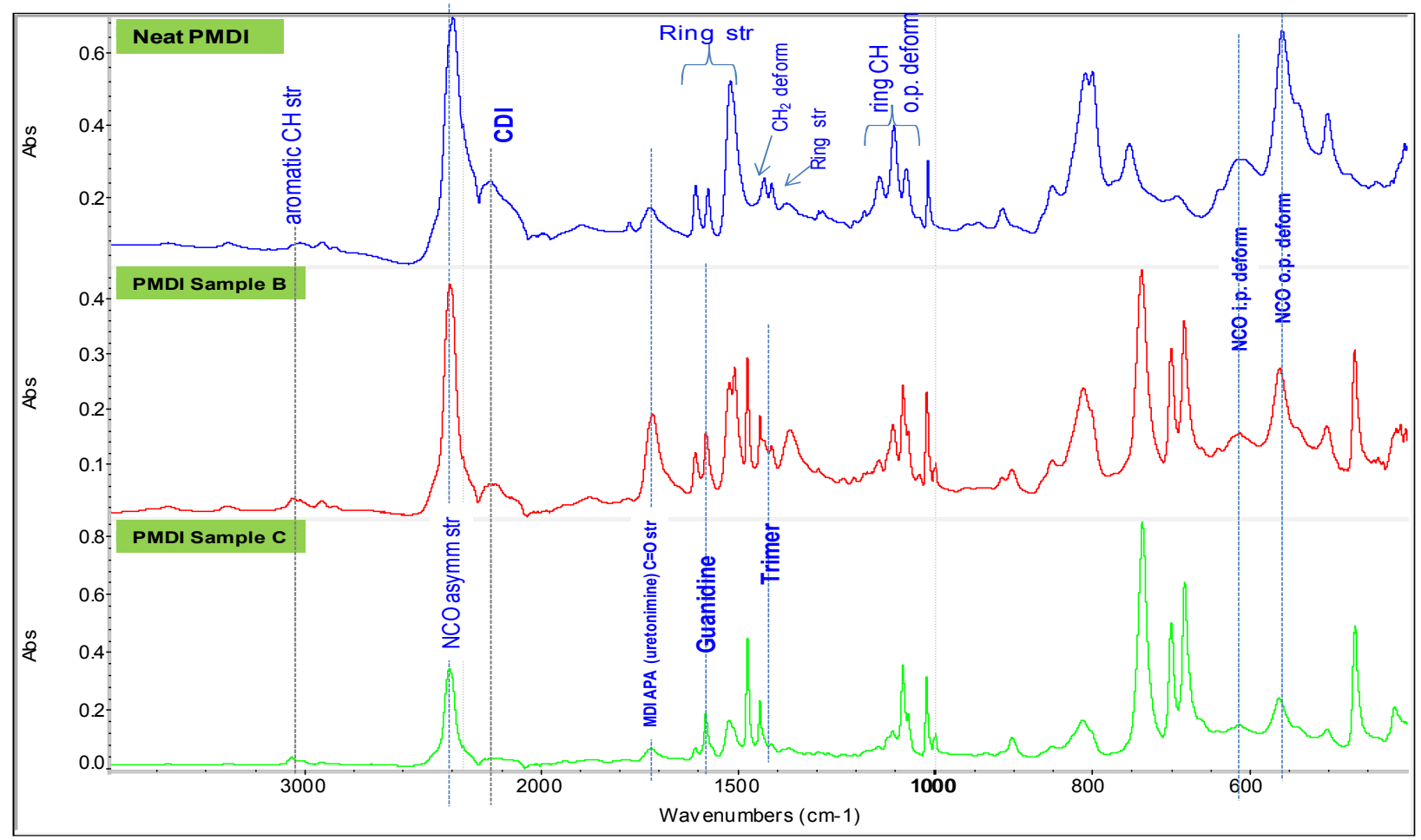


Figure S11.

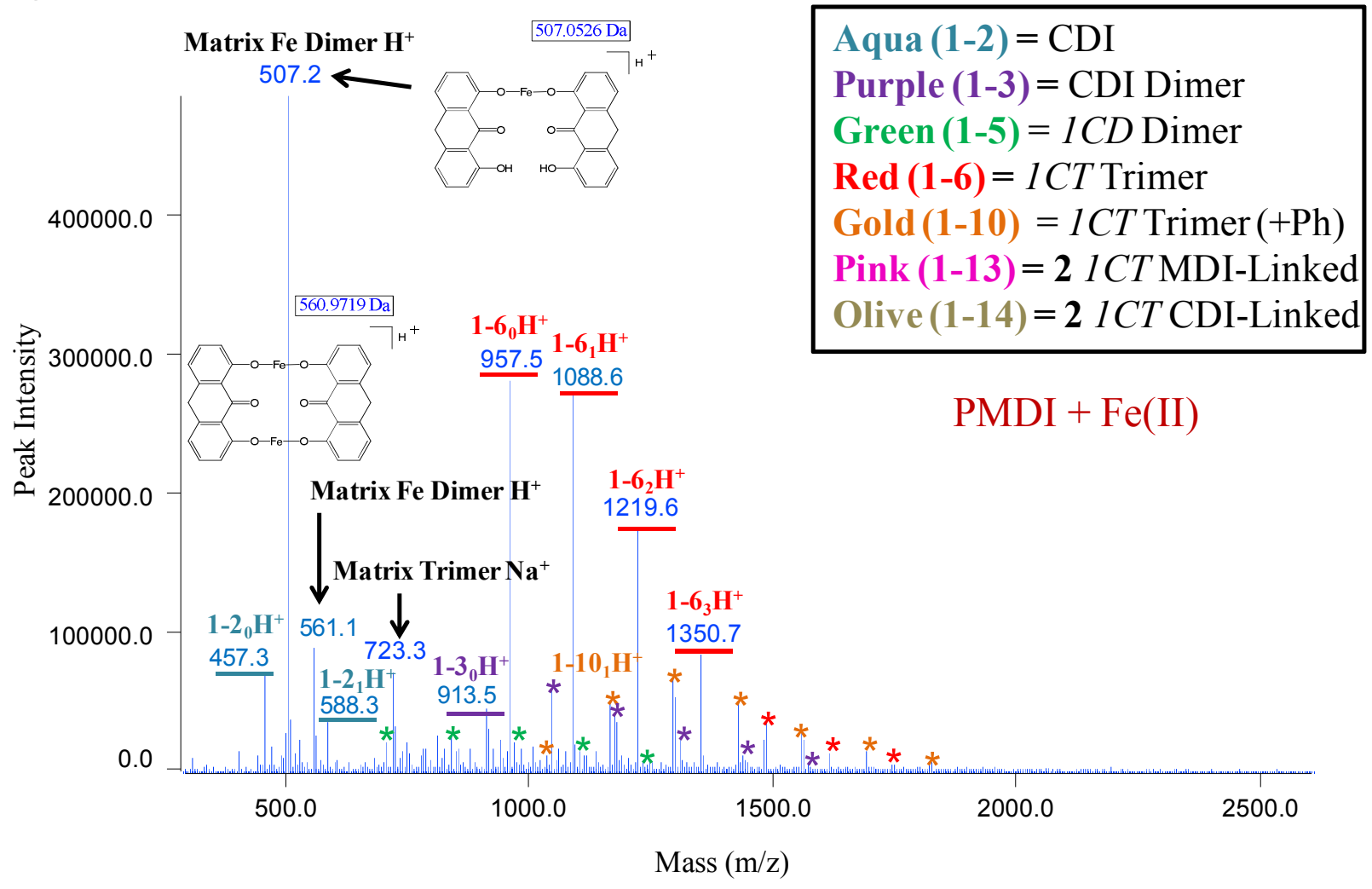

Figure S12.

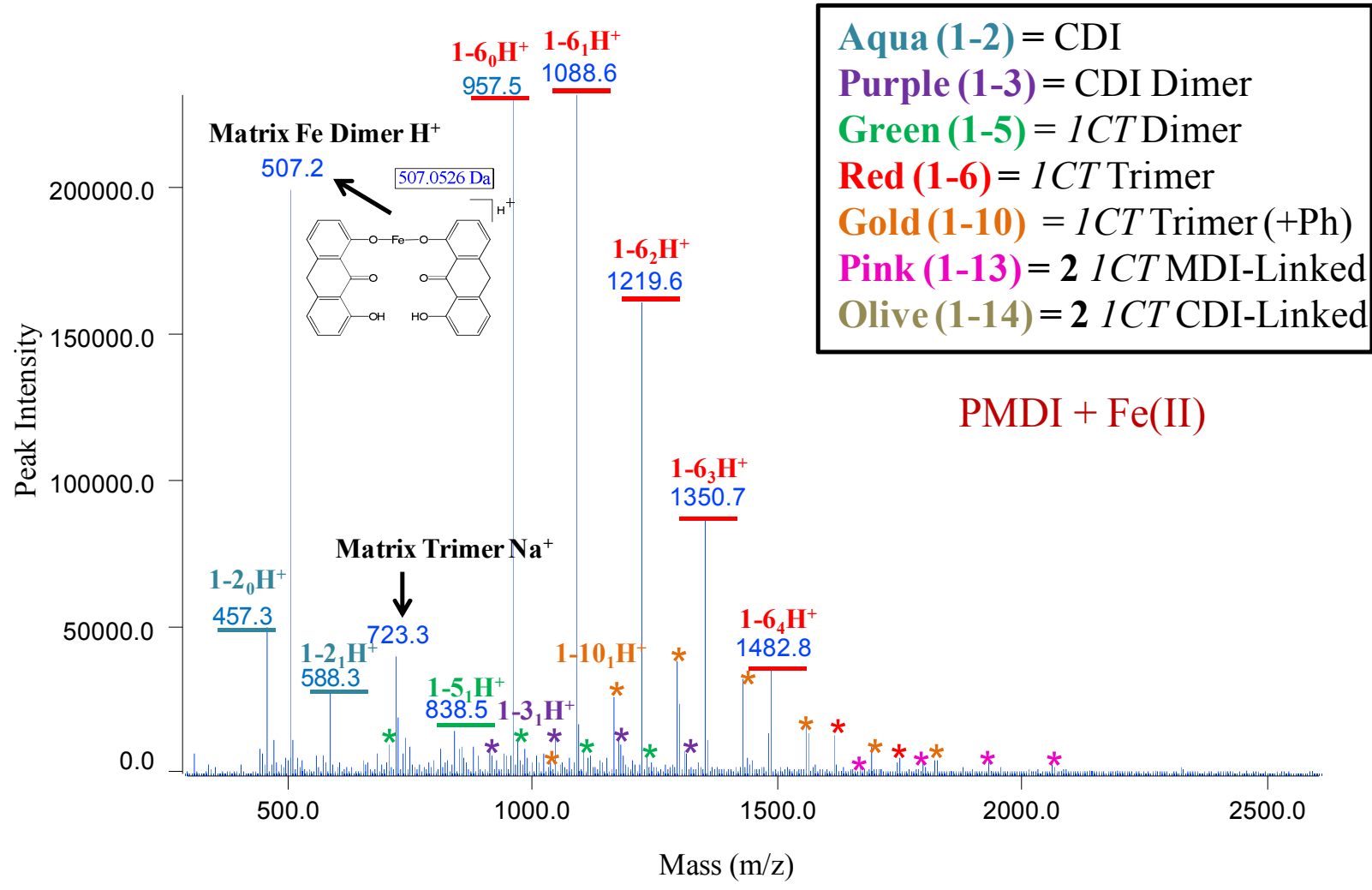


Figure S13.

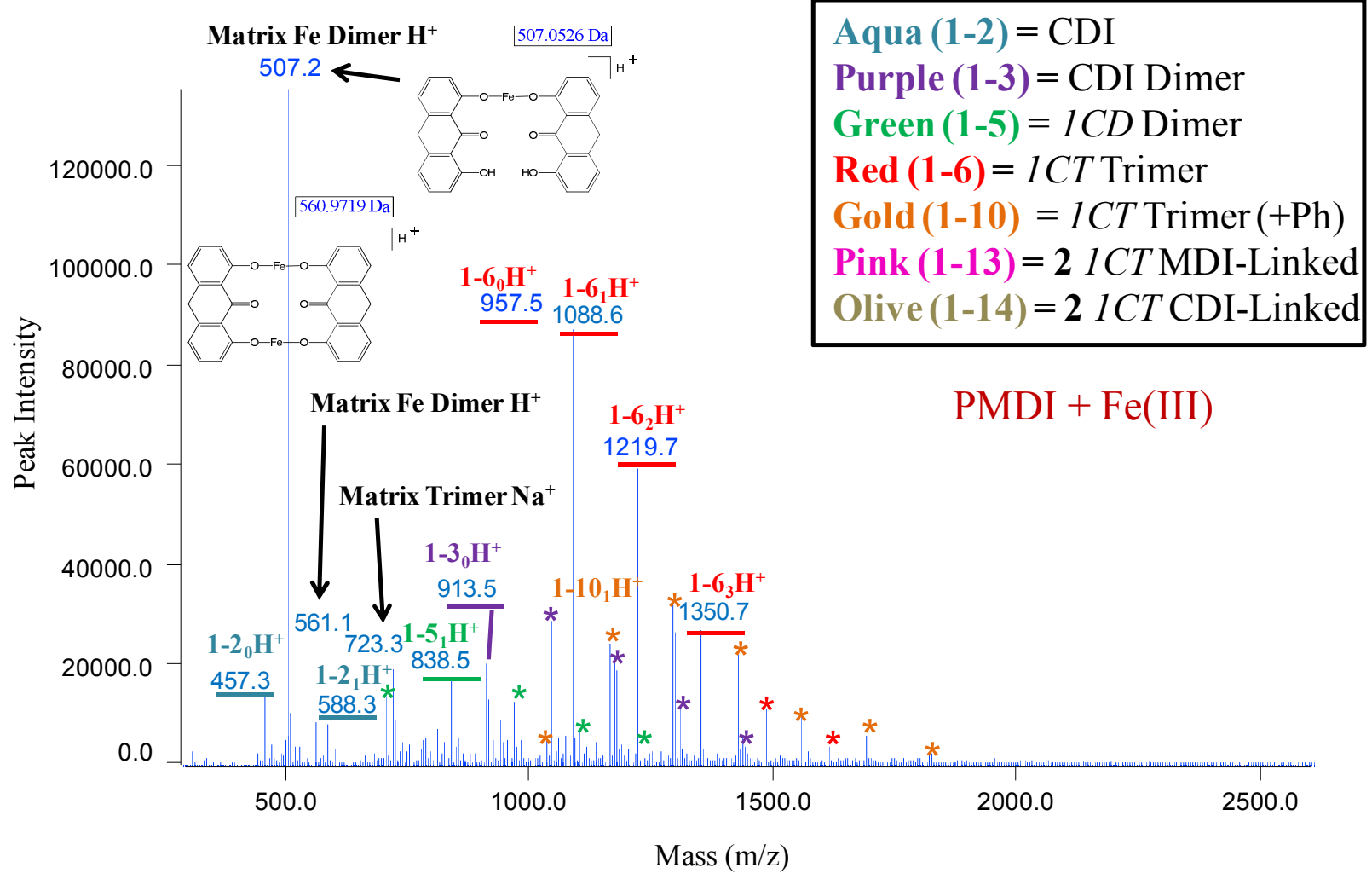




\section{SCHEME TITLES:}

Scheme S1. Low kinetic energy fragmentation pathways of the protonated 1119.4 Da carbodiimide dimer precursor ion (Structure 1-4).

Scheme S2. Low kinetic energy fragmentation pathways of the protonated 803.3 Da methanol-capped uretonimine branched carbodiimide precursor ion (ICD) (Structure 1$5)$.

Scheme S3. Low KE fragmentation pathways of the protonated 725.3 Da hydroxylcontaining uretonimine branched carbodiimide precursor ion (Structure 1-5Hy).

Scheme S4. Low KE fragmentation pathways of the sodiated 843.3 Da methanol-capped hydroxyl-containing uretonimine branched carbodiimide precursor ion (Structure 1-5Hy).

Scheme S5. Low KE fragmentation pathways of the protonated 1085.4 Da methanolcapped imino-s-triazine branched carbodiimide (ICT) precursor ion (Structure 1-6).

Scheme S6. Low KE fragmentation pathways of the protonated 757.3 Da biphenylcontaining guanidine branched carbodiimide precursor ion (Structure 1-7P).

Scheme S7. Low KE fragmentation pathways of the protonated 887.3 Da guanidine branched carbodiimide precursor ion (Structure 1-8). 


\section{Scheme S1.}

A.

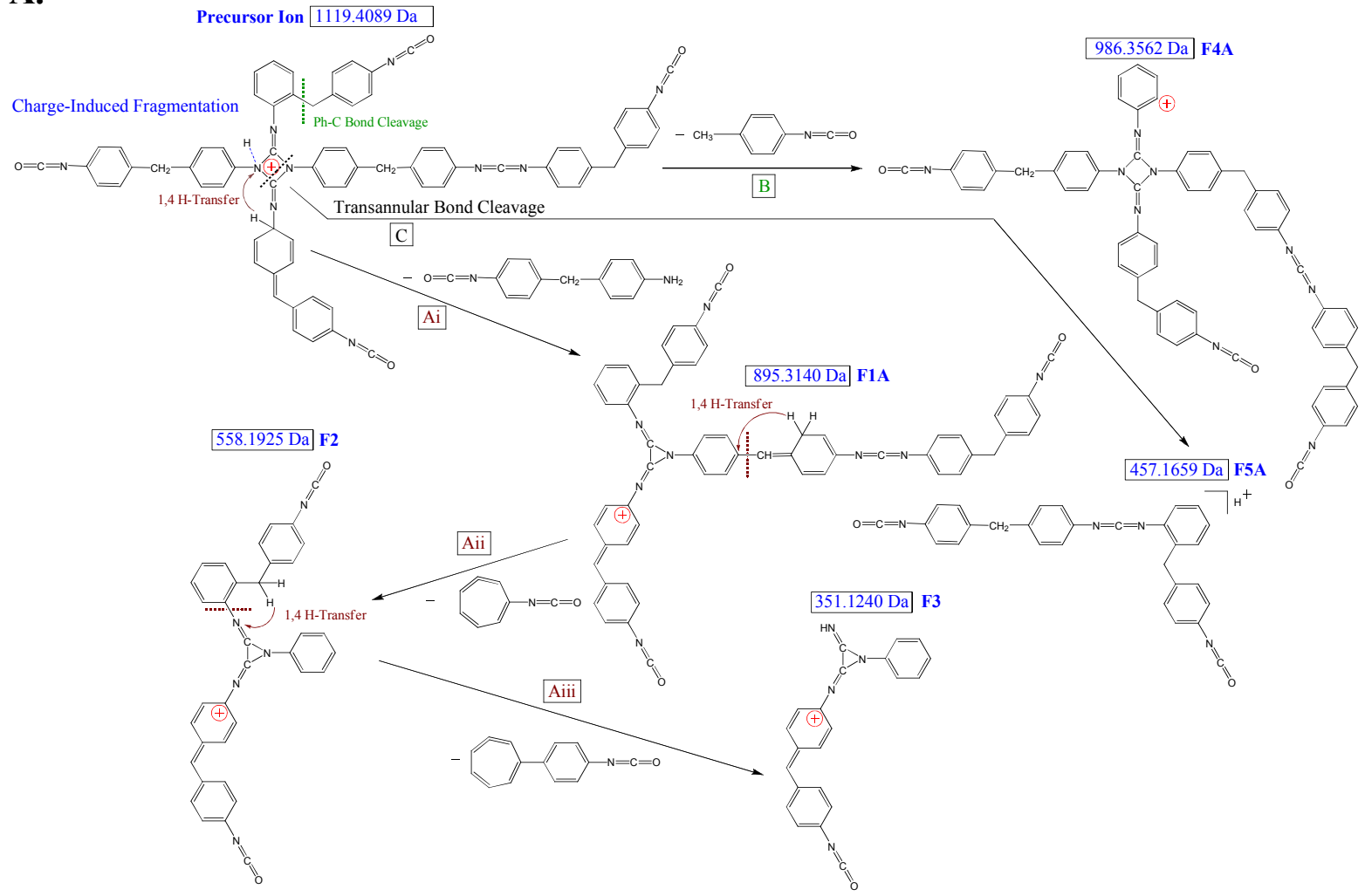

B.

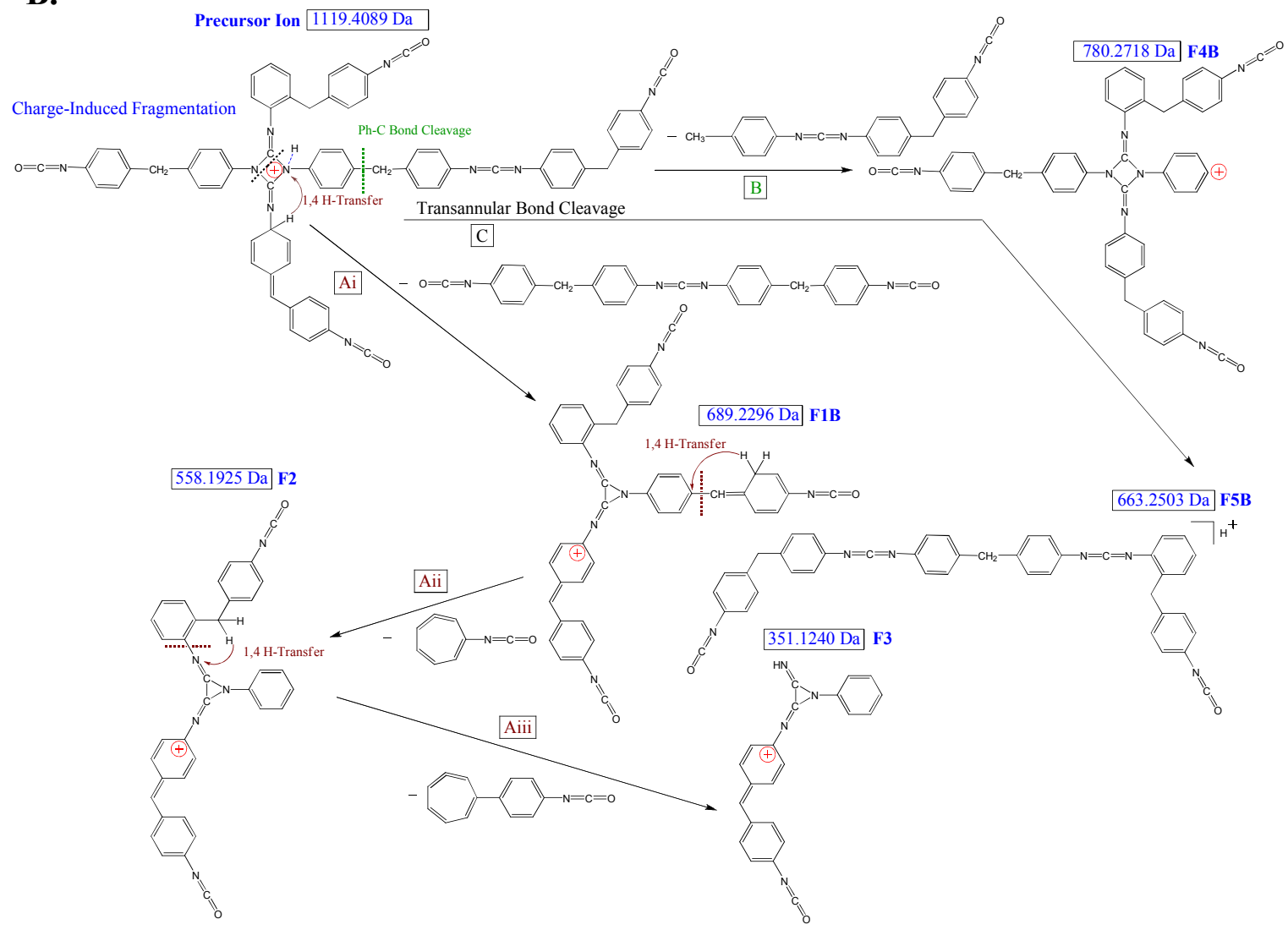




\section{Scheme S2.}

Precursor Ion 803.3188 Da

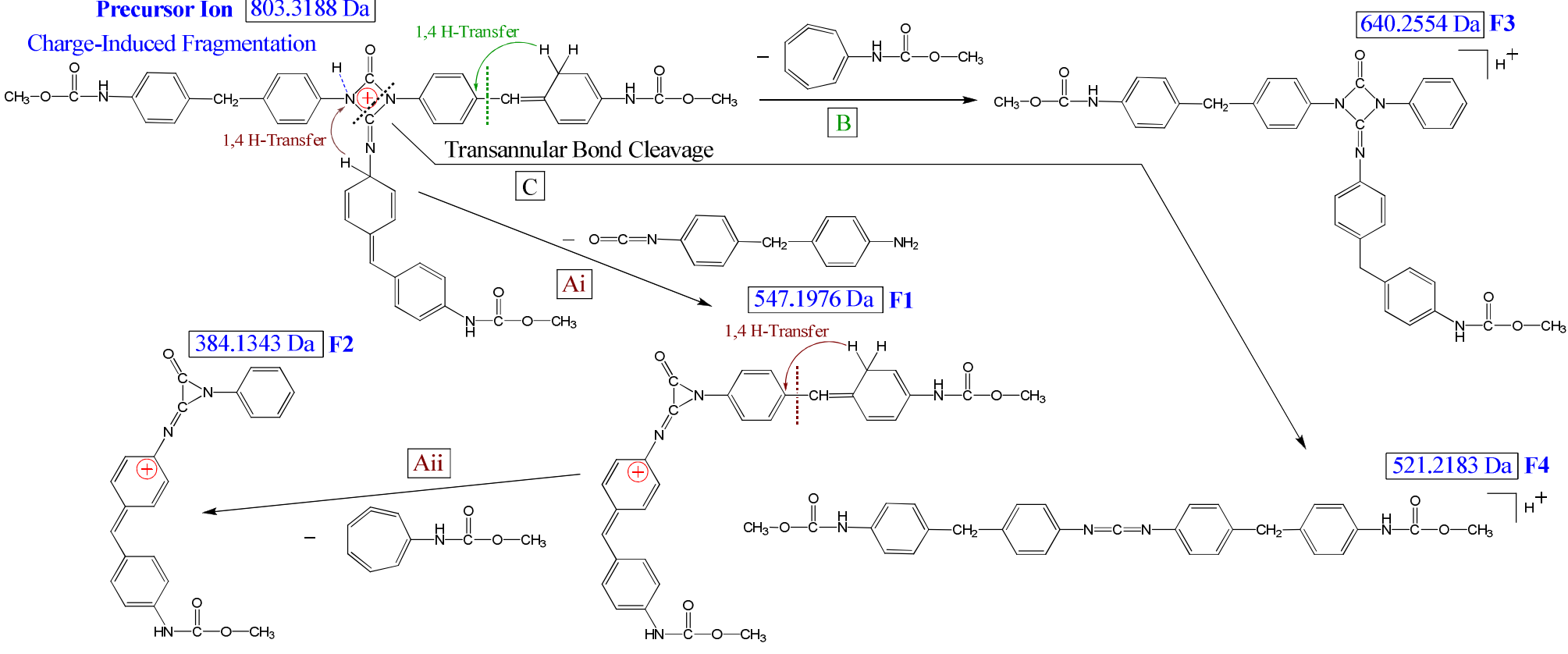

Scheme S3.

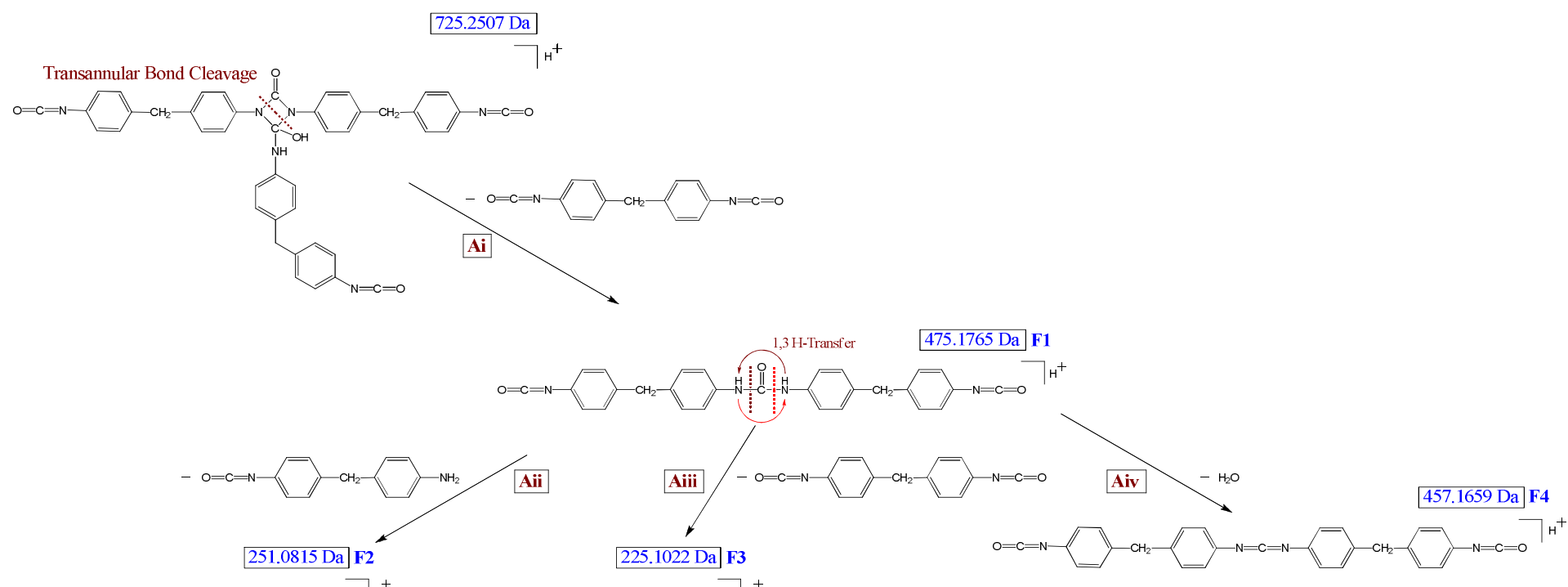




\section{Scheme S4.}

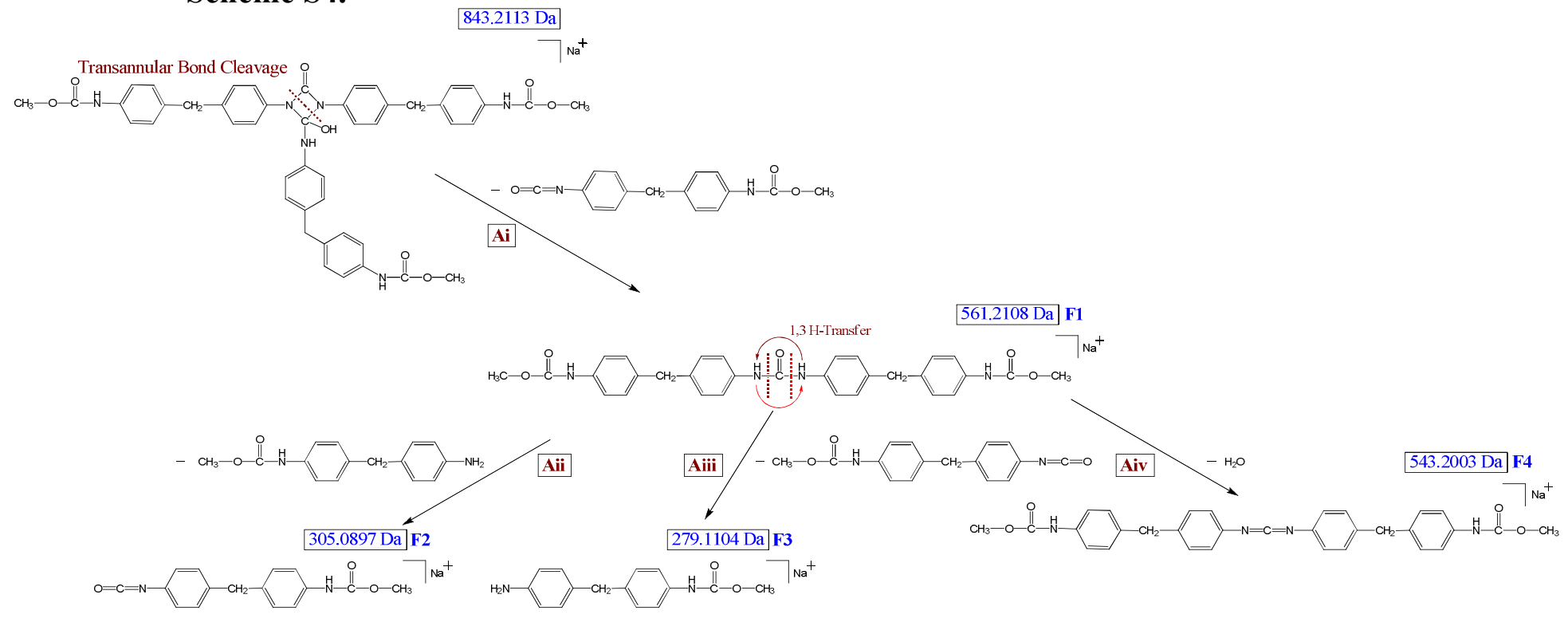

\section{Scheme S5.}

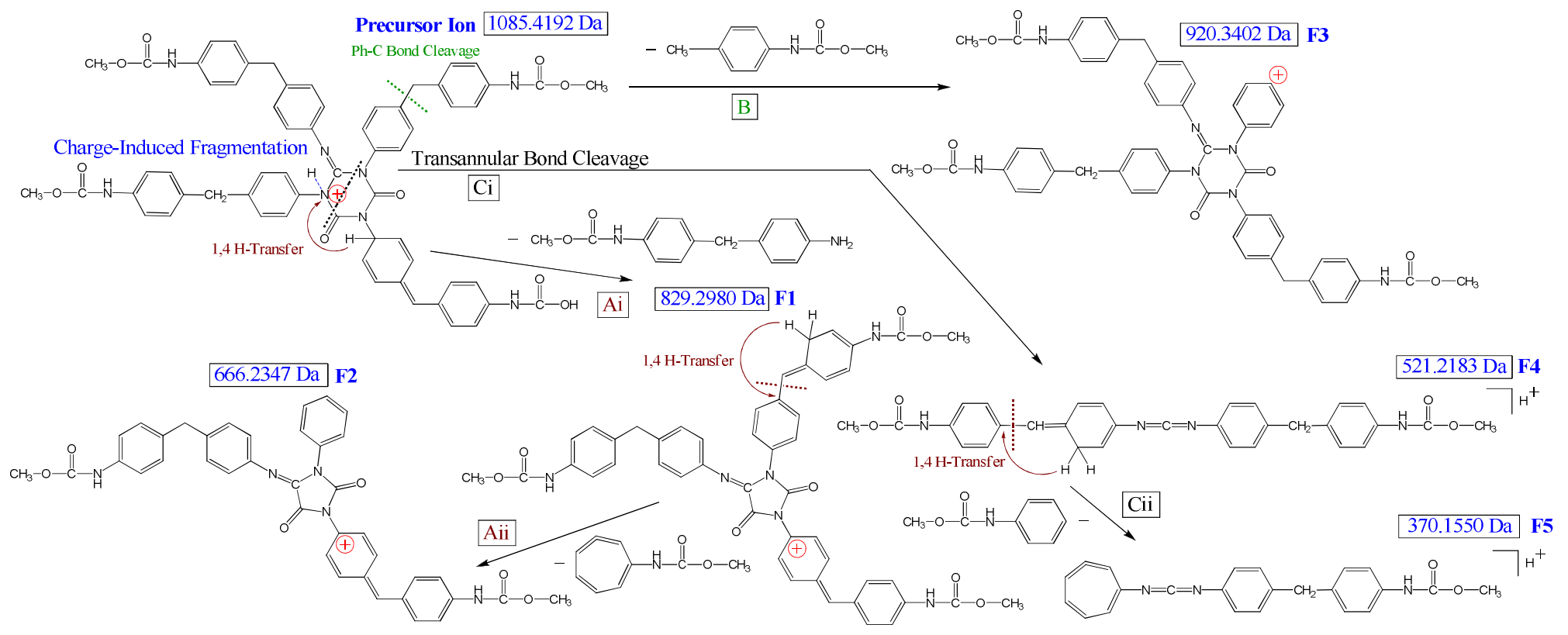




\section{Scheme S6.}

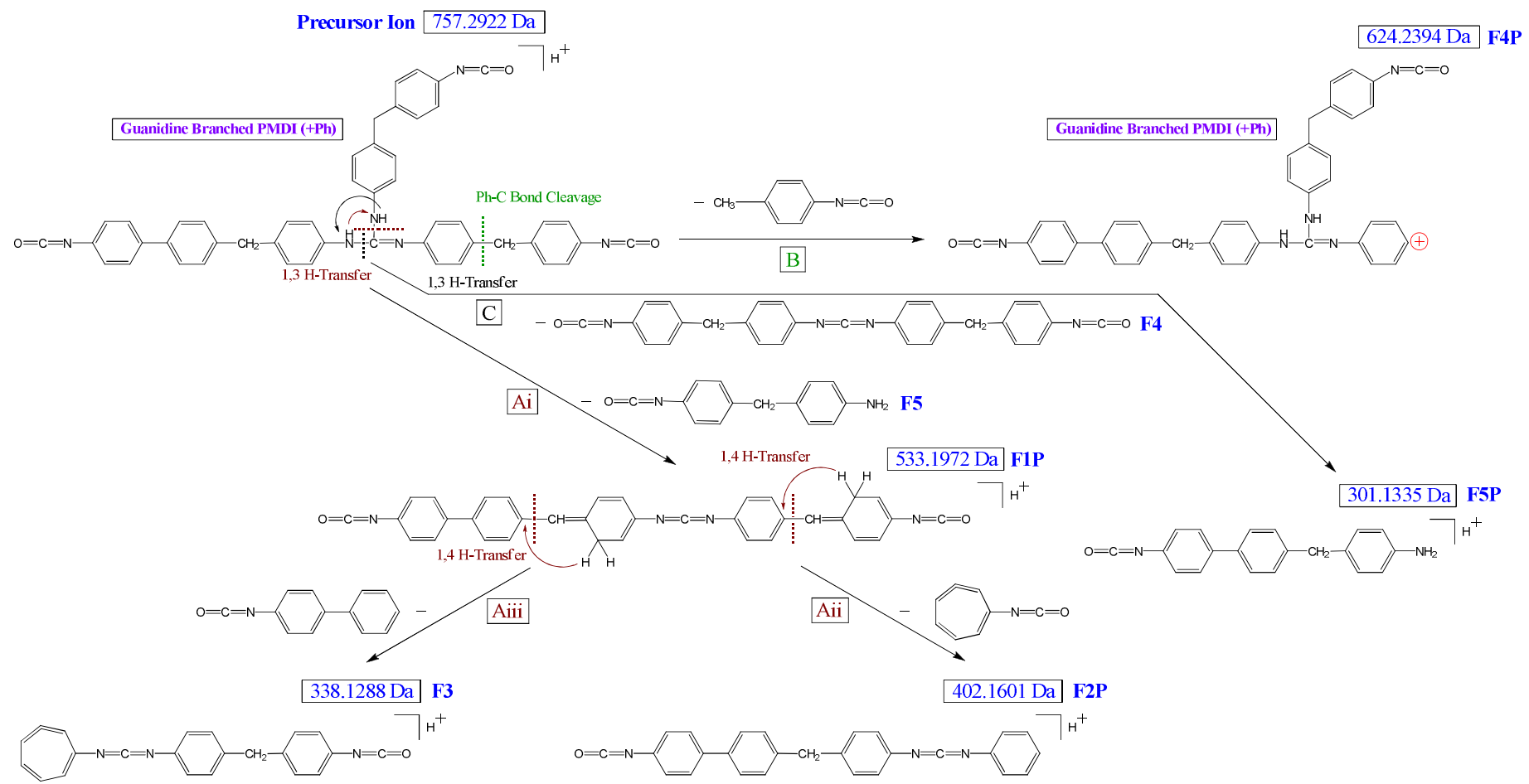

\section{Scheme S7.}

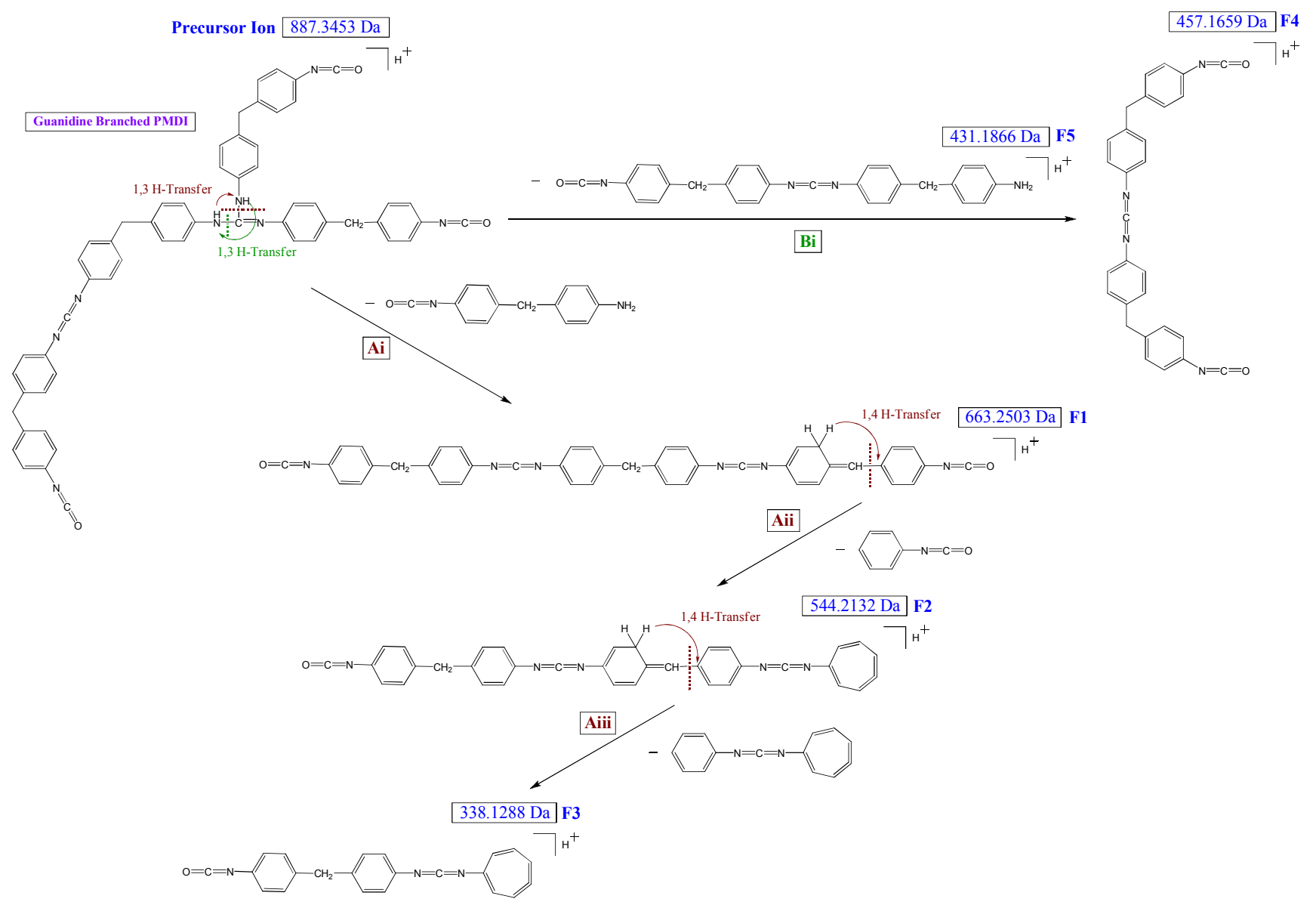


TABLES:

Table S1. PMDI aging experimental conditions. 
Table S1.

\begin{tabular}{|l|r|r|r|r|r|r|r|r|}
\hline Sample & PMDI, g & $\mathrm{FeCl}_{2}, \mathrm{~g}$ & $\mathrm{FeCl}_{3}, \mathrm{~g}$ & $\mathrm{CaO}, \mathrm{g}$ & $\mathrm{Fe}(\mathrm{II}), \mathrm{ppm}$ & $\mathrm{Fe}(\mathrm{III}), \mathrm{ppm}$ & Temperature, deg. C & Viscosity at 25 C, cps \\
\hline $\mathrm{A}$ & 6.93 & 0 & 0 & 1.067 & 0 & 0 & 180 & 37 \\
\hline $\mathrm{B}$ & 6.25 & 0.0297 & 0 & 1.067 & 2110 & 0 & 180 & 86 \\
\hline $\mathrm{C}$ & 6.224 & 0.003 & 0 & 1.1196 & 214 & 0 & 180 & 78 \\
\hline $\mathrm{D}$ & 6.0936 & 0 & 0.003 & 1.3303 & 0 & 169 & 180 & 89 \\
\hline $\mathrm{E}$ & 5.9909 & 0 & 0.0304 & 1.138 & 0 & 1746 & 180 & 149 \\
\hline
\end{tabular}




\section{Computational Supporting Information}

The main objective of the computational work supporting this study was to explore how $\mathrm{FeCl}_{3}$ affects the structure and energetics of the carbodiimide bond. We found that adduct formation is exothermic both in vacuum and in a simulated aromatic (benzene) solvent. The simulated benzene solvent models the environment of neat pMDI. In the resulting adduct, $\mathrm{FeCl}_{3}$ prefers to associate closely and further polarizes the carbodiimide bond. Overall, adduct formation with $\mathrm{FeCl}_{3}$ causes the carbodiimide bond to become activated towards reactions with either Lewis acids or bases (including dimerization).

\section{Calculation of Thermochemistry of Adduct Formation $\left(\Delta_{\mathrm{add}} G\right)$}

To investigate whether adduct formation is spontaneous we directly calculated the energy of toluene carbodiimide and $\mathrm{FeCl}_{3}$ individually (infinitely separated) and then calculated the energy of the adduct. $\Delta_{\text {add }} \mathrm{G}$ is calculated as the difference of the adduct minus the individual toluene carbodiimide and $\mathrm{FeCl}_{3}$ molecules (see below).

$\Delta_{\text {add }} \mathrm{G}=$ Adduct $-\left(\right.$ toluene carbodiimide $\left.+\mathrm{FeCl}_{3}\right)$

Calculated Energies in Hartrees (zero-point corrected)

vacuum in benzene

$\mathrm{FeCl} 3$

$-2644.34828-2644.3631$

Toluene Carbodiimide

$-689.296156-689.31655$

FeCl3_Carbodiimide Adduct

$-3333.680246-3333.7111$

Adduct Formation (kcal/mol)

$-22.5$

Gaussian Output Geometries and NPA Population Analyses

\section{$\underline{\mathrm{FeCl}_{3}} \underline{\text { Vacuum }}$}

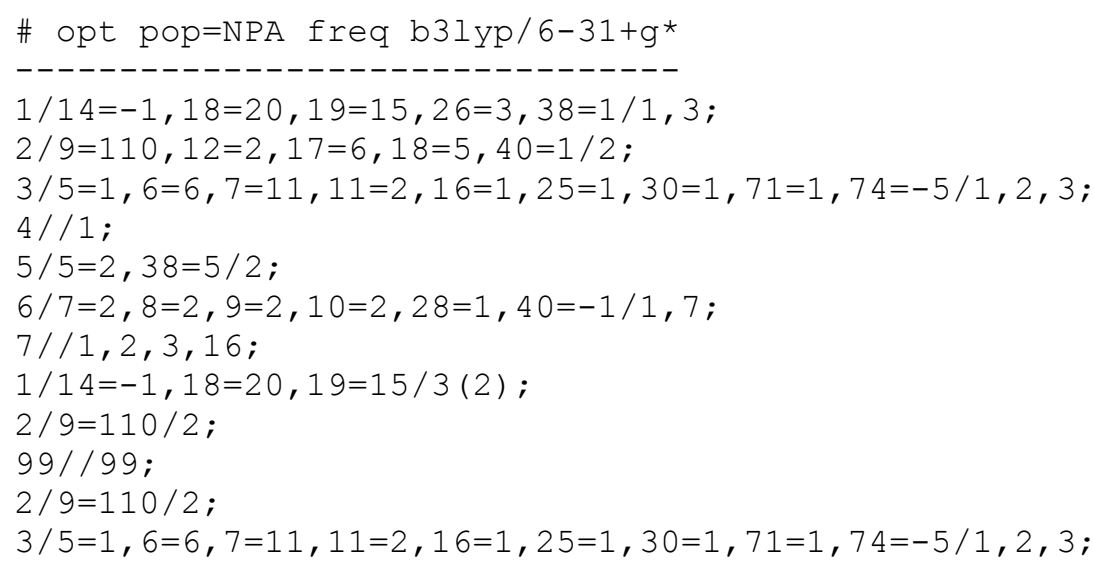




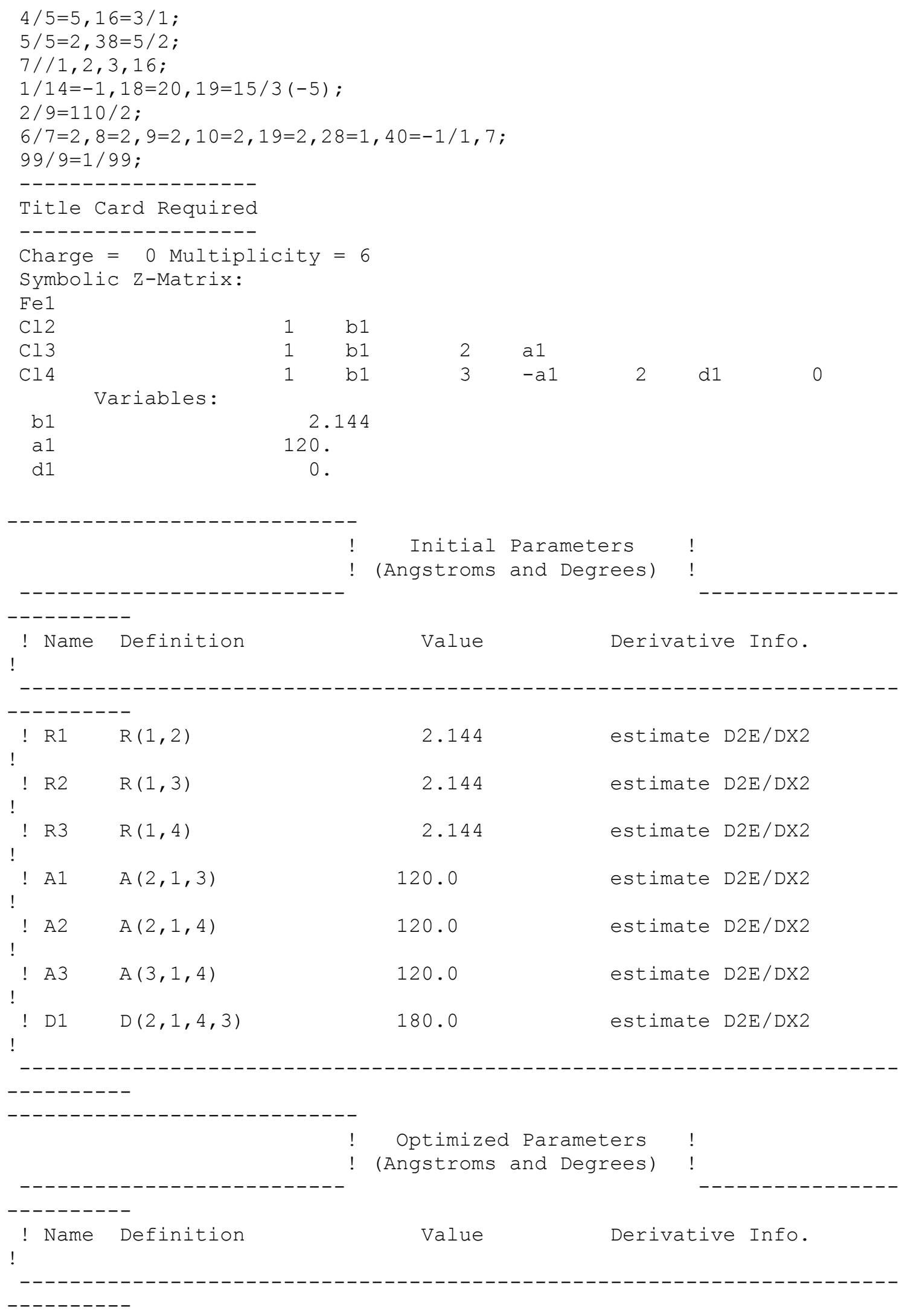




\begin{tabular}{|c|c|c|c|c|c|}
\hline $\begin{array}{ll}! & \text { R1 } \\
! & \end{array}$ & $R(1,2)$ & 2.1472 & $-\mathrm{DE} / \mathrm{DX}$ & $=$ & -0.0001 \\
\hline $\begin{array}{ll}! & \mathrm{R} 2 \\
! & \end{array}$ & $R(1,3)$ & 2.1472 & $-\mathrm{DE} / \mathrm{DX}$ & $=$ & -0.0001 \\
\hline ! R3 & $\mathrm{R}(1,4)$ & 2.1472 & -DE / DX & $=$ & -0.0001 \\
\hline $\begin{array}{ll}! & \text { A1 } \\
! & \end{array}$ & $A(2,1,3)$ & 120.0 & $-\mathrm{DE} / \mathrm{DX}$ & $=$ & 0.0 \\
\hline ! $\mathrm{A} 2$ & $A(2,1,4)$ & 120.0 & -DE / DX & $=$ & 0.0 \\
\hline ! A3 & $A(3,1,4)$ & 120.0 & -DE / DX & $=$ & 0.0 \\
\hline $\begin{array}{ll}! & \text { D1 } \\
! & \end{array}$ & $D(2,1,4,3)$ & 180.0 & $-\mathrm{DE} / \mathrm{DX}$ & $=$ & 0.0 \\
\hline
\end{tabular}

\section{$\mathrm{FeCl}_{3}$ Benzene}

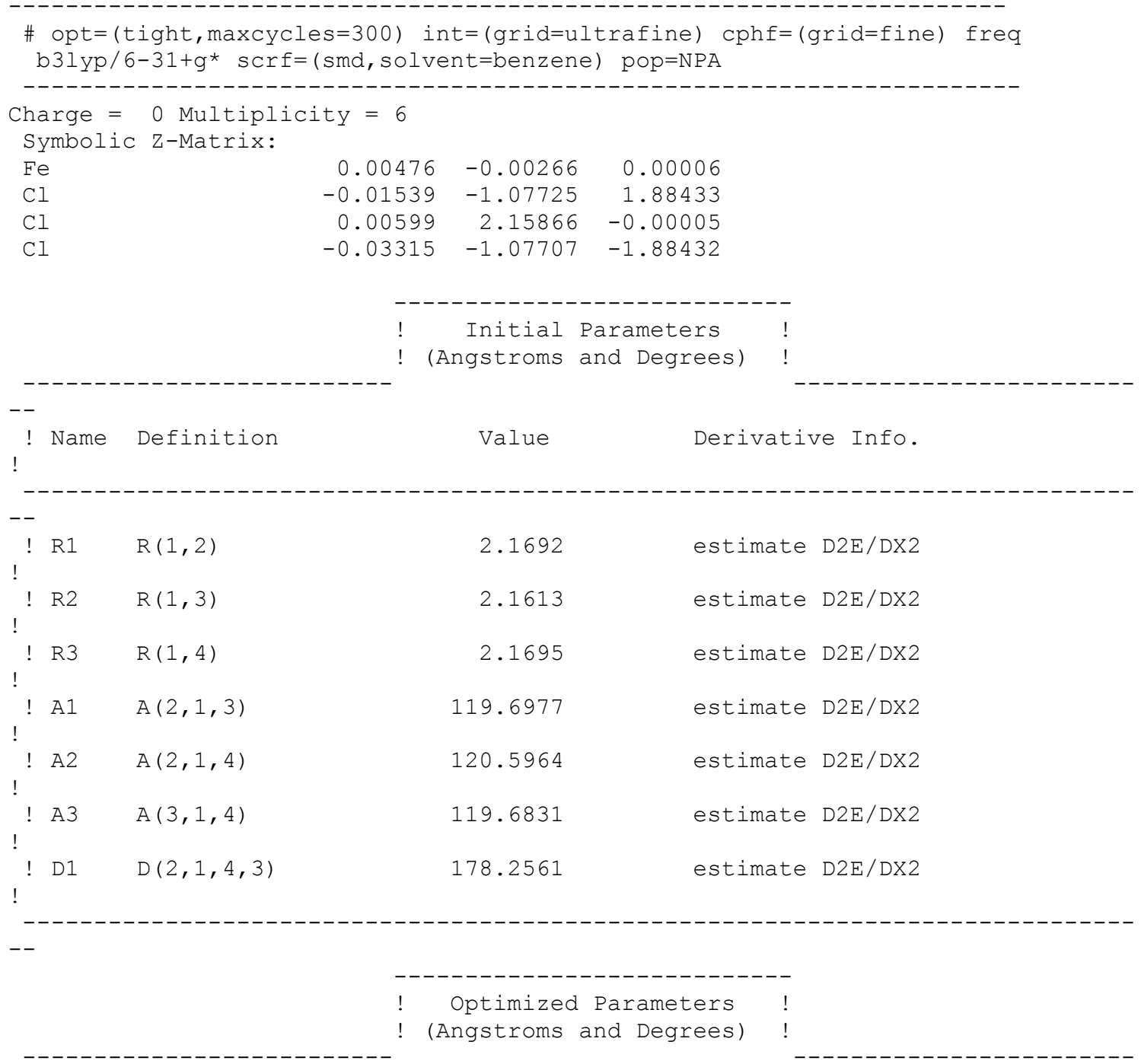

! Name Definition $\quad$ Value Derivative Info.




\begin{tabular}{|c|c|c|c|c|c|c|}
\hline-1 & & & & & & \\
\hline ! & $\mathrm{R} 1$ & $R(1,2)$ & 2.1734 & $-\mathrm{DE} / \mathrm{DX}$ & & 0.0 \\
\hline ! & $\mathrm{R} 2$ & $R(1,3)$ & 2.1706 & -DE/DX & $=$ & 0.0 \\
\hline ! & R3 & $R(1,4)$ & 2.1656 & $-\mathrm{DE} / \mathrm{DX}$ & $=$ & 0.0 \\
\hline ! & $\mathrm{A} 1$ & $A(2,1,3)$ & 119.4531 & $-\mathrm{DE} / \mathrm{DX}$ & $=$ & 0.0 \\
\hline ! & A2 & $A(2,1,4)$ & 121.3572 & $-\mathrm{DE} / \mathrm{DX}$ & $=$ & 0.0 \\
\hline ! & A3 & $A(3,1,4)$ & 119.1897 & $-\mathrm{DE} / \mathrm{DX}$ & $=$ & 0.0 \\
\hline ! & D1 & $D(2,1,4,3)$ & 180.0005 & $-\mathrm{DE} / \mathrm{DX}$ & $=$ & 0.0 \\
\hline
\end{tabular}

$--$

Summary of Natural Population Analysis:

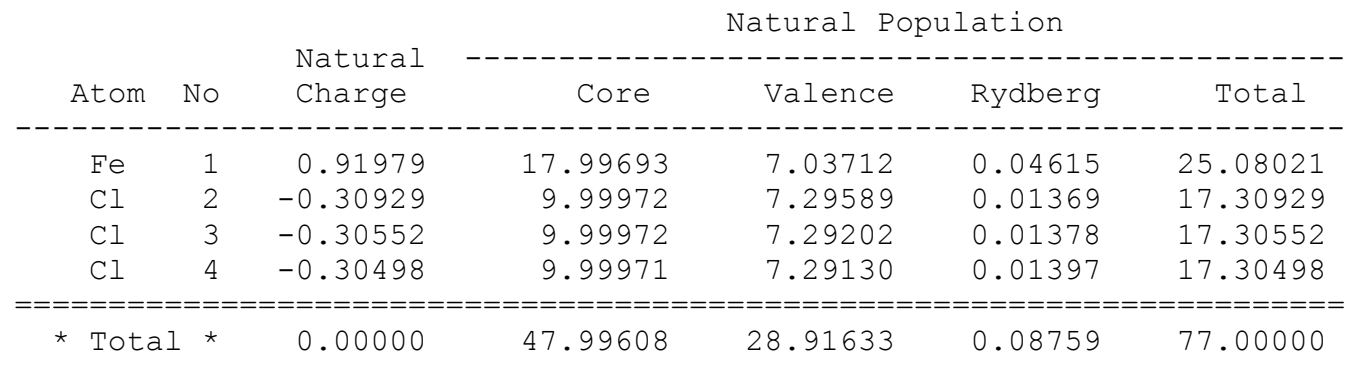

\section{Toluene carbodiimide (A), Vacuum}

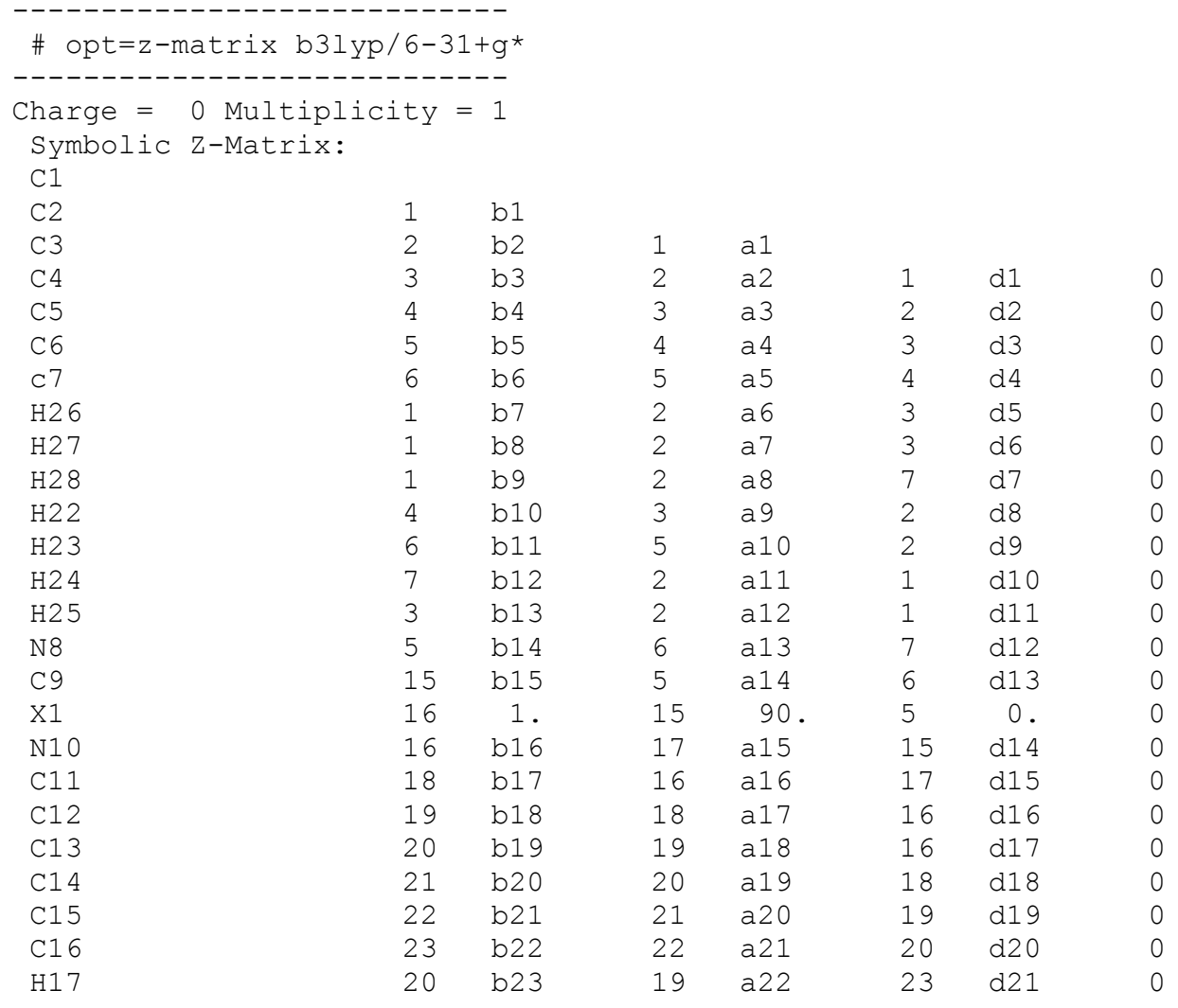




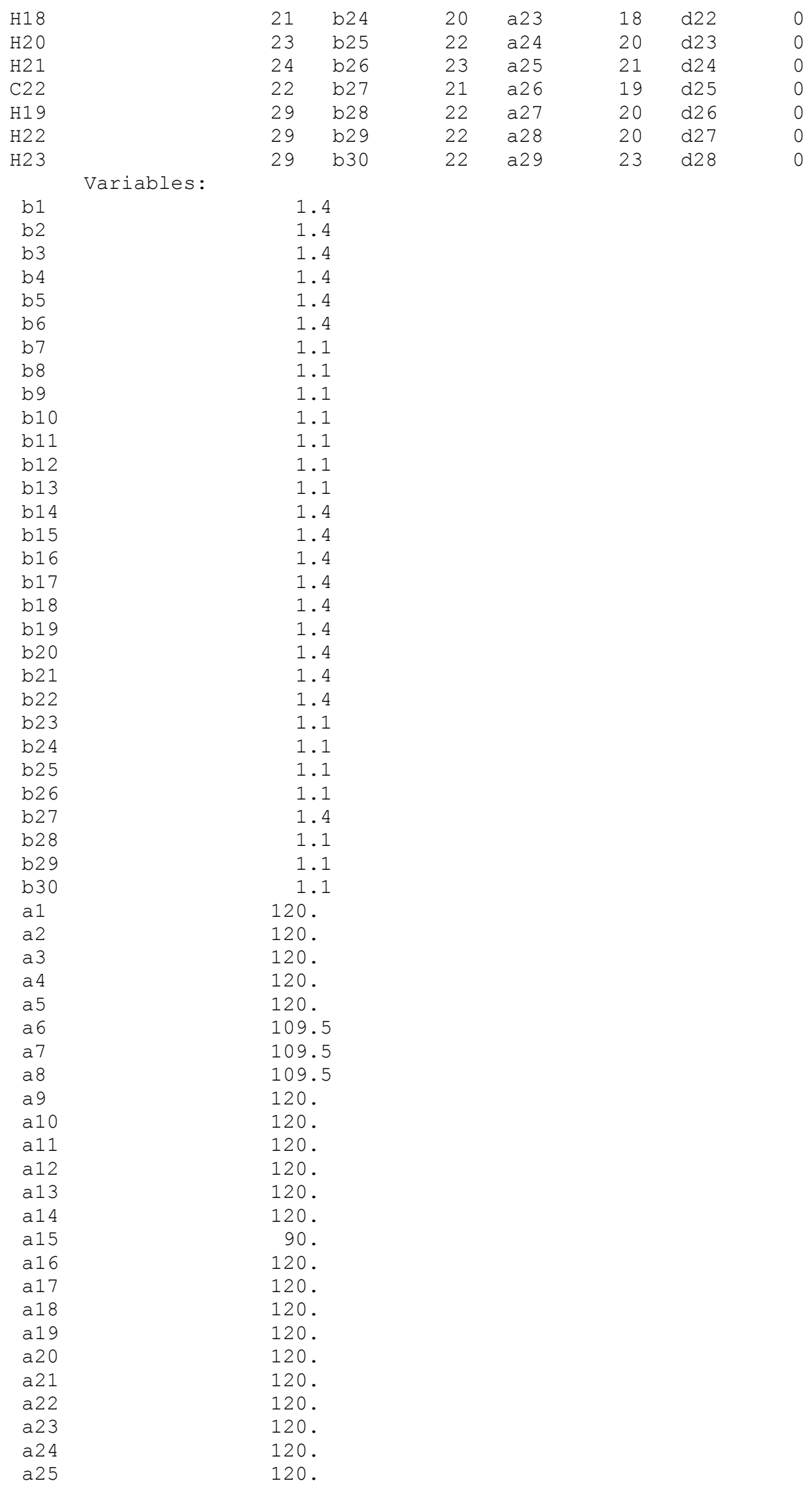




\begin{tabular}{|c|c|}
\hline a 26 & 120. \\
\hline a 27 & 109.5 \\
\hline a28 & 109.5 \\
\hline a29 & 109.5 \\
\hline d1 & 180. \\
\hline $\mathrm{d} 2$ & 0 . \\
\hline d3 & 0 . \\
\hline d4 & 0. \\
\hline d5 & -30 \\
\hline$d 6$ & 90. \\
\hline$d 7$ & 30. \\
\hline$d 8$ & 180 . \\
\hline d9 & 180 . \\
\hline d10 & 0 . \\
\hline $\mathrm{d} 11$ & 0 . \\
\hline $\mathrm{d} 12$ & 180. \\
\hline d13 & 0. \\
\hline d14 & 180 . \\
\hline d1 5 & 90. \\
\hline d16 & 180 . \\
\hline d1 7 & 180 . \\
\hline d18 & 0. \\
\hline d19 & 0 . \\
\hline $\mathrm{d} 20$ & 0 . \\
\hline $\mathrm{d} 21$ & 180 . \\
\hline $\mathrm{d} 22$ & 180 . \\
\hline d23 & 180 \\
\hline $\mathrm{d} 24$ & 180 . \\
\hline d2 5 & 180 . \\
\hline $\mathrm{d} 26$ & 90. \\
\hline d27 & -30 \\
\hline $\mathrm{d} 28$ & 30 \\
\hline
\end{tabular}

Standard orientation:

\begin{tabular}{|c|c|c|c|c|c|}
\hline \multirow{2}{*}{$\begin{array}{l}\text { Center } \\
\text { Number }\end{array}$} & \multirow{2}{*}{$\begin{array}{l}\text { Atomic } \\
\text { Number }\end{array}$} & \multirow{2}{*}{$\begin{array}{c}\text { Atomic } \\
\text { Type }\end{array}$} & \multicolumn{3}{|c|}{ Coordinates (Angstroms) } \\
\hline & & & $\mathrm{x}$ & Y & Z \\
\hline 1 & 6 & 0 & 6.146028 & -1.321397 & -0.372811 \\
\hline 2 & 6 & 0 & 4.818525 & -0.630205 & -0.160138 \\
\hline 3 & 6 & 0 & 4.640442 & 0.296514 & 0.877295 \\
\hline 4 & 6 & 0 & 3.409938 & 0.915862 & 1.096073 \\
\hline 5 & 6 & 0 & 2.317168 & 0.619593 & 0.272249 \\
\hline 6 & 6 & 0 & 2.479058 & -0.302041 & -0.774086 \\
\hline 7 & 6 & 0 & 3.715193 & -0.912380 & -0.980630 \\
\hline 8 & 1 & 0 & 6.982110 & -0.690301 & -0.051518 \\
\hline 9 & 1 & 0 & 6.203998 & -2.256741 & 0.200861 \\
\hline 10 & 1 & 0 & 6.301454 & -1.576280 & -1.427088 \\
\hline 11 & 1 & 0 & 3.282741 & 1.636076 & 1.898929 \\
\hline 12 & 1 & 0 & 1.640031 & -0.531663 & -1.426547 \\
\hline 13 & 1 & 0 & 3.823824 & -1.620876 & -1.799486 \\
\hline 14 & 1 & 0 & 5.479106 & 0.542255 & 1.525496 \\
\hline 15 & 7 & 0 & 1.100706 & 1.281241 & 0.526496 \\
\hline 16 & 6 & 0 & 0.000424 & 1.176136 & -0.003119 \\
\hline 17 & 7 & 0 & -1.099578 & 1.275972 & -0.534464 \\
\hline 18 & 6 & 0 & -2.316854 & 0.617786 & -0.275416 \\
\hline 19 & 6 & 0 & -3.407392 & 0.904917 & -1.105289 \\
\hline 20 & 6 & 0 & -4.638456 & 0.287771 & -0.883095 \\
\hline 21 & 6 & 0 & -4.819233 & -0.627472 & 0.163892 \\
\hline 22 & 6 & 0 & -3.718085 & -0.900352 & 0.990602 \\
\hline 23 & 6 & 0 & -2.481489 & -0.292233 & 0.780697 \\
\hline 24 & 1 & 0 & -3.278061 & 1.616150 & -1.915771 \\
\hline 25 & 1 & 0 & -5.475238 & 0.525900 & -1.536524 \\
\hline
\end{tabular}




$\begin{array}{rrrrrr}26 & 1 & 0 & -3.828783 & -1.600116 & 1.816673 \\ 27 & 1 & 0 & -1.644154 & -0.514609 & 1.437825 \\ 28 & 6 & 0 & -6.146402 & -1.318436 & 0.379294 \\ 29 & 1 & 0 & -6.193391 & -2.269231 & -0.169403 \\ 30 & 1 & 0 & -6.981037 & -0.699926 & 0.031053 \\ 31 & 1 & 0 & -6.313689 & -1.545497 & 1.438165 \\ -\end{array}$

(Angstroms and Degrees) !

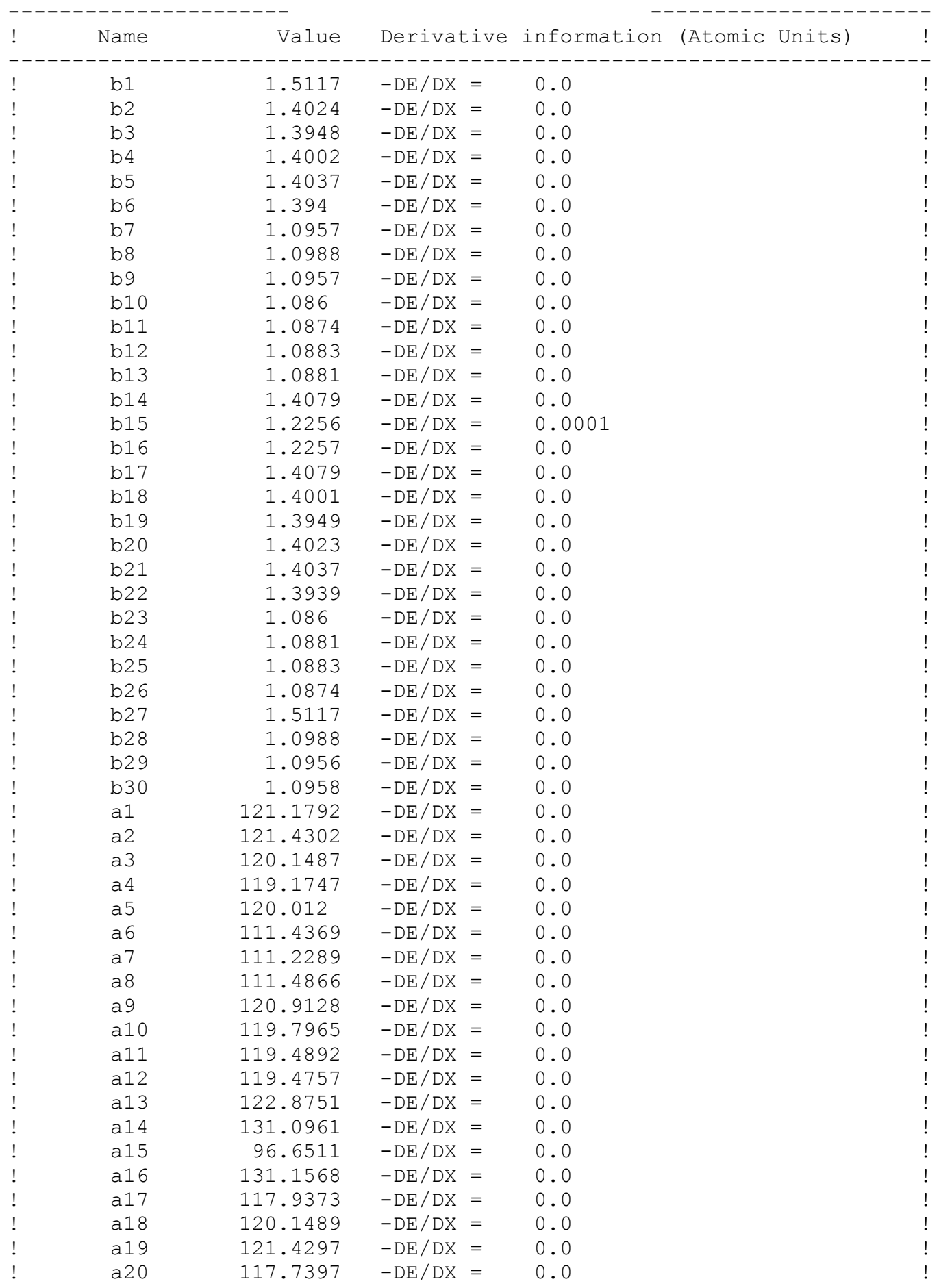




\begin{tabular}{|c|c|c|c|c|c|}
\hline ! & a21 & 121.4943 & $-\mathrm{DE} / \mathrm{DX}=$ & 0.0 & ! \\
\hline ! & a22 & 118.9385 & $-\mathrm{DE} / \mathrm{DX}=$ & 0.0 & ! \\
\hline ! & a23 & 119.0936 & $-\mathrm{DE} / \mathrm{DX}=$ & 0.0 & ! \\
\hline ! & a24 & 119.4888 & $-\mathrm{DE} / \mathrm{DX}=$ & 0.0 & ! \\
\hline ! & a2 5 & 120.1928 & $-\mathrm{DE} / \mathrm{DX}=$ & 0.0 & ! \\
\hline ! & a26 & 121.19 & $-\mathrm{DE} / \mathrm{DX}=$ & 0.0 & ! \\
\hline ! & a27 & 111.2178 & $-\mathrm{DE} / \mathrm{DX}=$ & 0.0 & ! \\
\hline ! & a28 & 111.4366 & $-\mathrm{DE} / \mathrm{DX}=$ & 0.0 & ! \\
\hline ! & a29 & 111.4958 & $-\mathrm{DE} / \mathrm{DX}=$ & 0.0 & ! \\
\hline ! & $\mathrm{d} 1$ & 181.3947 & $-\mathrm{DE} / \mathrm{DX}=$ & 0.0 & ! \\
\hline ! & $\mathrm{d} 2$ & -0.0374 & $-\mathrm{DE} / \mathrm{DX}=$ & 0.0 & ! \\
\hline ! & d3 & -0.1968 & $-\mathrm{DE} / \mathrm{DX}=$ & 0.0 & ! \\
\hline ! & $\mathrm{d} 4$ & 0.1656 & $-\mathrm{DE} / \mathrm{DX}=$ & 0.0 & ! \\
\hline ! & d5 & -29.9758 & $-\mathrm{DE} / \mathrm{DX}=$ & 0.0 & ! \\
\hline ! & $d 6$ & 89.6785 & $-\mathrm{DE} / \mathrm{DX}=$ & 0.0 & ! \\
\hline ! & $d 7$ & 30.4946 & $-\mathrm{DE} / \mathrm{DX}=$ & 0.0 & ! \\
\hline ! & d8 & 180.3106 & $-\mathrm{DE} / \mathrm{DX}=$ & 0.0 & ! \\
\hline ! & d9 & 180.432 & $-\mathrm{DE} / \mathrm{DX}=$ & 0.0 & ! \\
\hline ! & d1 0 & -1.6092 & $-\mathrm{DE} / \mathrm{DX}=$ & 0.0 & ! \\
\hline ! & d11 & 1.5865 & $-\mathrm{DE} / \mathrm{DX}=$ & 0.0 & ! \\
\hline ! & $\mathrm{d} 12$ & 179.3184 & $-\mathrm{DE} / \mathrm{DX}=$ & 0.0 & ! \\
\hline ! & d13 & 3.6024 & $-\mathrm{DE} / \mathrm{DX}=$ & -0.0001 & ! \\
\hline ! & d1 4 & 173.073 & $-\mathrm{DE} / \mathrm{DX}=$ & 0.0 & ! \\
\hline ! & d1 5 & 91.9145 & $-\mathrm{DE} / \mathrm{DX}=$ & 0.0 & ! \\
\hline ! & d1 6 & 182.888 & $-\mathrm{DE} / \mathrm{DX}=$ & 0.0 & ! \\
\hline ! & $\mathrm{d} 17$ & 178.8049 & $-\mathrm{DE} / \mathrm{DX}=$ & 0.0 & ! \\
\hline ! & d1 8 & -0.6225 & $-\mathrm{DE} / \mathrm{DX}=$ & 0.0 & ! \\
\hline ! & d19 & 0.287 & $-\mathrm{DE} / \mathrm{DX}=$ & 0.0 & ! \\
\hline ! & $\mathrm{d} 20$ & -0.1927 & $-\mathrm{DE} / \mathrm{DX}=$ & 0.0 & ! \\
\hline ! & $\mathrm{d} 21$ & 179.5526 & $-\mathrm{DE} / \mathrm{DX}=$ & 0.0 & ! \\
\hline ! & d22 & 179.2135 & $-\mathrm{DE} / \mathrm{DX}=$ & 0.0 & ! \\
\hline ! & d23 & 179.6583 & $-\mathrm{DE} / \mathrm{DX}=$ & 0.0 & ! \\
\hline ! & d2 4 & 179.4575 & $-\mathrm{DE} / \mathrm{DX}=$ & 0.0 & ! \\
\hline ! & d2 5 & 181.4859 & $-\mathrm{DE} / \mathrm{DX}=$ & 0.0 & ! \\
\hline ! & d2 6 & 89.2387 & $-\mathrm{DE} / \mathrm{DX}=$ & 0.0 & ! \\
\hline ! & d27 & -30.4264 & $-\mathrm{DE} / \mathrm{DX}=$ & 0.0 & ! \\
\hline ! & d28 & 31.6268 & $-\mathrm{DE} / \mathrm{DX}=$ & 0.0 & ! \\
\hline
\end{tabular}

\section{Toluene carbodiimide (A), Benzene}

\begin{tabular}{|c|c|c|c|}
\hline \multicolumn{4}{|c|}{$\begin{array}{l}\text { Charge }=0 \text { Multiplicity }=1 \\
\text { Symbolic } \text { Z-Matrix: }\end{array}$} \\
\hline C & 6.14603 & -1.3214 & -0.37281 \\
\hline $\mathrm{C}$ & 4.81853 & -0.63021 & -0.16014 \\
\hline $\mathrm{C}$ & 4.64044 & 0.29651 & 0.8773 \\
\hline $\mathrm{C}$ & 3.40994 & 0.91586 & 1.09607 \\
\hline C & 2.31717 & 0.61959 & 0.27225 \\
\hline $\mathrm{C}$ & 2.47906 & -0.30204 & -0.77409 \\
\hline $\mathrm{C}$ & 3.71519 & -0.91238 & -0.98063 \\
\hline $\mathrm{H}$ & 6.98211 & -0.6903 & -0.05152 \\
\hline $\mathrm{H}$ & 6.204 & -2.25674 & 0.20086 \\
\hline $\mathrm{H}$ & 6.30145 & -1.57628 & -1.42709 \\
\hline $\mathrm{H}$ & 3.28274 & 1.63608 & 1.89893 \\
\hline $\mathrm{H}$ & 1.64003 & -0.53166 & -1.42655 \\
\hline $\mathrm{H}$ & 3.82382 & -1.62088 & -1.79949 \\
\hline
\end{tabular}




$\begin{array}{llcc}\mathrm{H} & 5.47911 & 0.54226 & 1.5255 \\ \mathrm{~N} & 1.10071 & 1.28124 & 0.5265 \\ \mathrm{C} & 0.00042 & 1.17614 & -0.00312 \\ \mathrm{~N} & -1.09958 & 1.27597 & -0.53446 \\ \mathrm{C} & -2.31685 & 0.61779 & -0.27542 \\ \mathrm{C} & -3.40739 & 0.90492 & -1.10529 \\ \mathrm{C} & -4.63846 & 0.28777 & -0.8831 \\ \mathrm{C} & -4.81923 & -0.62747 & 0.16389 \\ \mathrm{C} & -3.71809 & -0.90035 & 0.9906 \\ \mathrm{C} & -2.48149 & -0.29223 & 0.7807 \\ \mathrm{H} & -3.27806 & 1.61615 & -1.91577 \\ \mathrm{H} & -5.47524 & 0.5259 & -1.53652 \\ \mathrm{H} & -3.82878 & -1.60012 & 1.81667 \\ \mathrm{H} & -1.64415 & -0.51461 & 1.43783 \\ \mathrm{C} & -6.1464 & -1.31844 & 0.37929 \\ \mathrm{H} & -6.19339 & -2.26923 & -0.1694 \\ \mathrm{H} & -6.98104 & -0.69993 & 0.03105 \\ \mathrm{H} & -6.31369 & -1.5455 & 1.43817\end{array}$

Input orientation:

\begin{tabular}{|c|c|c|c|c|c|}
\hline \multirow{2}{*}{$\begin{array}{l}\text { Center } \\
\text { Number }\end{array}$} & \multirow{2}{*}{$\begin{array}{l}\text { Atomic } \\
\text { Number }\end{array}$} & \multirow{2}{*}{$\begin{array}{c}\text { Atomic } \\
\text { Type }\end{array}$} & \multicolumn{3}{|c|}{ Coordinates (Angstroms) } \\
\hline & & & $\mathrm{x}$ & Y & Z \\
\hline 1 & 6 & 0 & 6.205137 & -1.285701 & -0.355825 \\
\hline 2 & 6 & 0 & 4.865553 & -0.616460 & -0.155463 \\
\hline 3 & 6 & 0 & 4.676106 & 0.349076 & 0.844033 \\
\hline 4 & 6 & 0 & 3.432692 & 0.947849 & 1.050790 \\
\hline 5 & 6 & 0 & 2.338421 & 0.590499 & 0.253153 \\
\hline 6 & 6 & 0 & 2.511174 & -0.370874 & -0.756094 \\
\hline 7 & 6 & 0 & 3.759004 & -0.961097 & -0.949271 \\
\hline 8 & 1 & 0 & 7.022695 & -0.670157 & 0.035658 \\
\hline 9 & 1 & 0 & 6.248159 & -2.253835 & 0.162721 \\
\hline 10 & 1 & 0 & 6.405594 & -1.478014 & -1.416346 \\
\hline 11 & 1 & 0 & 3.298185 & 1.697904 & 1.825155 \\
\hline 12 & 1 & 0 & 1.672237 & -0.648928 & -1.389318 \\
\hline 13 & 1 & 0 & 3.874704 & -1.702937 & -1.737179 \\
\hline 14 & 1 & 0 & 5.515182 & 0.642069 & 1.471641 \\
\hline 15 & 7 & 0 & 1.110394 & 1.232474 & 0.497312 \\
\hline 16 & 6 & 0 & 0.000097 & 1.123039 & -0.007600 \\
\hline 17 & 7 & 0 & -1.110181 & 1.226640 & -0.513777 \\
\hline 18 & 6 & 0 & -2.338341 & 0.587882 & -0.261964 \\
\hline 19 & 6 & 0 & -3.432499 & 0.935763 & -1.063916 \\
\hline 20 & 6 & 0 & -4.676034 & 0.339738 & -0.850014 \\
\hline 21 & 6 & 0 & -4.865697 & -0.613636 & 0.161036 \\
\hline 22 & 6 & 0 & -3.759243 & -0.948869 & 0.959006 \\
\hline 23 & 6 & 0 & -2.511309 & -0.361235 & 0.758793 \\
\hline 24 & 1 & 0 & -3.297826 & 1.676393 & -1.847271 \\
\hline 25 & 1 & 0 & -5.515025 & 0.625282 & -1.481157 \\
\hline 26 & 1 & 0 & -3.875118 & -1.681136 & 1.755795 \\
\hline 27 & 1 & 0 & -1.672446 & -0.631777 & 1.395359 \\
\hline 28 & 6 & 0 & -6.205401 & -1.280168 & 0.369461 \\
\hline 29 & 1 & 0 & -6.248021 & -2.255213 & -0.135996 \\
\hline 30 & 1 & 0 & -7.022697 & -0.669986 & -0.030854 \\
\hline 31 & 1 & 0 & -6.406594 & -1.458166 & 1.432345 \\
\hline
\end{tabular}

Standard orientation:

$\begin{array}{llll}\text { Center } & \text { Atomic } & \text { Atomic } & \text { Coordinates (Angstroms) } \\ \text { Number } & \text { Number } & \text { Type } & \mathrm{X}\end{array}$




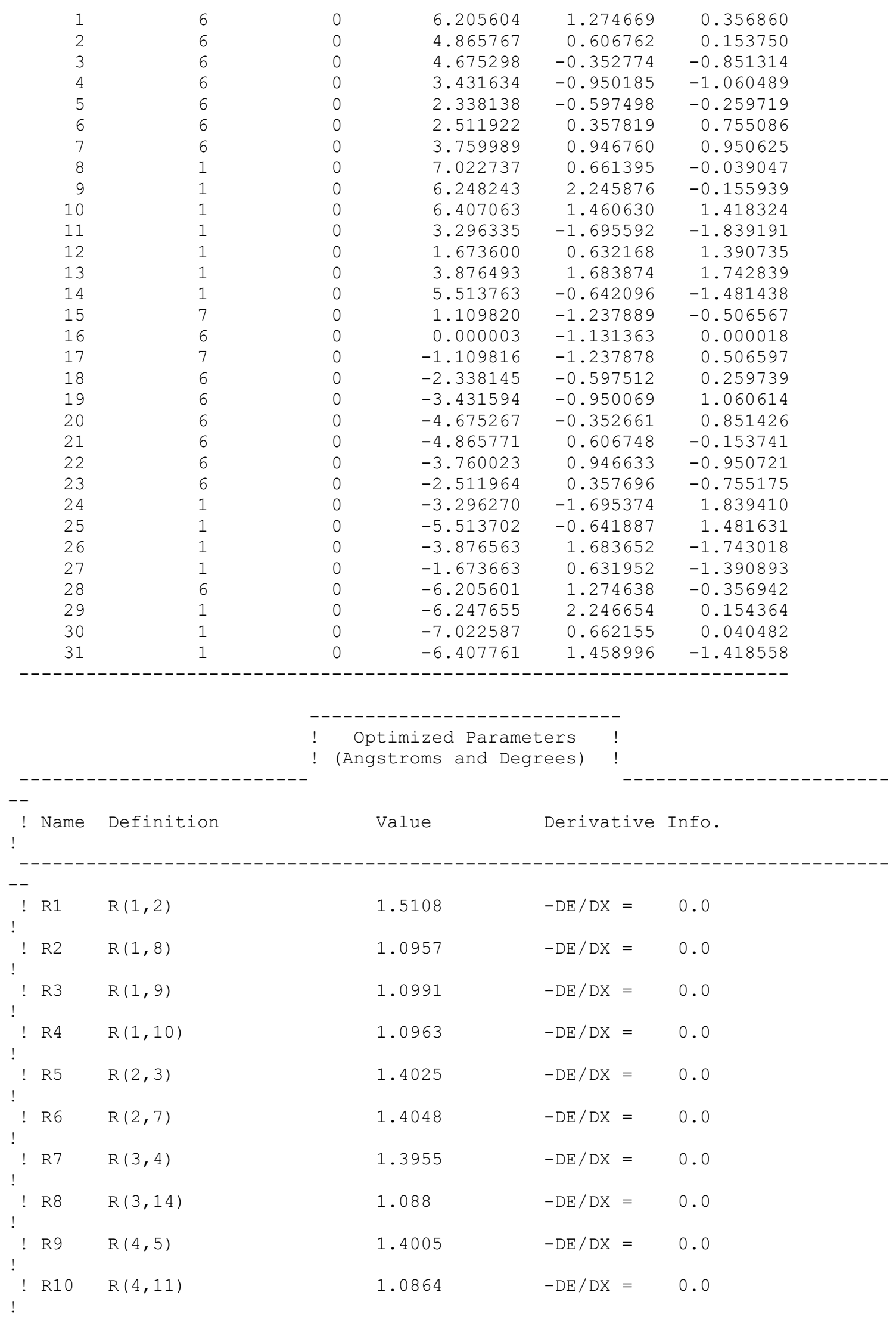




\begin{tabular}{|c|c|c|}
\hline$!$ & $\mathrm{R} 11$ & $R(5,6)$ \\
\hline ! & $\mathrm{R} 12$ & $R(5,15)$ \\
\hline ! & & \\
\hline$!$ & R13 & $R(6,7)$ \\
\hline$!$ & R14 & $R(6,12)$ \\
\hline ! & R15 & $R(7,13)$ \\
\hline ! & R16 & $R(15,16)$ \\
\hline ! & R17 & $R(16,17)$ \\
\hline ! & R18 & $R(17,18)$ \\
\hline ! & R19 & $R(18,19)$ \\
\hline ! & R20 & $R(18,23)$ \\
\hline ! & R21 & $R(19,20)$ \\
\hline $\begin{array}{l}! \\
!\end{array}$ & R22 & $R(19,24)$ \\
\hline ! & R23 & $R(20,21)$ \\
\hline ! & R24 & $R(20,25)$ \\
\hline ! & R25 & $R(21,22)$ \\
\hline ! & R26 & $R(21,28)$ \\
\hline ! & R27 & $R(22,23)$ \\
\hline ! & R28 & $R(22,26)$ \\
\hline ! & R29 & $R(23,27)$ \\
\hline ! & R30 & $R(28,29)$ \\
\hline ! & R31 & $R(28,30)$ \\
\hline ! & R32 & $R(28,31)$ \\
\hline ! & $\mathrm{A} 1$ & $A(2,1,8)$ \\
\hline ! & A2 & $A(2,1,9)$ \\
\hline ! & A3 & $A(2,1,10)$ \\
\hline ! & A4 & $A(8,1,9)$ \\
\hline ! & A5 & $A(8,1,10)$ \\
\hline ! & A 6 & $A(9,1,10)$ \\
\hline ! & A 7 & $A(1,2,3)$ \\
\hline ! & A 8 & $A(1,2,7)$ \\
\hline ! & A9 & $A(3,2,7)$ \\
\hline
\end{tabular}

\begin{tabular}{|c|c|c|c|}
\hline 1.4045 & $-D E / D X$ & $=$ & 0. \\
\hline 1.4071 & -DE/DX & $=$ & 0 . \\
\hline 1.3938 & $-\mathrm{DE} / \mathrm{DX}$ & $=$ & 0 . \\
\hline 1.0872 & $-\mathrm{DE} / \mathrm{DX}$ & $=$ & 0 . \\
\hline 1.0884 & $-\mathrm{DE} / \mathrm{DX}$ & $=$ & 0 . \\
\hline 1.2246 & $-\mathrm{DE} / \mathrm{DX}$ & $=$ & 0 . \\
\hline 1.2246 & $-\mathrm{DE} / \mathrm{DX}$ & $=$ & 0 . \\
\hline 1.4071 & $-\mathrm{DE} / \mathrm{DX}$ & $=$ & 0 . \\
\hline 1.4005 & $-\mathrm{DE} / \mathrm{DX}$ & $=$ & 0 . \\
\hline 1.4045 & $-\mathrm{DE} / \mathrm{DX}$ & $=$ & 0 . \\
\hline 1.3955 & $-\mathrm{DE} / \mathrm{DX}$ & $=$ & 0 . \\
\hline 1.0864 & $-\mathrm{DE} / \mathrm{DX}$ & $=$ & 0 . \\
\hline 1.4025 & -DE/DX & $=$ & 0 . \\
\hline 1.088 & $-\mathrm{DE} / \mathrm{DX}$ & $=$ & 0 . \\
\hline 1.4048 & $-\mathrm{DE} / \mathrm{DX}$ & $=$ & 0 . \\
\hline 1.5108 & $-\mathrm{DE} / \mathrm{DX}$ & $=$ & 0 . \\
\hline 1.3938 & $-\mathrm{DE} / \mathrm{DX}$ & $=$ & 0 . \\
\hline 1.0884 & -DE/DX & $=$ & 0 . \\
\hline 1.0872 & $-\mathrm{DE} / \mathrm{DX}$ & $=$ & 0 . \\
\hline 1.0991 & -DE/DX & $=$ & 0. \\
\hline 1.0957 & -DE/DX & $=$ & 0. \\
\hline 1.0963 & - DE/DX & $=$ & 0. \\
\hline 111.4295 & $-\mathrm{DE} / \mathrm{DX}$ & $=$ & 0. \\
\hline 111.2431 & -DE/DX & $=$ & 0 \\
\hline 111.6 & $-\mathrm{DE} / \mathrm{DX}$ & $=$ & 0. \\
\hline 107.2816 & $-\mathrm{DE} / \mathrm{DX}$ & $=$ & 0. \\
\hline 107.9234 & $-\mathrm{DE} / \mathrm{DX}$ & $=$ & 0. \\
\hline 107.1407 & $-\mathrm{DE} / \mathrm{DX}$ & $=$ & 0 . \\
\hline 121.2801 & $-\mathrm{DE} / \mathrm{DX}$ & $=$ & 0. \\
\hline 120.9858 & $-\mathrm{DE} / \mathrm{DX}$ & $=$ & 0. \\
\hline 117.7224 & $-\mathrm{DE} / \mathrm{DX}$ & $=$ & \\
\hline
\end{tabular}




\begin{tabular}{|c|c|c|}
\hline 1 & A10 & $A(2,3,4)$ \\
\hline$!$ & & \\
\hline ! & A11 & $A(2,3,14)$ \\
\hline ! & A12 & $A(4,3,14)$ \\
\hline ! & & \\
\hline ! & A13 & $A(3,4,5)$ \\
\hline$!$ & & \\
\hline ! & A14 & $A(3,4,11)$ \\
\hline$!$ & & \\
\hline ! & A15 & $A(5,4,11)$ \\
\hline ! & & \\
\hline ! & A16 & $A(4,5,6)$ \\
\hline$!$ & & \\
\hline ! & A17 & $A(4,5,15)$ \\
\hline$!$ & & \\
\hline ! & A18 & $A(6,5,15)$ \\
\hline ! & & \\
\hline ! & A19 & $A(5,6,7)$ \\
\hline ! & & \\
\hline ! & A20 & $A(5,6,12)$ \\
\hline$!$ & & \\
\hline ! & A21 & $A(7,6,12)$ \\
\hline ! & & \\
\hline ! & A22 & $A(2,7,6)$ \\
\hline ! & & \\
\hline ! & A23 & $A(2,7,13)$ \\
\hline$!$ & & \\
\hline ! & A2 4 & $A(6,7,13)$ \\
\hline ! & & \\
\hline ! & A25 & $A(5,15,16)$ \\
\hline ! & & \\
\hline ! & A2 6 & $\mathrm{~A}(16,17,18)$ \\
\hline ! & & \\
\hline ! & A 27 & $\mathrm{~A}(17,18,19)$ \\
\hline ! & & \\
\hline ! & A2 8 & $A(17,18,23)$ \\
\hline ! & & \\
\hline ! & A29 & $A(19,18,23)$ \\
\hline ! & & \\
\hline ! & A30 & $A(18,19,20)$ \\
\hline$!$ & & \\
\hline ! & A31 & $\mathrm{A}(18,19,24)$ \\
\hline ! & & \\
\hline ! & A32 & $A(20,19,24)$ \\
\hline ! & & \\
\hline ! & A33 & $A(19,20,21)$ \\
\hline$!$ & & \\
\hline ! & A34 & $A(19,20,25)$ \\
\hline ! & & \\
\hline ! & A35 & $A(21,20,25)$ \\
\hline ! & & \\
\hline ! & A36 & $A(20,21,22)$ \\
\hline ! & & \\
\hline ! & A37 & $\mathrm{A}(20,21,28)$ \\
\hline ! & & \\
\hline ! & A38 & $A(22,21,28)$ \\
\hline ! & & \\
\hline ! & A39 & $A(21,22,23)$ \\
\hline$!$ & & \\
\hline ! & A 40 & $A(21,22,26)$ \\
\hline$!$ & & \\
\hline
\end{tabular}

\begin{tabular}{|c|c|c|c|}
\hline 121.4212 & $-\mathrm{DE} / \mathrm{DX}$ & $=$ & 0.0 \\
\hline 119.4909 & $-\mathrm{DE} / \mathrm{DX}$ & $=$ & 0.0 \\
\hline 119.0877 & $-\mathrm{DE} / \mathrm{DX}$ & $=$ & 0.0 \\
\hline 120.1549 & $-\mathrm{DE} / \mathrm{DX}$ & $=$ & 0.0 \\
\hline 120.8074 & $-\mathrm{DE} / \mathrm{DX}$ & $=$ & 0.0 \\
\hline 119.0371 & $-\mathrm{DE} / \mathrm{DX}$ & $=$ & 0.0 \\
\hline 119.1967 & $-\mathrm{DE} / \mathrm{DX}$ & $=$ & 0.0 \\
\hline 117.8195 & $-\mathrm{DE} / \mathrm{DX}$ & $=$ & 0.0 \\
\hline 122.9796 & $-\mathrm{DE} / \mathrm{DX}$ & $=$ & 0.0 \\
\hline 119.9705 & $-\mathrm{DE} / \mathrm{DX}$ & $=$ & 0.0 \\
\hline 119.9107 & $-\mathrm{DE} / \mathrm{DX}$ & $=$ & 0.0 \\
\hline 120.1177 & $-\mathrm{DE} / \mathrm{DX}$ & $=$ & 0.0 \\
\hline 121.5335 & $-\mathrm{DE} / \mathrm{DX}$ & $=$ & 0.0 \\
\hline 119.5099 & $-\mathrm{DE} / \mathrm{DX}$ & $=$ & 0.0 \\
\hline 118.9562 & $-\mathrm{DE} / \mathrm{DX}$ & $=$ & 0.0 \\
\hline 132.7638 & $-\mathrm{DE} / \mathrm{DX}$ & $=$ & 0.0 \\
\hline 132.7648 & $-\mathrm{DE} / \mathrm{DX}$ & $=$ & 0.0 \\
\hline 117.8201 & $-\mathrm{DE} / \mathrm{DX}$ & $=$ & 0.0 \\
\hline 122.979 & $-\mathrm{DE} / \mathrm{DX}$ & $=$ & 0.0 \\
\hline 119.1968 & $-\mathrm{DE} / \mathrm{DX}$ & $=$ & 0.0 \\
\hline 120.1548 & $-\mathrm{DE} / \mathrm{DX}$ & $=$ & 0.0 \\
\hline 119.0374 & $-\mathrm{DE} / \mathrm{DX}$ & $=$ & 0.0 \\
\hline 120.8072 & $-\mathrm{DE} / \mathrm{DX}$ & $=$ & 0.0 \\
\hline 121.4213 & $-\mathrm{DE} / \mathrm{DX}$ & $=$ & 0.0 \\
\hline 119.0876 & $-\mathrm{DE} / \mathrm{DX}$ & $=$ & 0.0 \\
\hline 119.4909 & $-\mathrm{DE} / \mathrm{DX}$ & $=$ & 0.0 \\
\hline 117.7225 & $-\mathrm{DE} / \mathrm{DX}$ & $=$ & 0.0 \\
\hline 121.2822 & $-\mathrm{DE} / \mathrm{DX}$ & $=$ & 0.0 \\
\hline 120.9837 & $-\mathrm{DE} / \mathrm{DX}$ & $=$ & 0.0 \\
\hline 121.5333 & $-\mathrm{DE} / \mathrm{DX}$ & $=$ & 0 \\
\hline 119.5096 & $-\mathrm{DE} / \mathrm{DX}$ & $=$ & \\
\hline
\end{tabular}




\begin{tabular}{|c|c|c|}
\hline$!$ & A 41 & $A(23,22,26)$ \\
\hline$!$ & A 42 & $A(18,23,22)$ \\
\hline ! & & \\
\hline$!$ & A 43 & $A(18,23,27)$ \\
\hline$!$ & A 44 & $A(22,23,27)$ \\
\hline ! & A 45 & $\mathrm{~A}(21,28,29)$ \\
\hline$!$ & & \\
\hline$!$ & A 46 & $A(21,28,30)$ \\
\hline ! & A 47 & $A(21,28,31)$ \\
\hline ! & A 48 & $A(29,28,30)$ \\
\hline ! & A 49 & $A(29,28,31)$ \\
\hline ! & A 50 & $A(30,28,31)$ \\
\hline$!$ & A 51 & $\mathrm{~L}(15,16,17,12,-1)$ \\
\hline ! & A 52 & $L(15,16,17,12,-2)$ \\
\hline $\begin{array}{l}! \\
!\end{array}$ & D1 & $D(8,1,2,3)$ \\
\hline $\begin{array}{l}! \\
!\end{array}$ & D2 & $\mathrm{D}(8,1,2,7)$ \\
\hline ! & D3 & $D(9,1,2,3)$ \\
\hline ! & D4 & $\mathrm{D}(9,1,2,7)$ \\
\hline $\begin{array}{l}! \\
!\end{array}$ & D5 & $\mathrm{D}(10,1,2,3)$ \\
\hline $\begin{array}{l}! \\
!\end{array}$ & D6 & $\mathrm{D}(10,1,2,7)$ \\
\hline ! & D7 & $D(1,2,3,4)$ \\
\hline ! & D8 & $D(1,2,3,14)$ \\
\hline ! & D9 & $D(7,2,3,4)$ \\
\hline ! & D10 & $D(7,2,3,14)$ \\
\hline ! & D11 & $\mathrm{D}(1,2,7,6)$ \\
\hline ! & D12 & $\mathrm{D}(1,2,7,13)$ \\
\hline ! & D13 & $D(3,2,7,6)$ \\
\hline ! & D1 4 & $\mathrm{D}(3,2,7,13)$ \\
\hline ! & D15 & $D(2,3,4,5)$ \\
\hline ! & D16 & $\mathrm{D}(2,3,4,11)$ \\
\hline ! & D17 & $D(14,3,4,5)$ \\
\hline ! & D18 & $D(14,3,4,11)$ \\
\hline ! & D19 & $D(3,4,5,6)$ \\
\hline
\end{tabular}

\begin{tabular}{|c|c|c|c|}
\hline 118.9566 & $-\mathrm{DE} / \mathrm{DX}$ & $=$ & 0.0 \\
\hline 119.9705 & $-\mathrm{DE} / \mathrm{DX}$ & $=$ & 0.0 \\
\hline 119.9103 & $-\mathrm{DE} / \mathrm{DX}$ & $=$ & 0.0 \\
\hline 120.1181 & $-\mathrm{DE} / \mathrm{DX}$ & $=$ & 0.0 \\
\hline 111.2431 & $-\mathrm{DE} / \mathrm{DX}$ & $=$ & 0.0 \\
\hline 111.4292 & $-\mathrm{DE} / \mathrm{DX}$ & $=$ & 0.0 \\
\hline 111.5996 & $-\mathrm{DE} / \mathrm{DX}$ & $=$ & 0.0 \\
\hline 107.283 & $-\mathrm{DE} / \mathrm{DX}$ & $=$ & 0.0 \\
\hline 107.1401 & -DE/DX & $=$ & 0.0 \\
\hline 107.9233 & -DE/DX & $=$ & 0.0 \\
\hline 186.6689 & $-\mathrm{DE} / \mathrm{DX}$ & $=$ & 0.0 \\
\hline 187.8921 & $-\mathrm{DE} / \mathrm{DX}$ & $=$ & 0.0 \\
\hline-23.3735 & $-\mathrm{DE} / \mathrm{DX}$ & $=$ & 0.0 \\
\hline 157.8975 & $-\mathrm{DE} / \mathrm{DX}$ & $=$ & 0.0 \\
\hline 96.2944 & $-\mathrm{DE} / \mathrm{DX}$ & $=$ & 0.0 \\
\hline-82.4346 & $-\mathrm{DE} / \mathrm{DX}$ & $=$ & 0.0 \\
\hline-144.1018 & $-\mathrm{DE} / \mathrm{DX}$ & $=$ & 0.0 \\
\hline 37.1693 & $-\mathrm{DE} / \mathrm{DX}$ & $=$ & 0.0 \\
\hline-178.5808 & $-\mathrm{DE} / \mathrm{DX}$ & $=$ & 0.0 \\
\hline 1.601 & -DE/DX & $=$ & 0.0 \\
\hline 0.1883 & $-\mathrm{DE} / \mathrm{DX}$ & $=$ & 0.0 \\
\hline-179.63 & $-\mathrm{DE} / \mathrm{DX}$ & $=$ & 0.0 \\
\hline 178.6613 & $-\mathrm{DE} / \mathrm{DX}$ & $=$ & 0.0 \\
\hline-1.5757 & $-\mathrm{DE} / \mathrm{DX}$ & $=$ & 0.0 \\
\hline-0.1115 & $-\mathrm{DE} / \mathrm{DX}$ & $=$ & 0.0 \\
\hline 179.6515 & $-\mathrm{DE} / \mathrm{DX}$ & $=$ & 0.0 \\
\hline-0.0165 & $-\mathrm{DE} / \mathrm{DX}$ & $=$ & 0.0 \\
\hline-179.7317 & $-\mathrm{DE} / \mathrm{DX}$ & $=$ & 0.0 \\
\hline 179.8025 & $-\mathrm{DE} / \mathrm{DX}$ & $=$ & 0.0 \\
\hline 0.0873 & $-\mathrm{DE} / \mathrm{DX}$ & $=$ & 0.0 \\
\hline-0.2343 & $-D E / D X$ & $=$ & 0.0 \\
\hline
\end{tabular}




\begin{tabular}{|c|c|c|c|c|c|c|}
\hline$!$ & D20 & $D(3,4,5,15)$ & -179.5175 & -DE/DX & $=$ & 0.0 \\
\hline$!$ & D21 & $D(11,4,5,6)$ & 179.4859 & $-\mathrm{DE} / \mathrm{DX}$ & $=$ & 0.0 \\
\hline$!$ & D22 & $D(11,4,5,15)$ & 0.2027 & $-\mathrm{DE} / \mathrm{DX}$ & $=$ & 0.0 \\
\hline$!$ & D23 & $D(4,5,6,7)$ & 0.309 & $-\mathrm{DE} / \mathrm{DX}$ & $=$ & 0.0 \\
\hline$!$ & D2 4 & $\mathrm{D}(4,5,6,12)$ & -179.3238 & $-\mathrm{DE} / \mathrm{DX}$ & $=$ & 0.0 \\
\hline$!$ & D2 5 & $D(15,5,6,7)$ & 179.5533 & $-\mathrm{DE} / \mathrm{DX}$ & $=$ & 0.0 \\
\hline$!$ & D26 & $D(15,5,6,12)$ & -0.0796 & $-\mathrm{DE} / \mathrm{DX}$ & $=$ & 0.0 \\
\hline$!$ & D27 & $D(4,5,15,16)$ & -178.836 & $-\mathrm{DE} / \mathrm{DX}$ & $=$ & 0.0 \\
\hline$!$ & D28 & $\mathrm{D}(6,5,15,16)$ & 1.9099 & $-\mathrm{DE} / \mathrm{DX}$ & $=$ & 0.0 \\
\hline$!$ & D29 & $D(5,6,7,2)$ & -0.1364 & $-\mathrm{DE} / \mathrm{DX}$ & $=$ & 0.0 \\
\hline$!$ & D30 & $D(5,6,7,13)$ & -179.9007 & $-\mathrm{DE} / \mathrm{DX}$ & $=$ & 0.0 \\
\hline$!$ & D31 & $D(12,6,7,2)$ & 179.4958 & $-\mathrm{DE} / \mathrm{DX}$ & $=$ & 0.0 \\
\hline$!$ & D32 & $\mathrm{D}(12,6,7,13)$ & -0.2685 & -DE/DX & $=$ & 0.0 \\
\hline$!$ & D33 & $\mathrm{D}(5,15,17,18)$ & 97.8415 & $-\mathrm{DE} / \mathrm{DX}$ & $=$ & 0.0 \\
\hline$!$ & D3 4 & $\mathrm{D}(16,17,18,19)$ & -178.8286 & $-\mathrm{DE} / \mathrm{DX}$ & $=$ & 0.0 \\
\hline$!$ & D35 & $D(16,17,18,23)$ & 1.9156 & $-\mathrm{DE} / \mathrm{DX}$ & $=$ & 0.0 \\
\hline$!$ & D36 & $\mathrm{D}(17,18,19,20)$ & -179.5178 & $-\mathrm{DE} / \mathrm{DX}$ & $=$ & 0.0 \\
\hline$!$ & D37 & $D(17,18,19,24)$ & 0.2023 & $-\mathrm{DE} / \mathrm{DX}$ & $=$ & 0.0 \\
\hline$!$ & D38 & $\mathrm{D}(23,18,19,20)$ & -0.2329 & $-\mathrm{DE} / \mathrm{DX}$ & $=$ & 0.0 \\
\hline$!$ & D39 & $D(23,18,19,24)$ & 179.4871 & $-\mathrm{DE} / \mathrm{DX}$ & $=$ & 0.0 \\
\hline$!$ & D 40 & $D(17,18,23,22)$ & 179.5539 & $-\mathrm{DE} / \mathrm{DX}$ & $=$ & 0.0 \\
\hline$!$ & D4 1 & $D(17,18,23,27)$ & -0.079 & $-\mathrm{DE} / \mathrm{DX}$ & $=$ & 0.0 \\
\hline$!$ & D4 2 & $D(19,18,23,22)$ & 0.3079 & $-\mathrm{DE} / \mathrm{DX}$ & $=$ & 0.0 \\
\hline$!$ & D4 3 & $D(19,18,23,27)$ & -179.325 & $-\mathrm{DE} / \mathrm{DX}$ & $=$ & 0.0 \\
\hline$!$ & D 44 & $\mathrm{D}(18,19,20,21)$ & -0.0173 & $-\mathrm{DE} / \mathrm{DX}$ & $=$ & 0.0 \\
\hline$!$ & D4 5 & $\mathrm{D}(18,19,20,25)$ & 179.802 & $-D E / D X$ & $=$ & 0.0 \\
\hline$!$ & D4 6 & $\mathrm{D}(24,19,20,21)$ & -179.7323 & $-\mathrm{DE} / \mathrm{DX}$ & $=$ & 0.0 \\
\hline$!$ & D4 7 & $\mathrm{D}(24,19,20,25)$ & 0.087 & $-D E / D X$ & $=$ & 0.0 \\
\hline$!$ & D 48 & $D(19,20,21,22)$ & 0.1884 & $-\mathrm{DE} / \mathrm{DX}$ & $=$ & 0.0 \\
\hline$!$ & D4 9 & $D(19,20,21,28)$ & -178.5832 & $-\mathrm{DE} / \mathrm{DX}$ & $=$ & 0.0 \\
\hline$!$ & D50 & $D(25,20,21,22)$ & -179.6303 & $-D E / D X$ & $=$ & 0.0 \\
\hline
\end{tabular}




\begin{tabular}{|c|c|c|c|c|c|c|}
\hline ! & D51 & $D(25,20,21,28)$ & 1.5981 & $-\mathrm{DE} / \mathrm{DX}$ & $=$ & 0.0 \\
\hline ! & D52 & $D(20,21,22,23)$ & -0.1114 & $-\mathrm{DE} / \mathrm{DX}$ & $=$ & 0.0 \\
\hline ! & D53 & $\mathrm{D}(20,21,22,26)$ & 179.6515 & $-\mathrm{DE} / \mathrm{DX}$ & $=$ & 0.0 \\
\hline ! & D5 4 & $\mathrm{D}(28,21,22,23)$ & 178.664 & $-\mathrm{DE} / \mathrm{DX}$ & $=$ & 0.0 \\
\hline ! & D55 & $\mathrm{D}(28,21,22,26)$ & -1.573 & $-\mathrm{DE} / \mathrm{DX}$ & $=$ & 0.0 \\
\hline ! & D56 & $\mathrm{D}(20,21,28,29)$ & 96.3874 & -DE/DX & $=$ & 0.0 \\
\hline ! & D57 & $D(20,21,28,30)$ & -23.2821 & $-\mathrm{DE} / \mathrm{DX}$ & $=$ & 0.0 \\
\hline ! & D58 & $D(20,21,28,31)$ & -144.0098 & $-\mathrm{DE} / \mathrm{DX}$ & $=$ & 0.0 \\
\hline ! & D59 & $\mathrm{D}(22,21,28,29)$ & -82.3442 & -DE / DX & $=$ & 0.0 \\
\hline ! & D60 & $\mathrm{D}(22,21,28,30)$ & 157.9863 & $-D E / D X$ & $=$ & 0.0 \\
\hline ! & D61 & $\mathrm{D}(22,21,28,31)$ & 37.2586 & $-\mathrm{DE} / \mathrm{DX}$ & $=$ & 0.0 \\
\hline ! & D 62 & $D(21,22,23,18)$ & -0.136 & -DE/DX & $=$ & 0.0 \\
\hline ! & D 63 & $D(21,22,23,27)$ & 179.4961 & $-\mathrm{DE} / \mathrm{DX}$ & $=$ & 0.0 \\
\hline ! & D 64 & $\mathrm{D}(26,22,23,18)$ & -179.9002 & $-\mathrm{DE} / \mathrm{DX}$ & $=$ & 0.0 \\
\hline$!$ & D 65 & $D(26,22,23,27)$ & -0.2681 & $-\mathrm{DE} / \mathrm{DX}$ & $=$ & 0.0 \\
\hline
\end{tabular}

$--$

Summary of Natural Population Analysis:

\begin{tabular}{|c|c|c|c|c|c|c|}
\hline Atom & No & $\begin{array}{l}\text { Natural } \\
\text { Charge }\end{array}$ & Core & Valence & Rydberg & Total \\
\hline \multicolumn{7}{|l|}{--------} \\
\hline C & 1 & -0.70354 & 1.99946 & 4.69621 & 0.00786 & 6.70354 \\
\hline C & 2 & -0.04791 & 1.99908 & 4.03326 & 0.01557 & 6.04791 \\
\hline C & 3 & -0.23345 & 1.99905 & 4.21974 & 0.01465 & 6.23345 \\
\hline C & 4 & -0.23289 & 1.99905 & 4.21781 & 0.01603 & 6.23289 \\
\hline C & 5 & 0.10853 & 1.99887 & 3.87064 & 0.02196 & 5.89147 \\
\hline C & 6 & -0.24769 & 1.99904 & 4.23414 & 0.01450 & 6.24769 \\
\hline C & 7 & -0.23100 & 1.99906 & 4.21691 & 0.01503 & 6.23100 \\
\hline $\mathrm{H}$ & 8 & 0.24799 & 0.00000 & 0.75135 & 0.00066 & 0.75201 \\
\hline $\mathrm{H}$ & 9 & 0.25424 & 0.00000 & 0.74514 & 0.00062 & 0.74576 \\
\hline $\mathrm{H}$ & 10 & 0.24959 & 0.00000 & 0.74976 & 0.00065 & 0.75041 \\
\hline $\mathrm{H}$ & 11 & 0.25741 & 0.00000 & 0.74107 & 0.00152 & 0.74259 \\
\hline $\mathrm{H}$ & 12 & 0.25140 & 0.00000 & 0.74724 & 0.00137 & 0.74860 \\
\hline $\mathrm{H}$ & 13 & 0.24743 & 0.00000 & 0.75160 & 0.00097 & 0.75257 \\
\hline $\mathrm{H}$ & 14 & 0.24728 & 0.00000 & 0.75180 & 0.00092 & 0.75272 \\
\hline $\mathrm{N}$ & 15 & -0.50477 & 1.99913 & 5.48563 & 0.02000 & 7.50477 \\
\hline C & 16 & 0.67473 & 1.99917 & 3.30404 & 0.02205 & 5.32527 \\
\hline $\mathrm{N}$ & 17 & -0.50477 & 1.99913 & 5.48563 & 0.02000 & 7.50477 \\
\hline C & 18 & 0.10853 & 1.99887 & 3.87064 & 0.02196 & 5.89147 \\
\hline $\mathrm{C}$ & 19 & -0.23289 & 1.99905 & 4.21781 & 0.01603 & 6.23289 \\
\hline C & 20 & -0.23345 & 1.99905 & 4.21975 & 0.01466 & 6.23345 \\
\hline C & 21 & -0.04791 & 1.99908 & 4.03326 & 0.01557 & 6.04791 \\
\hline C & 22 & -0.23099 & 1.99906 & 4.21690 & 0.01503 & 6.23099 \\
\hline C & 23 & -0.24768 & 1.99904 & 4.23414 & 0.01450 & 6.24768 \\
\hline $\mathrm{H}$ & 24 & 0.25741 & 0.00000 & 0.74107 & 0.00152 & 0.74259 \\
\hline
\end{tabular}




\begin{tabular}{|c|c|c|c|c|c|c|}
\hline $\mathrm{H}$ & 25 & 0.24728 & 0.00000 & 0.75180 & 0.00092 & 0.75272 \\
\hline $\mathrm{H}$ & 26 & 0.24744 & 0.00000 & 0.75160 & 0.00097 & 0.75256 \\
\hline $\mathrm{H}$ & 27 & 0.25140 & 0.00000 & 0.74724 & 0.00137 & 0.74860 \\
\hline $\mathrm{C}$ & 28 & -0.70354 & 1.99946 & 4.69621 & 0.00786 & 6.70354 \\
\hline $\mathrm{H}$ & 29 & 0.25424 & 0.00000 & 0.74515 & 0.00062 & 0.74576 \\
\hline $\mathrm{H}$ & 30 & 0.24797 & 0.00000 & 0.75136 & 0.00066 & 0.75203 \\
\hline $\mathrm{H}$ & 31 & 0.24960 & 0.00000 & 0.74975 & 0.00065 & 0.75040 \\
\hline
\end{tabular}

\section{Toluene carbodiimide- $\mathrm{FeCl}_{3}$ Adduct (B), Vacuum}

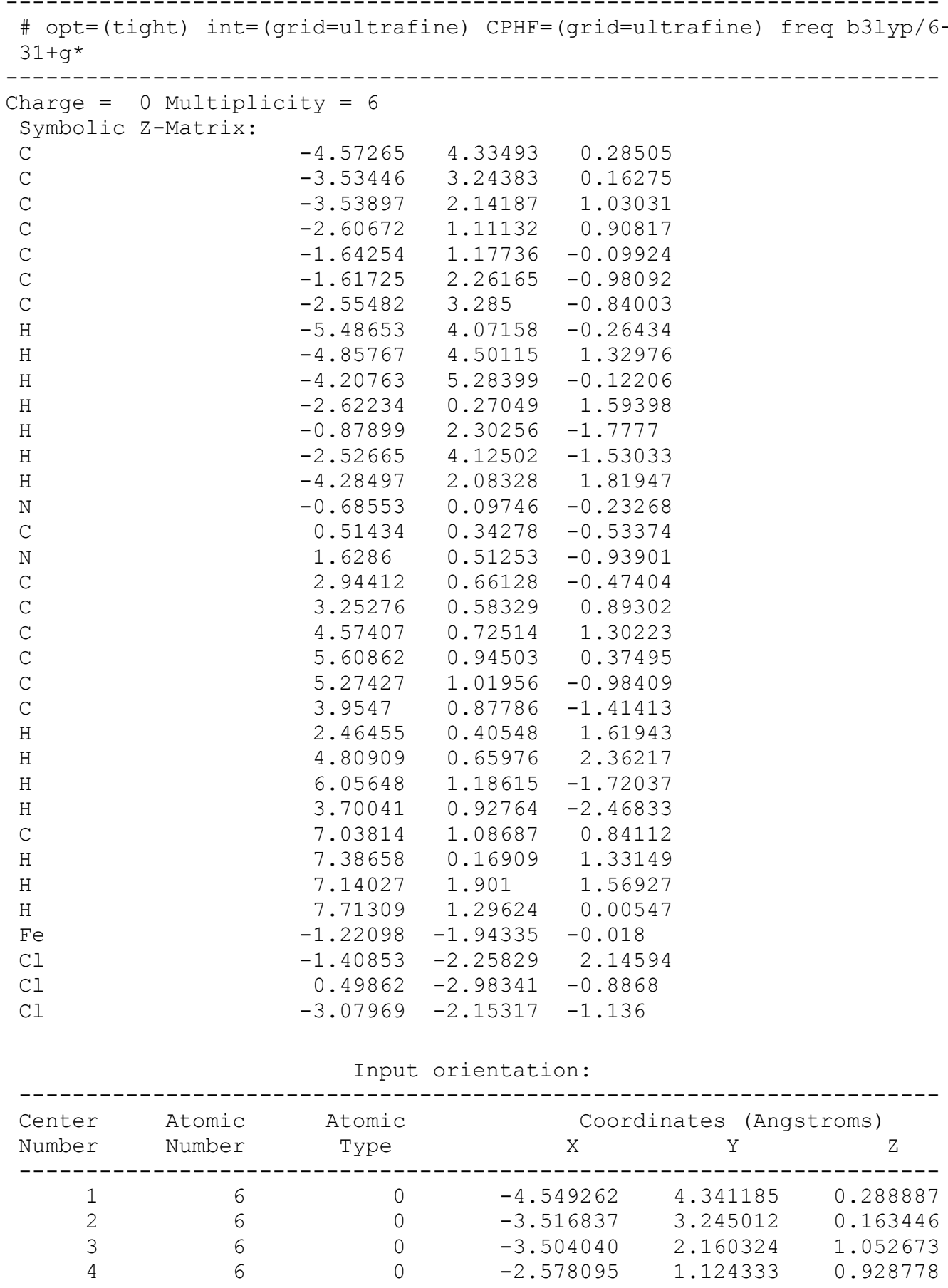




\begin{tabular}{|c|c|c|c|c|c|}
\hline 5 & 6 & 0 & -1.638168 & 1.167843 & -0.102510 \\
\hline 6 & 6 & 0 & -1.630624 & 2.233825 & -1.006100 \\
\hline 7 & 6 & 0 & -2.561473 & 3.263175 & -0.862992 \\
\hline 8 & 1 & 0 & -5.484342 & 4.059884 & -0.213932 \\
\hline 9 & 1 & 0 & -4.792527 & 4.544267 & 1.337680 \\
\hline 10 & 1 & 0 & -4.200751 & 5.274783 & -0.165494 \\
\hline 11 & 1 & 0 & -2.579689 & 0.296108 & 1.630050 \\
\hline 12 & 1 & 0 & -0.911934 & 2.255798 & -1.821275 \\
\hline 13 & 1 & 0 & -2.547573 & 4.089252 & -1.570338 \\
\hline 14 & 1 & 0 & -4.231233 & 2.119835 & 1.860336 \\
\hline 15 & 7 & 0 & -0.686947 & 0.082171 & -0.238336 \\
\hline 16 & 6 & 0 & 0.513182 & 0.324467 & -0.540058 \\
\hline 17 & 7 & 0 & 1.627395 & 0.496394 & -0.944728 \\
\hline 18 & 6 & 0 & 2.941019 & 0.650982 & -0.475662 \\
\hline 19 & 6 & 0 & 3.247494 & 0.562233 & 0.891162 \\
\hline 20 & 6 & 0 & 4.566149 & 0.715040 & 1.305177 \\
\hline 21 & 6 & 0 & 5.600275 & 0.956530 & 0.382860 \\
\hline 22 & 6 & 0 & 5.268257 & 1.040571 & -0.976201 \\
\hline 23 & 6 & 0 & 3.951459 & 0.887657 & -1.411061 \\
\hline 24 & 1 & 0 & 2.459731 & 0.368622 & 1.613992 \\
\hline 25 & 1 & 0 & 4.799184 & 0.642005 & 2.365055 \\
\hline 26 & 1 & 0 & 6.050143 & 1.224240 & -1.708778 \\
\hline 27 & 1 & 0 & 3.699135 & 0.946088 & -2.465279 \\
\hline 28 & 6 & 0 & 7.027185 & 1.108547 & 0.853715 \\
\hline 29 & 1 & 0 & 7.396680 & 0.176639 & 1.300383 \\
\hline 30 & 1 & 0 & 7.112852 & 1.889751 & 1.618886 \\
\hline 31 & 1 & 0 & 7.695843 & 1.370888 & 0.027977 \\
\hline 32 & 26 & 0 & -1.242752 & -1.952337 & -0.033500 \\
\hline 33 & 17 & 0 & -1.439357 & -2.274018 & 2.128662 \\
\hline 34 & 17 & 0 & 0.470842 & -3.003091 & -0.901391 \\
\hline 35 & 17 & 0 & -3.099446 & -2.136043 & -1.159318 \\
\hline
\end{tabular}

Standard orientation:

\begin{tabular}{|c|c|c|c|c|c|}
\hline \multirow{2}{*}{$\begin{array}{l}\text { Center } \\
\text { Number }\end{array}$} & \multirow{2}{*}{$\begin{array}{l}\text { Atomic } \\
\text { Number }\end{array}$} & \multirow{2}{*}{$\begin{array}{c}\text { Atomic } \\
\text { Type }\end{array}$} & \multicolumn{3}{|c|}{ Coordinates (Angstroms) } \\
\hline & & & $\mathrm{X}$ & $\mathrm{Y}$ & Z \\
\hline 1 & 6 & 0 & -4.513654 & 4.374362 & 0.284920 \\
\hline 2 & 6 & 0 & -3.488239 & 3.271311 & 0.162294 \\
\hline 3 & 6 & 0 & -3.480398 & 2.190446 & 1.056220 \\
\hline 4 & 6 & 0 & -2.561088 & 1.148238 & 0.935081 \\
\hline 5 & 6 & 0 & -1.622908 & 1.181467 & -0.098179 \\
\hline 6 & 6 & 0 & -1.610551 & 2.243440 & -1.006424 \\
\hline 7 & 6 & 0 & -2.534768 & 3.279119 & -0.866034 \\
\hline 8 & 1 & 0 & -5.451418 & 4.096644 & -0.214883 \\
\hline 9 & 1 & 0 & -4.753635 & 4.583502 & 1.333279 \\
\hline 10 & 1 & 0 & -4.160283 & 5.303809 & -0.174194 \\
\hline 11 & 1 & 0 & -2.566423 & 0.323099 & 1.639962 \\
\hline 12 & 1 & 0 & -0.893317 & 2.257435 & -1.823056 \\
\hline 13 & 1 & 0 & -2.517153 & 4.102009 & -1.577003 \\
\hline 14 & 1 & 0 & -4.206262 & 2.157954 & 1.865438 \\
\hline 15 & 7 & 0 & -0.678650 & 0.089372 & -0.231080 \\
\hline 16 & 6 & 0 & 0.522361 & 0.322952 & -0.536145 \\
\hline 17 & 7 & 0 & 1.636826 & 0.486247 & -0.943686 \\
\hline 18 & 6 & 0 & 2.952281 & 0.634775 & -0.477806 \\
\hline 19 & 6 & 0 & 3.260848 & 0.550088 & 0.888806 \\
\hline 20 & 6 & 0 & 4.581217 & 0.696566 & 1.299633 \\
\hline 21 & 6 & 0 & 5.615024 & 0.927663 & 0.374300 \\
\hline 22 & 6 & 0 & 5.280900 & 1.007833 & -0.984479 \\
\hline 23 & 6 & 0 & 3.962348 & 0.861146 & -1.416155 \\
\hline 24 & 1 & 0 & 2.473308 & 0.364483 & 1.613974 \\
\hline 25 & 1 & 0 & 4.815848 & 0.626710 & 2.359374 \\
\hline
\end{tabular}




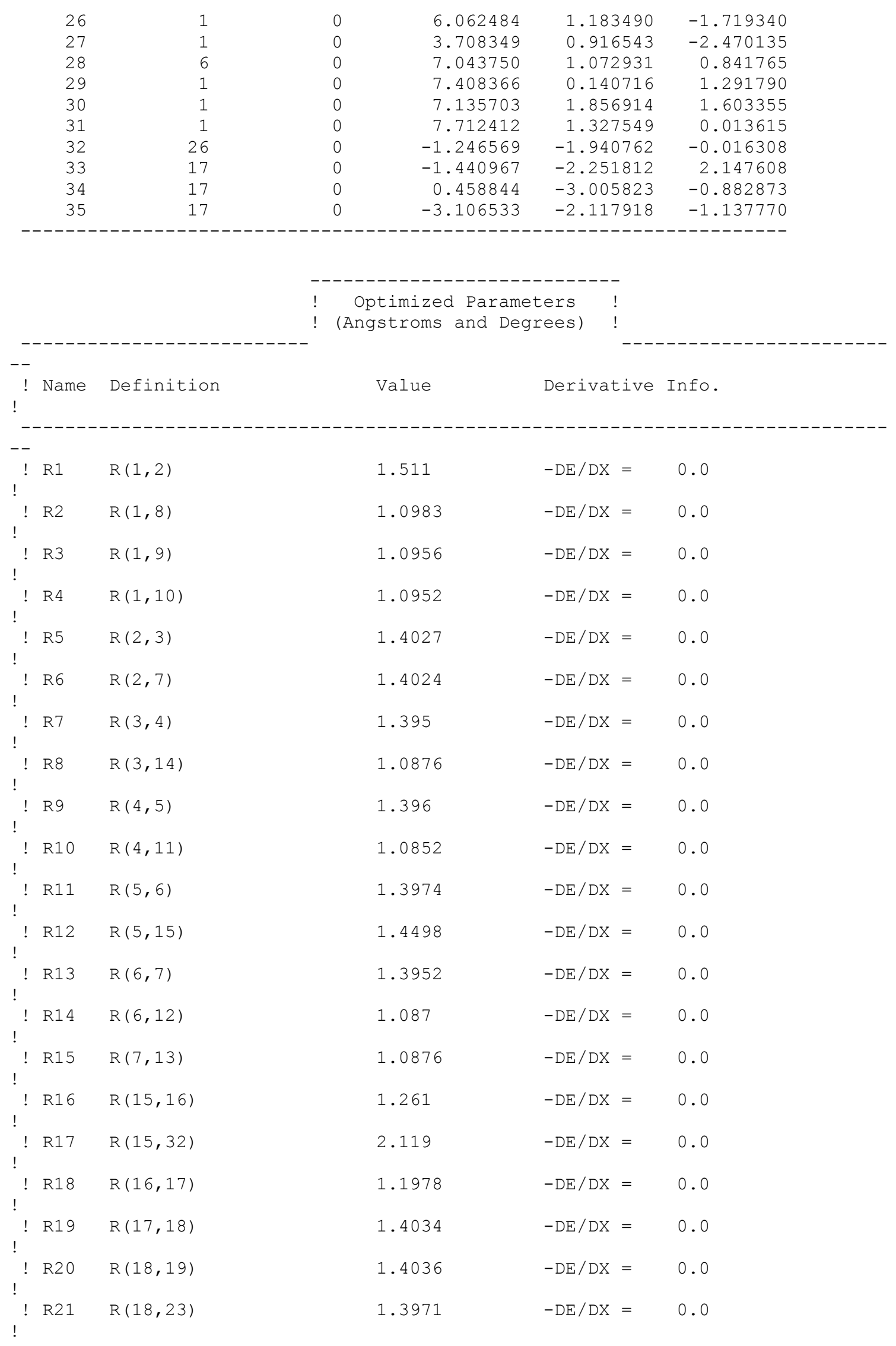




\begin{tabular}{|c|c|c|}
\hline ! & R22 & $R(19,20)$ \\
\hline$!$ & R23 & $R(19,24)$ \\
\hline ! & & \\
\hline$!$ & R24 & $R(20,21)$ \\
\hline$!$ & & \\
\hline$!$ & R25 & $R(20,25)$ \\
\hline ! & R26 & $R(21,22)$ \\
\hline$!$ & & \\
\hline$!$ & R27 & $R(21,28)$ \\
\hline$!$ & R28 & $R(22,23)$ \\
\hline ! & R29 & $R(22,26)$ \\
\hline ! & & \\
\hline ! & R30 & $R(23,27)$ \\
\hline$!$ & & \\
\hline ! & R31 & $R(28,29)$ \\
\hline$!$ & & \\
\hline$!$ & R32 & $R(28,30)$ \\
\hline ! & R33 & $R(28,31)$ \\
\hline ! & & \\
\hline ! & R34 & $R(32,33)$ \\
\hline ! & & \\
\hline ! & R35 & $R(32,34)$ \\
\hline$!$ & & \\
\hline ! & R36 & $R(32,35)$ \\
\hline$!$ & & \\
\hline ! & $\mathrm{A} 1$ & $A(2,1,8)$ \\
\hline ! & & \\
\hline ! & A2 & $A(2,1,9)$ \\
\hline ! & & \\
\hline ! & A3 & $\mathrm{A}(2,1,10)$ \\
\hline$!$ & & \\
\hline ! & A4 & $A(8,1,9)$ \\
\hline ! & & \\
\hline ! & A5 & $A(8,1,10)$ \\
\hline$!$ & & \\
\hline ! & A 6 & $A(9,1,10)$ \\
\hline ! & & \\
\hline ! & A 7 & $A(1,2,3)$ \\
\hline$!$ & & \\
\hline ! & A 8 & $A(1,2,7)$ \\
\hline ! & & \\
\hline ! & A9 & $A(3,2,7)$ \\
\hline$!$ & & \\
\hline ! & A10 & $A(2,3,4)$ \\
\hline$!$ & & \\
\hline ! & A11 & $A(2,3,14)$ \\
\hline$!$ & & \\
\hline ! & A12 & $A(4,3,14)$ \\
\hline ! & & \\
\hline ! & A13 & $A(3,4,5)$ \\
\hline$!$ & & \\
\hline ! & A14 & $A(3,4,11)$ \\
\hline ! & & \\
\hline ! & A15 & $A(5,4,11)$ \\
\hline ! & & \\
\hline ! & A16 & $A(4,5,6)$ \\
\hline 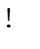 & & \\
\hline
\end{tabular}

\begin{tabular}{|c|c|c|c|}
\hline 1.3905 & $-\mathrm{DE} / \mathrm{DX}$ & $=$ & 0 . \\
\hline 1.0865 & -DE/DX & $=$ & 0 . \\
\hline 1.4066 & -DE/DX & $=$ & 0 . \\
\hline 1.0876 & -DE/DX & $=$ & 0 . \\
\hline 1.4016 & -DE/DX & $=$ & 0 . \\
\hline 1.5103 & -DE/DX & $=$ & 0 . \\
\hline 1.3951 & - DE/DX & $=$ & 0 . \\
\hline 1.0871 & -DE/DX & $=$ & 0 . \\
\hline 1.0856 & -DE/DX & $=$ & 0 . \\
\hline 1.0975 & -DE/DX & $=$ & 0 . \\
\hline 1.0969 & $-\mathrm{DE} / \mathrm{DX}$ & $=$ & 0 . \\
\hline 1.0944 & -DE/DX & $=$ & 0 . \\
\hline 2.1948 & -DE/DX & $=$ & 0 . \\
\hline 2.1895 & -DE/DX & $=$ & 0 . \\
\hline 2.1791 & $-\mathrm{DE} / \mathrm{DX}$ & $=$ & 0 . \\
\hline 110.971 & -DE/DX & $=$ & 0 . \\
\hline 111.447 & $-\mathrm{DE} / \mathrm{DX}$ & $=$ & 0 . \\
\hline 111.5015 & -DE/DX & $=$ & 0 . \\
\hline 107.2581 & $-\mathrm{DE} / \mathrm{DX}$ & $=$ & 0 . \\
\hline 107.4156 & $-\mathrm{DE} / \mathrm{DX}$ & $=$ & 0 . \\
\hline 108.0466 & $-\mathrm{DE} / \mathrm{DX}$ & $=$ & 0 . \\
\hline 120.9706 & -DE/DX & $=$ & 0 . \\
\hline 121.1203 & -DE/DX & $=$ & 0 . \\
\hline 117.8928 & $-\mathrm{DE} / \mathrm{DX}$ & $=$ & 0 . \\
\hline 121.6044 & -DE/DX & $=$ & 0 . \\
\hline 119.5707 & -DE/DX & $=$ & 0 . \\
\hline 118.8249 & $-\mathrm{DE} / \mathrm{DX}$ & $=$ & 0 . \\
\hline 119.2988 & -DE/DX & $=$ & 0 . \\
\hline 120.5577 & $-\mathrm{DE} / \mathrm{DX}$ & $=$ & 0 . \\
\hline 120.1413 & -DE/DX & $=$ & 0 . \\
\hline 120.3361 & $-\mathrm{DE} / \mathrm{DX}$ & $=$ & \\
\hline
\end{tabular}




\begin{tabular}{|c|c|c|}
\hline ! & A17 & $A(4,5,15)$ \\
\hline & A18 & $A(6,5,15)$ \\
\hline ! & A19 & $A(5,6,7)$ \\
\hline ! & A20 & $A(5,6,12)$ \\
\hline & & \\
\hline ! & A 21 & $A(7,6,12)$ \\
\hline ! & A 22 & $A(2,7,6)$ \\
\hline ! & A23 & $A(2,7,13)$ \\
\hline ! & A 24 & $A(6,7,13)$ \\
\hline ! & A 25 & $A(5,15,16)$ \\
\hline ! & A2 6 & $A(5,15,32)$ \\
\hline 1 & A 27 & $A(16,15,32)$ \\
\hline ! & A28 & $\mathrm{A}(16,17,18)$ \\
\hline ! & A29 & $\mathrm{A}(17,18,19)$ \\
\hline ! & A30 & $\mathrm{A}(17,18,23)$ \\
\hline ! & A31 & $A(19,18,23)$ \\
\hline ! & A32 & $\mathrm{A}(18,19,20)$ \\
\hline ! & A33 & $\mathrm{A}(18,19,24)$ \\
\hline ! & A3 4 & $A(20,19,24)$ \\
\hline ! & A35 & $\mathrm{A}(19,20,21)$ \\
\hline ! & A36 & $A(19,20,25)$ \\
\hline ! & A37 & $\mathrm{A}(21,20,25)$ \\
\hline ! & A38 & $\mathrm{A}(20,21,22)$ \\
\hline ! & A39 & $\mathrm{A}(20,21,28)$ \\
\hline ! & A 40 & $\mathrm{~A}(22,21,28)$ \\
\hline ! & A 41 & $A(21,22,23)$ \\
\hline ! & A 42 & $A(21,22,26)$ \\
\hline ! & A43 & $\mathrm{A}(23,22,26)$ \\
\hline ! & A 44 & $A(18,23,22)$ \\
\hline ! & A 45 & $A(18,23,27)$ \\
\hline ! & A 46 & $A(22,23,27)$ \\
\hline ! & A 47 & $A(21,28,29)$ \\
\hline
\end{tabular}

\begin{tabular}{|c|c|c|c|}
\hline 119.1836 & $-\mathrm{DE} / \mathrm{DX}$ & $=$ & 0.0 \\
\hline 120.4709 & -DE/DX & $=$ & 0.0 \\
\hline 119.5292 & $-\mathrm{DE} / \mathrm{DX}$ & $=$ & 0.0 \\
\hline 120.2589 & $-\mathrm{DE} / \mathrm{DX}$ & $=$ & 0.0 \\
\hline 120.2085 & $-\mathrm{DE} / \mathrm{DX}$ & $=$ & 0.0 \\
\hline 121.3353 & $-\mathrm{DE} / \mathrm{DX}$ & $=$ & 0.0 \\
\hline 119.6408 & $-\mathrm{DE} / \mathrm{DX}$ & $=$ & 0.0 \\
\hline 119.0212 & $-\mathrm{DE} / \mathrm{DX}$ & $=$ & 0.0 \\
\hline 120.1967 & $-\mathrm{DE} / \mathrm{DX}$ & $=$ & 0.0 \\
\hline 122.5362 & $-\mathrm{DE} / \mathrm{DX}$ & $=$ & 0.0 \\
\hline 117.2105 & $-\mathrm{DE} / \mathrm{DX}$ & $=$ & 0.0 \\
\hline 140.6769 & $-\mathrm{DE} / \mathrm{DX}$ & $=$ & 0.0 \\
\hline 121.5274 & $-\mathrm{DE} / \mathrm{DX}$ & $=$ & 0.0 \\
\hline 118.1541 & $-\mathrm{DE} / \mathrm{DX}$ & $=$ & 0.0 \\
\hline 120.3166 & $-\mathrm{DE} / \mathrm{DX}$ & $=$ & 0.0 \\
\hline 119.3444 & $-\mathrm{DE} / \mathrm{DX}$ & $=$ & 0.0 \\
\hline 120.0532 & $-\mathrm{DE} / \mathrm{DX}$ & $=$ & 0.0 \\
\hline 120.6009 & $-\mathrm{DE} / \mathrm{DX}$ & $=$ & 0.0 \\
\hline 121.3888 & $-\mathrm{DE} / \mathrm{DX}$ & $=$ & 0.0 \\
\hline 119.0737 & $-\mathrm{DE} / \mathrm{DX}$ & $=$ & 0.0 \\
\hline 119.5372 & -DE/DX & $=$ & 0.0 \\
\hline 118.1625 & - DE/DX & $=$ & 0.0 \\
\hline 120.4966 & $-\mathrm{DE} / \mathrm{DX}$ & $=$ & 0.0 \\
\hline 121.3392 & $-\mathrm{DE} / \mathrm{DX}$ & $=$ & 0.0 \\
\hline 121.2828 & $-\mathrm{DE} / \mathrm{DX}$ & $=$ & 0.0 \\
\hline 119.5516 & $-\mathrm{DE} / \mathrm{DX}$ & $=$ & 0.0 \\
\hline 119.1651 & $-\mathrm{DE} / \mathrm{DX}$ & $=$ & 0.0 \\
\hline 119.5045 & $-\mathrm{DE} / \mathrm{DX}$ & $=$ & 0.0 \\
\hline 119.4192 & $-\mathrm{DE} / \mathrm{DX}$ & $=$ & 0.0 \\
\hline 121.076 & $-\mathrm{DE} / \mathrm{DX}$ & $=$ & 0.0 \\
\hline 111.07 & $-\mathrm{DE} / \mathrm{DX}$ & $=$ & \\
\hline
\end{tabular}




\begin{tabular}{|c|c|c|}
\hline ! & A 48 & $A(21,28,30)$ \\
\hline ! & A 49 & $A(21,28,31)$ \\
\hline ! & & \\
\hline ! & A50 & $A(29,28,30)$ \\
\hline ! & A 51 & $A(29,28,31)$ \\
\hline ! & & \\
\hline ! & A52 & $A(30,28,31)$ \\
\hline$!$ & & \\
\hline$!$ & A53 & $A(15,32,33)$ \\
\hline ! & A5 4 & $A(15,32,34)$ \\
\hline ! & A55 & $A(15,32,35)$ \\
\hline$!$ & & \\
\hline$!$ & A5 6 & $A(33,32,34)$ \\
\hline$!$ & & \\
\hline$!$ & A5 7 & $A(33,32,35)$ \\
\hline ! & A58 & $A(34,32,35)$ \\
\hline ! & A5 9 & $\mathrm{~L}(15,16,17,12,-1)$ \\
\hline ! & & \\
\hline$!$ & A 60 & $L(15,16,17,12,-2)$ \\
\hline$!$ & D1 & $\mathrm{D}(8,1,2,3)$ \\
\hline$!$ & D2 & $D(8,1,2,7)$ \\
\hline$!$ & D3 & $D(9,1,2,3)$ \\
\hline ! & D4 & $D(9,1,2,7)$ \\
\hline$!$ & D5 & $\mathrm{D}(10,1,2,3)$ \\
\hline$!$ & D6 & $\mathrm{D}(10,1,2,7)$ \\
\hline$!$ & D7 & $D(1,2,3,4)$ \\
\hline$!$ & D8 & $D(1,2,3,14)$ \\
\hline$!$ & D9 & $D(7,2,3,4)$ \\
\hline$!$ & $\mathrm{D} 10$ & $D(7,2,3,14)$ \\
\hline$!$ & D11 & $D(1,2,7,6)$ \\
\hline$!$ & D12 & $D(1,2,7,13)$ \\
\hline$!$ & D13 & $D(3,2,7,6)$ \\
\hline ! & D1 4 & $\mathrm{D}(3,2,7,13)$ \\
\hline$!$ & D15 & $\mathrm{D}(2,3,4,5)$ \\
\hline$!$ & D1 6 & $D(2,3,4,11)$ \\
\hline$!$ & D17 & $D(14,3,4,5)$ \\
\hline ! & D18 & $D(14,3,4,11)$ \\
\hline
\end{tabular}

\begin{tabular}{|c|c|c|c|}
\hline 111.2828 & $-\mathrm{DE} / \mathrm{DX}$ & $=$ & 0.0 \\
\hline 111.4781 & -DE/DX & $=$ & 0.0 \\
\hline 107.1306 & -DE/DX & $=$ & 0.0 \\
\hline 107.7531 & $-\mathrm{DE} / \mathrm{DX}$ & $=$ & 0.0 \\
\hline 107.9324 & $-\mathrm{DE} / \mathrm{DX}$ & $=$ & 0.0 \\
\hline 105.0374 & -DE/DX & $=$ & 0.0 \\
\hline 102.5431 & $-\mathrm{DE} / \mathrm{DX}$ & $=$ & 0.0 \\
\hline 104.7433 & -DE/DX & $=$ & 0.0 \\
\hline 112.9715 & $-\mathrm{DE} / \mathrm{DX}$ & $=$ & 0.0 \\
\hline 114.8524 & -DE/DX & $=$ & 0.0 \\
\hline 114.9359 & $-\mathrm{DE} / \mathrm{DX}$ & $=$ & 0.0 \\
\hline 178.632 & $-\mathrm{DE} / \mathrm{DX}$ & $=$ & 0.0 \\
\hline 186.4583 & -DE/DX & $=$ & 0.0 \\
\hline-83.9608 & $-\mathrm{DE} / \mathrm{DX}$ & $=$ & 0.0 \\
\hline 94.5386 & $-\mathrm{DE} / \mathrm{DX}$ & $=$ & 0.0 \\
\hline 35.5067 & -DE/DX & $=$ & 0.0 \\
\hline-145.9939 & $-\mathrm{DE} / \mathrm{DX}$ & $=$ & 0.0 \\
\hline 156.3365 & -DE/DX & $=$ & 0.0 \\
\hline-25.164 & $-\mathrm{DE} / \mathrm{DX}$ & $=$ & 0.0 \\
\hline 178.3751 & $-\mathrm{DE} / \mathrm{DX}$ & $=$ & 0.0 \\
\hline-1.6044 & -DE/DX & $=$ & 0.0 \\
\hline-0.1714 & $-\mathrm{DE} / \mathrm{DX}$ & $=$ & 0.0 \\
\hline 179.8491 & -DE/DX & $=$ & 0.0 \\
\hline-178.1299 & $-\mathrm{DE} / \mathrm{DX}$ & $=$ & 0.0 \\
\hline 1.2716 & $-\mathrm{DE} / \mathrm{DX}$ & $=$ & 0.0 \\
\hline 0.4143 & -DE/DX & $=$ & 0.0 \\
\hline 179.8158 & $-\mathrm{DE} / \mathrm{DX}$ & $=$ & 0.0 \\
\hline 0.169 & -DE/DX & $=$ & 0.0 \\
\hline 179.6285 & -DE/DX & $=$ & 0.0 \\
\hline-179.8512 & $-\mathrm{DE} / \mathrm{DX}$ & $=$ & 0.0 \\
\hline-0.3918 & -DE/DX & $=$ & 0 \\
\hline
\end{tabular}




\begin{tabular}{|c|c|c|c|c|c|c|}
\hline$!$ & D19 & $D(3,4,5,6)$ & -0.4026 & $-\mathrm{DE} / \mathrm{DX}$ & $=$ & 0.0 \\
\hline$!$ & D20 & $D(3,4,5,15)$ & -179.2907 & $-\mathrm{DE} / \mathrm{DX}$ & $=$ & 0.0 \\
\hline$!$ & D21 & $\mathrm{D}(11,4,5,6)$ & -179.8643 & $-\mathrm{DE} / \mathrm{DX}$ & $=$ & 0.0 \\
\hline$!$ & D22 & $D(11,4,5,15)$ & 1.2475 & $-\mathrm{DE} / \mathrm{DX}$ & $=$ & 0.0 \\
\hline$!$ & D23 & $D(4,5,6,7)$ & 0.6405 & $-\mathrm{DE} / \mathrm{DX}$ & $=$ & 0.0 \\
\hline$!$ & D24 & $\mathrm{D}(4,5,6,12)$ & -178.6887 & $-\mathrm{DE} / \mathrm{DX}$ & $=$ & 0.0 \\
\hline$!$ & D25 & $D(15,5,6,7)$ & 179.5142 & $-\mathrm{DE} / \mathrm{DX}$ & $=$ & 0.0 \\
\hline$!$ & D26 & $\mathrm{D}(15,5,6,12)$ & 0.185 & $-\mathrm{DE} / \mathrm{DX}$ & $=$ & 0.0 \\
\hline$!$ & D27 & $\mathrm{D}(4,5,15,16)$ & -140.0287 & $-\mathrm{DE} / \mathrm{DX}$ & $=$ & 0.0 \\
\hline$!$ & D28 & $\mathrm{D}(4,5,15,32)$ & 42.7843 & $-\mathrm{DE} / \mathrm{DX}$ & $=$ & 0.0 \\
\hline$!$ & D29 & $\mathrm{D}(6,5,15,16)$ & 41.0847 & $-\mathrm{DE} / \mathrm{DX}$ & $=$ & 0.0 \\
\hline$!$ & D30 & $D(6,5,15,32)$ & -136.1023 & $-\mathrm{DE} / \mathrm{DX}$ & $=$ & 0.0 \\
\hline$!$ & D31 & $D(5,6,7,2)$ & -0.6519 & $-\mathrm{DE} / \mathrm{DX}$ & $=$ & 0.0 \\
\hline$!$ & D32 & $D(5,6,7,13)$ & 179.943 & $-\mathrm{DE} / \mathrm{DX}$ & $=$ & 0.0 \\
\hline$!$ & D33 & $D(12,6,7,2)$ & 178.6776 & $-\mathrm{DE} / \mathrm{DX}$ & $=$ & 0.0 \\
\hline$!$ & D34 & $D(12,6,7,13)$ & -0.7275 & $-\mathrm{DE} / \mathrm{DX}$ & $=$ & 0.0 \\
\hline$!$ & D35 & $D(5,15,17,18)$ & 99.3621 & $-\mathrm{DE} / \mathrm{DX}$ & $=$ & 0.0 \\
\hline$!$ & D36 & $\mathrm{D}(32,15,17,18)$ & -86.4753 & $-\mathrm{DE} / \mathrm{DX}$ & $=$ & 0.0 \\
\hline$!$ & D37 & $D(5,15,32,33)$ & -77.1661 & $-\mathrm{DE} / \mathrm{DX}$ & $=$ & 0.0 \\
\hline$!$ & D38 & $D(5,15,32,34)$ & 164.5551 & $-\mathrm{DE} / \mathrm{DX}$ & $=$ & 0.0 \\
\hline$!$ & D39 & $D(5,15,32,35)$ & 44.2128 & $-\mathrm{DE} / \mathrm{DX}$ & $=$ & 0.0 \\
\hline$!$ & D 40 & $D(16,15,32,33)$ & 105.5677 & $-\mathrm{DE} / \mathrm{DX}$ & $=$ & 0.0 \\
\hline$!$ & D4 1 & $\mathrm{D}(16,15,32,34)$ & -12.7111 & $-\mathrm{DE} / \mathrm{DX}$ & $=$ & 0.0 \\
\hline$!$ & D4 2 & $D(16,15,32,35)$ & -133.0534 & $-\mathrm{DE} / \mathrm{DX}$ & $=$ & 0.0 \\
\hline$!$ & D4 3 & $\mathrm{D}(16,17,18,19)$ & 2.8866 & $-\mathrm{DE} / \mathrm{DX}$ & $=$ & 0.0 \\
\hline$!$ & D 44 & $\mathrm{D}(16,17,18,23)$ & -177.6122 & $-\mathrm{DE} / \mathrm{DX}$ & $=$ & 0.0 \\
\hline$!$ & D4 5 & $\mathrm{D}(17,18,19,20)$ & 179.6273 & $-\mathrm{DE} / \mathrm{DX}$ & $=$ & 0.0 \\
\hline$!$ & D4 6 & $\mathrm{D}(17,18,19,24)$ & 0.0739 & $-\mathrm{DE} / \mathrm{DX}$ & $=$ & 0.0 \\
\hline$!$ & D 47 & $\mathrm{D}(23,18,19,20)$ & 0.1367 & $-\mathrm{DE} / \mathrm{DX}$ & $=$ & 0.0 \\
\hline$!$ & D4 8 & $\mathrm{D}(23,18,19,24)$ & -179.4166 & $-\mathrm{DE} / \mathrm{DX}$ & $=$ & 0.0 \\
\hline$!$ & D4 9 & $\mathrm{D}(17,18,23,22)$ & -179.7556 & $-\mathrm{DE} / \mathrm{DX}$ & $=$ & 0.0 \\
\hline
\end{tabular}




\begin{tabular}{|c|c|c|c|c|c|c|}
\hline$!$ & D50 & $\mathrm{D}(17,18,23,27)$ & 0.0317 & $-\mathrm{DE} / \mathrm{DX}$ & $=$ & 0.0 \\
\hline ! & & & & & & \\
\hline$!$ & D51 & $\mathrm{D}(19,18,23,22)$ & -0.2482 & -DE/DX & $=$ & 0.0 \\
\hline$!$ & D52 & $\mathrm{D}(19,18,23,27)$ & 179.5391 & $-\mathrm{DE} / \mathrm{DX}$ & $=$ & 0.0 \\
\hline$!$ & D53 & $\mathrm{D}(18,19,20,21)$ & -0.0141 & $-\mathrm{DE} / \mathrm{DX}$ & $=$ & 0.0 \\
\hline$!$ & D5 4 & $\mathrm{D}(18,19,20,25)$ & -179.7899 & $-\mathrm{DE} / \mathrm{DX}$ & $=$ & 0.0 \\
\hline$!$ & D5 5 & $\mathrm{D}(24,19,20,21)$ & 179.5368 & $-\mathrm{DE} / \mathrm{DX}$ & $=$ & 0.0 \\
\hline$!$ & D5 6 & $\mathrm{D}(24,19,20,25)$ & -0.239 & $-\mathrm{DE} / \mathrm{DX}$ & $=$ & 0.0 \\
\hline$!$ & D57 & $D(19,20,21,22)$ & 0.0044 & $-\mathrm{DE} / \mathrm{DX}$ & $=$ & 0.0 \\
\hline$!$ & D58 & $\mathrm{D}(19,20,21,28)$ & -179.5185 & $-D E / D X$ & $=$ & 0.0 \\
\hline ! & D59 & $D(25,20,21,22)$ & 179.7792 & $-\mathrm{DE} / \mathrm{DX}$ & $=$ & 0.0 \\
\hline$!$ & D60 & $\mathrm{D}(25,20,21,28)$ & 0.2563 & $-D E / D X$ & $=$ & 0.0 \\
\hline$!$ & D61 & $D(20,21,22,23)$ & -0.1187 & $-D E / D X$ & $=$ & 0.0 \\
\hline$!$ & D62 & $D(20,21,22,26)$ & -179.8594 & $-\mathrm{DE} / \mathrm{DX}$ & $=$ & 0.0 \\
\hline$!$ & D63 & $\mathrm{D}(28,21,22,23)$ & 179.4 & $-\mathrm{DE} / \mathrm{DX}$ & $=$ & 0.0 \\
\hline$!$ & D 64 & $\mathrm{D}(28,21,22,26)$ & -0.3407 & $-\mathrm{DE} / \mathrm{DX}$ & $=$ & 0.0 \\
\hline$!$ & D65 & $\mathrm{D}(20,21,28,29)$ & 64.3874 & $-\mathrm{DE} / \mathrm{DX}$ & $=$ & 0.0 \\
\hline$!$ & D66 & $\mathrm{D}(20,21,28,30)$ & -54.8766 & $-\mathrm{DE} / \mathrm{DX}$ & $=$ & 0.0 \\
\hline$!$ & D 67 & $\mathrm{D}(20,21,28,31)$ & -175.4313 & $-\mathrm{DE} / \mathrm{DX}$ & $=$ & 0.0 \\
\hline$!$ & D 68 & $\mathrm{D}(22,21,28,29)$ & -115.1201 & $-\mathrm{DE} / \mathrm{DX}$ & $=$ & 0.0 \\
\hline$!$ & D69 & $\mathrm{D}(22,21,28,30)$ & 125.6159 & $-\mathrm{DE} / \mathrm{DX}$ & $=$ & 0.0 \\
\hline$!$ & D70 & $\mathrm{D}(22,21,28,31)$ & 5.0612 & $-\mathrm{DE} / \mathrm{DX}$ & $=$ & 0.0 \\
\hline$!$ & D71 & $\mathrm{D}(21,22,23,18)$ & 0.2408 & $-\mathrm{DE} / \mathrm{DX}$ & $=$ & 0.0 \\
\hline$!$ & D72 & $\mathrm{D}(21,22,23,27)$ & -179.5429 & -DE/DX & $=$ & 0.0 \\
\hline ! & D73 & $\mathrm{D}(26,22,23,18)$ & 179.9824 & $-\mathrm{DE} / \mathrm{DX}$ & $=$ & 0.0 \\
\hline$!$ & D7 4 & $\mathrm{D}(26,22,23,27)$ & 0.1988 & $-\mathrm{DE} / \mathrm{DX}$ & $=$ & 0.0 \\
\hline
\end{tabular}

\section{Toluene carbodiimide- $\mathrm{FeCl}_{3}$ Adduct (B), Benzene}

\begin{tabular}{|c|c|c|c|c|}
\hline \multirow{2}{*}{\multicolumn{5}{|c|}{$\begin{array}{l}\text { Charge }=0 \text { Multiplicity }=6 \\
\text { Symbolic Z-Matrix: }\end{array}$}} \\
\hline & & & & \\
\hline $\mathrm{C}$ & -4.57265 & 4.33493 & 0.28505 & \\
\hline $\mathrm{C}$ & -3.53446 & 3.24383 & 0.16275 & \\
\hline
\end{tabular}




\begin{tabular}{|c|c|c|c|}
\hline $\mathrm{C}$ & -3.53897 & 2.14187 & 1.03031 \\
\hline $\mathrm{C}$ & -2.60672 & 1.11132 & 0.90817 \\
\hline $\mathrm{C}$ & -1.64254 & 1.17736 & -0.09924 \\
\hline $\mathrm{C}$ & -1.61725 & 2.26165 & -0.98092 \\
\hline C & -2.55482 & 3.285 & -0.84003 \\
\hline $\mathrm{H}$ & -5.48653 & 4.07158 & -0.26434 \\
\hline $\mathrm{H}$ & -4.85767 & 4.50115 & 1.32976 \\
\hline $\mathrm{H}$ & -4.20763 & 5.28399 & -0.12206 \\
\hline $\mathrm{H}$ & -2.62234 & 0.27049 & 1.59398 \\
\hline $\mathrm{H}$ & -0.87899 & 2.30256 & -1.7777 \\
\hline $\mathrm{H}$ & -2.52665 & 4.12502 & -1.53033 \\
\hline $\mathrm{H}$ & -4.28497 & 2.08328 & 1.81947 \\
\hline $\mathrm{N}$ & -0.68553 & 0.09746 & -0.23268 \\
\hline C & 0.51434 & 0.34278 & -0.53374 \\
\hline $\mathrm{N}$ & 1.6286 & 0.51253 & -0.93901 \\
\hline $\mathrm{C}$ & 2.94412 & 0.66128 & -0.47404 \\
\hline $\mathrm{C}$ & 3.25276 & 0.58329 & 0.89302 \\
\hline $\mathrm{C}$ & 4.57407 & 0.72514 & 1.30223 \\
\hline $\mathrm{C}$ & 5.60862 & 0.94503 & 0.37495 \\
\hline $\mathrm{C}$ & 5.27427 & 1.01956 & -0.98409 \\
\hline $\mathrm{C}$ & 3.9547 & 0.87786 & -1.41413 \\
\hline $\mathrm{H}$ & 2.46455 & 0.40548 & 1.61943 \\
\hline $\mathrm{H}$ & 4.80909 & 0.65976 & 2.36217 \\
\hline $\mathrm{H}$ & 6.05648 & 1.18615 & -1.72037 \\
\hline $\mathrm{H}$ & 3.70041 & 0.92764 & -2.46833 \\
\hline C & 7.03814 & 1.08687 & 0.84112 \\
\hline $\mathrm{H}$ & 7.38658 & 0.16909 & 1.33149 \\
\hline $\mathrm{H}$ & 7.14027 & 1.901 & 1.56927 \\
\hline $\mathrm{H}$ & 7.71309 & 1.29624 & 0.0054 \\
\hline $\mathrm{Fe}$ & -1.22098 & -1.94335 & -0.018 \\
\hline $\mathrm{Cl}$ & -1.40853 & -2.25829 & 2.1459 \\
\hline $\mathrm{Cl}$ & 0.49862 & -2.98341 & -0.8868 \\
\hline $\mathrm{Cl}$ & -3.07969 & -2.15317 & -1.136 \\
\hline
\end{tabular}

Input orientation:

\begin{tabular}{|c|c|c|c|c|c|}
\hline \multirow{2}{*}{$\begin{array}{l}\text { Center } \\
\text { Number }\end{array}$} & \multirow{2}{*}{$\begin{array}{l}\text { Atomic } \\
\text { Number }\end{array}$} & \multirow{2}{*}{$\begin{array}{c}\text { Atomic } \\
\text { Type }\end{array}$} & \multicolumn{3}{|c|}{ Coordinates (Angstroms) } \\
\hline & & & $\mathrm{X}$ & Y & Z \\
\hline & & & & & ------ \\
\hline 1 & 6 & 0 & -4.607081 & 4.335683 & 0.300575 \\
\hline 2 & 6 & 0 & -3.580522 & 3.238650 & 0.150494 \\
\hline 3 & 6 & 0 & -3.477697 & 2.206676 & 1.093702 \\
\hline 4 & 6 & 0 & -2.538284 & 1.183073 & 0.955077 \\
\hline 5 & 6 & 0 & -1.678087 & 1.187452 & -0.143593 \\
\hline 6 & 6 & 0 & -1.767288 & 2.196248 & -1.108206 \\
\hline 7 & 6 & 0 & -2.707106 & 3.214460 & -0.949151 \\
\hline 8 & 1 & 0 & -5.461980 & 4.173558 & -0.369966 \\
\hline 9 & 1 & 0 & -4.996196 & 4.381974 & 1.323257 \\
\hline 10 & 1 & 0 & -4.184939 & 5.317097 & 0.054292 \\
\hline 11 & 1 & 0 & -2.468042 & 0.396376 & 1.699778 \\
\hline 12 & 1 & 0 & -1.112524 & 2.185240 & -1.975486 \\
\hline 13 & 1 & 0 & -2.766234 & 4.000353 & -1.699165 \\
\hline 14 & 1 & 0 & -4.140549 & 2.197999 & 1.955654 \\
\hline 15 & 7 & 0 & -0.707186 & 0.116557 & -0.283086 \\
\hline 16 & 6 & 0 & 0.513040 & 0.379126 & -0.495864 \\
\hline 17 & 7 & 0 & 1.650820 & 0.542778 & -0.804041 \\
\hline 18 & 6 & 0 & 2.974302 & 0.681456 & -0.377080 \\
\hline 19 & 6 & 0 & 3.312855 & 0.614929 & 0.984101 \\
\hline 20 & 6 & 0 & 4.645746 & 0.741421 & 1.358840 \\
\hline 21 & 6 & 0 & 5.661026 & 0.934722 & 0.403843 \\
\hline 22 & 6 & 0 & 5.295270 & 1.002842 & -0.948289 \\
\hline 23 & 6 & 0 & 3.964239 & 0.876371 & -1.345389 \\
\hline 24 & 1 & 0 & 2.540918 & 0.460953 & 1.732236 \\
\hline
\end{tabular}




\begin{tabular}{|c|c|c|c|c|c|}
\hline 25 & 1 & 0 & 4.905203 & 0.683754 & 2.413479 \\
\hline 26 & 1 & 0 & 6.061831 & 1.151923 & -1.704492 \\
\hline 27 & 1 & 0 & 3.685568 & 0.921340 & -2.393858 \\
\hline 28 & 6 & 0 & 7.102529 & 1.050860 & 0.833867 \\
\hline 29 & 1 & 0 & 7.452067 & 0.118792 & 1.296838 \\
\hline 30 & 1 & 0 & 7.234367 & 1.847265 & 1.577031 \\
\hline 31 & 1 & 0 & 7.758499 & 1.268920 & -0.014825 \\
\hline 32 & 26 & 0 & -1.247571 & -1.898421 & -0.128254 \\
\hline 33 & 17 & 0 & -1.362081 & -2.308608 & 2.038386 \\
\hline 34 & 17 & 0 & 0.416122 & -2.991108 & -1.070355 \\
\hline 35 & 17 & 0 & -3.139257 & -2.157750 & -1.207584 \\
\hline
\end{tabular}

Standard orientation:

\begin{tabular}{|c|c|c|c|c|c|}
\hline \multirow{2}{*}{$\begin{array}{l}\text { Center } \\
\text { Number }\end{array}$} & \multirow{2}{*}{$\begin{array}{l}\text { Atomic } \\
\text { Number }\end{array}$} & \multirow{2}{*}{$\begin{array}{c}\text { Atomic } \\
\text { Type }\end{array}$} & \multicolumn{3}{|c|}{ Coordinates (Angstroms) } \\
\hline & & & $\mathrm{X}$ & Y & Z \\
\hline 1 & 6 & 0 & -4.597342 & 4.336102 & 0.266441 \\
\hline 2 & 6 & 0 & -3.571584 & 3.235788 & 0.136210 \\
\hline 3 & 6 & 0 & -3.451494 & 2.236118 & 1.111618 \\
\hline 4 & 6 & 0 & -2.512804 & 1.209480 & 0.991819 \\
\hline 5 & 6 & 0 & -1.670869 & 1.177985 & -0.120471 \\
\hline 6 & 6 & 0 & -1.777653 & 2.153788 & -1.116719 \\
\hline 7 & 6 & 0 & -2.716402 & 3.175722 & -0.976317 \\
\hline 8 & 1 & 0 & -5.462925 & 4.150654 & -0.384051 \\
\hline 9 & 1 & 0 & -4.969597 & 4.416212 & 1.293300 \\
\hline 10 & 1 & 0 & -4.180949 & 5.309176 & -0.019526 \\
\hline 11 & 1 & 0 & -2.428974 & 0.448260 & 1.761184 \\
\hline 12 & 1 & 0 & -1.137279 & 2.114444 & -1.993864 \\
\hline 13 & 1 & 0 & -2.789206 & 3.935973 & -1.751147 \\
\hline 14 & 1 & 0 & -4.100011 & 2.255601 & 1.984232 \\
\hline 15 & 7 & 0 & -0.700630 & 0.104087 & -0.240075 \\
\hline 16 & 6 & 0 & 0.515479 & 0.360723 & -0.481698 \\
\hline 17 & 7 & 0 & 1.647745 & 0.515210 & -0.813976 \\
\hline 18 & 6 & 0 & 2.977863 & 0.669577 & -0.413868 \\
\hline 19 & 6 & 0 & 3.338950 & 0.649078 & 0.942986 \\
\hline 20 & 6 & 0 & 4.677635 & 0.789527 & 1.291162 \\
\hline 21 & 6 & 0 & 5.676689 & 0.951833 & 0.313551 \\
\hline 22 & 6 & 0 & 5.288549 & 0.974198 & -1.033864 \\
\hline 23 & 6 & 0 & 3.951356 & 0.833023 & -1.404416 \\
\hline 24 & 1 & 0 & 2.579725 & 0.519409 & 1.708535 \\
\hline 25 & 1 & 0 & 4.954562 & 0.767522 & 2.342702 \\
\hline 26 & 1 & 0 & 6.042274 & 1.098697 & -1.807221 \\
\hline 27 & 1 & 0 & 3.655341 & 0.842522 & -2.449046 \\
\hline 28 & 6 & 0 & 7.124900 & 1.083906 & 0.715522 \\
\hline 29 & 1 & 0 & 7.483577 & 0.168264 & 1.203598 \\
\hline 30 & 1 & 0 & 7.267670 & 1.904915 & 1.429313 \\
\hline 31 & 1 & 0 & 7.766409 & 1.274125 & -0.150740 \\
\hline 32 & 26 & 0 & -1.235048 & -1.905165 & -0.008924 \\
\hline 33 & 17 & 0 & -1.313095 & -2.242636 & 2.171836 \\
\hline 34 & 17 & 0 & 0.414673 & -3.026975 & -0.941315 \\
\hline 35 & 17 & 0 & -3.143861 & -2.202604 & -1.047499 \\
\hline
\end{tabular}

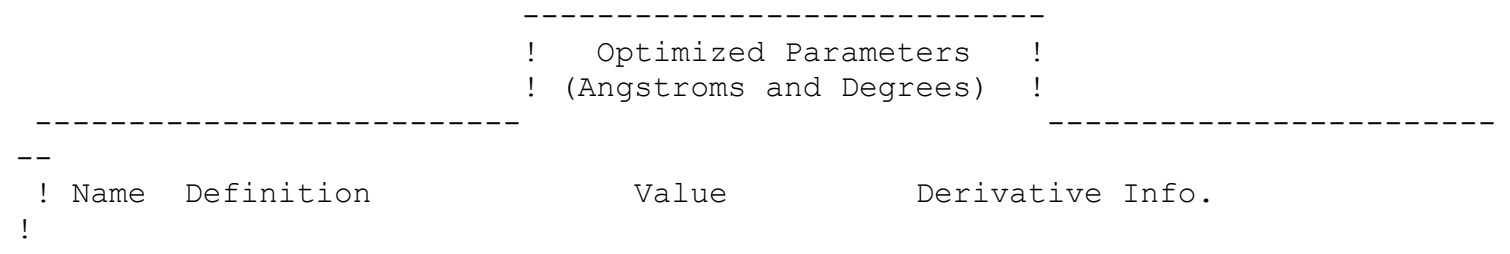




\begin{tabular}{|c|c|c|c|c|c|c|}
\hline-1 & & & & & & \\
\hline ! & R1 & $R(1,2)$ & 1.5099 & $-\mathrm{DE} / \mathrm{DX}$ & $=$ & 0.0 \\
\hline ! & R2 & $R(1,8)$ & 1.0985 & $-\mathrm{DE} / \mathrm{DX}$ & $=$ & 0.0 \\
\hline ! & R3 & $R(1,9)$ & 1.0952 & $-\mathrm{DE} / \mathrm{DX}$ & $=$ & 0.0 \\
\hline ! & R4 & $R(1,10)$ & 1.0964 & $-\mathrm{DE} / \mathrm{DX}$ & $=$ & 0.0 \\
\hline ! & R5 & $R(2,3)$ & 1.4019 & $-\mathrm{DE} / \mathrm{DX}$ & $=$ & 0.0 \\
\hline ! & R6 & $R(2,7)$ & 1.4045 & $-\mathrm{DE} / \mathrm{DX}$ & $=$ & 0.0 \\
\hline ! & R7 & $R(3,4)$ & 1.3962 & $-\mathrm{DE} / \mathrm{DX}$ & $=$ & 0.0 \\
\hline ! & R8 & $R(3,14)$ & 1.0874 & $-\mathrm{DE} / \mathrm{DX}$ & $=$ & 0.0 \\
\hline ! & R9 & $R(4,5)$ & 1.3954 & $-\mathrm{DE} / \mathrm{DX}$ & $=$ & 0.0 \\
\hline$!$ & R10 & $\mathrm{R}(4,11)$ & 1.0855 & $-\mathrm{DE} / \mathrm{DX}$ & $=$ & 0.0 \\
\hline ! & R11 & $R(5,6)$ & 1.3986 & $-\mathrm{DE} / \mathrm{DX}$ & $=$ & 0.0 \\
\hline ! & R12 & $R(5,15)$ & 1.4522 & $-\mathrm{DE} / \mathrm{DX}$ & $=$ & 0.0 \\
\hline ! & R13 & $R(6,7)$ & 1.3947 & $-\mathrm{DE} / \mathrm{DX}$ & $=$ & 0.0 \\
\hline ! & R14 & $R(6,12)$ & 1.0867 & $-\mathrm{DE} / \mathrm{DX}$ & $=$ & 0.0 \\
\hline ! & R15 & $R(7,13)$ & 1.088 & $-\mathrm{DE} / \mathrm{DX}$ & $=$ & 0.0 \\
\hline ! & R1 6 & $R(15,16)$ & 1.2662 & $-\mathrm{DE} / \mathrm{DX}$ & $=$ & 0.0 \\
\hline ! & R17 & $R(15,32)$ & 2.0919 & $-\mathrm{DE} / \mathrm{DX}$ & $=$ & 0.0 \\
\hline$!$ & R18 & $R(16,17)$ & 1.1901 & $-\mathrm{DE} / \mathrm{DX}$ & $=$ & 0.0 \\
\hline ! & R19 & $\mathrm{R}(17,18)$ & 1.3975 & $-\mathrm{DE} / \mathrm{DX}$ & $=$ & 0.0 \\
\hline ! & R20 & $\mathrm{R}(18,19)$ & 1.4042 & $-\mathrm{DE} / \mathrm{DX}$ & $=$ & 0.0 \\
\hline ! & R21 & $R(18,23)$ & 1.3984 & $-\mathrm{DE} / \mathrm{DX}$ & $=$ & 0.0 \\
\hline ! & R22 & $R(19,20)$ & 1.3903 & $-\mathrm{DE} / \mathrm{DX}$ & $=$ & 0.0 \\
\hline ! & R23 & $R(19,24)$ & 1.086 & $-\mathrm{DE} / \mathrm{DX}$ & $=$ & 0.0 \\
\hline ! & R24 & $R(20,21)$ & 1.4072 & $-\mathrm{DE} / \mathrm{DX}$ & $=$ & 0.0 \\
\hline ! & R2 5 & $R(20,25)$ & 1.0876 & $-\mathrm{DE} / \mathrm{DX}$ & $=$ & 0.0 \\
\hline ! & R26 & $R(21,22)$ & 1.4024 & $-\mathrm{DE} / \mathrm{DX}$ & $=$ & 0.0 \\
\hline ! & R27 & $R(21,28)$ & 1.5088 & $-\mathrm{DE} / \mathrm{DX}$ & $=$ & 0.0 \\
\hline ! & R28 & $\mathrm{R}(22,23)$ & 1.3947 & $-\mathrm{DE} / \mathrm{DX}$ & $=$ & 0.0 \\
\hline$!$ & R29 & $R(22,26)$ & 1.0871 & $-\mathrm{DE} / \mathrm{DX}$ & $=$ & 0.0 \\
\hline ! & R30 & $R(23,27)$ & 1.0858 & $-\mathrm{DE} / \mathrm{DX}$ & $=$ & 0.0 \\
\hline
\end{tabular}




\begin{tabular}{|c|c|c|}
\hline ! & R31 & $R(28,29)$ \\
\hline & R32 & $R(28,30)$ \\
\hline ! & & \\
\hline$!$ & R33 & $R(28,31)$ \\
\hline$!$ & R34 & $R(32,33)$ \\
\hline ! & & \\
\hline ! & R35 & $R(32,34)$ \\
\hline$!$ & R36 & $R(32,35)$ \\
\hline$!$ & $\mathrm{A} 1$ & $A(2,1,8)$ \\
\hline ! & A2 & $A(2,1,9)$ \\
\hline ! & A 3 & $\mathrm{~A}(2,1,10)$ \\
\hline ! & A 4 & $A(8,1,9)$ \\
\hline ! & A 5 & $\mathrm{~A}(8,1,10)$ \\
\hline$!$ & A 6 & $A(9,1,10)$ \\
\hline ! & A 7 & $A(1,2,3)$ \\
\hline ! & A 8 & $A(1,2,7)$ \\
\hline ! & A 9 & $A(3,2,7)$ \\
\hline ! & A10 & $A(2,3,4)$ \\
\hline ! & A11 & $A(2,3,14)$ \\
\hline ! & A12 & $A(4,3,14)$ \\
\hline ! & A13 & $A(3,4,5)$ \\
\hline ! & A14 & $A(3,4,11)$ \\
\hline$!$ & A15 & $\mathrm{A}(5,4,11)$ \\
\hline ! & A1 6 & $A(4,5,6)$ \\
\hline ! & A17 & $A(4,5,15)$ \\
\hline$!$ & A18 & $A(6,5,15)$ \\
\hline$!$ & A19 & $A(5,6,7)$ \\
\hline ! & A 20 & $A(5,6,12)$ \\
\hline ! & A 21 & $A(7,6,12)$ \\
\hline$!$ & A 22 & $A(2,7,6)$ \\
\hline$!$ & A23 & $A(2,7,13)$ \\
\hline ! & A 24 & $A(6,7,13)$ \\
\hline ! & A 25 & $A(5,15,16)$ \\
\hline
\end{tabular}

\begin{tabular}{|c|c|c|c|}
\hline 1.0978 & $-D E / D X$ & $=$ & \\
\hline 1.0972 & $-\mathrm{DE} / \mathrm{DX}$ & $=$ & \\
\hline 1.0946 & $-\mathrm{DE} / \mathrm{DX}$ & $=$ & \\
\hline 2.2081 & $-\mathrm{DE} / \mathrm{DX}$ & $=$ & \\
\hline 2.2021 & $-\mathrm{DE} / \mathrm{DX}$ & $=$ & \\
\hline 2.1933 & $-\mathrm{DE} / \mathrm{DX}$ & $=$ & 0 \\
\hline 111.1738 & $-\mathrm{DE} / \mathrm{DX}$ & $=$ & \\
\hline 111.4129 & $-\mathrm{DE} / \mathrm{DX}$ & $=$ & 0 \\
\hline 111.4856 & -DE/DX & $=$ & 0 \\
\hline 107.4413 & $-\mathrm{DE} / \mathrm{DX}$ & $=$ & 0 \\
\hline 107.1357 & $-\mathrm{DE} / \mathrm{DX}$ & $=$ & 0 \\
\hline 107.9826 & $-\mathrm{DE} / \mathrm{DX}$ & $=$ & 0 \\
\hline 121.1878 & $-\mathrm{DE} / \mathrm{DX}$ & $=$ & 0 \\
\hline 120.8724 & $-\mathrm{DE} / \mathrm{DX}$ & $=$ & \\
\hline 117.9364 & $-\mathrm{DE} / \mathrm{DX}$ & $=$ & 0 \\
\hline 121.4821 & $-\mathrm{DE} / \mathrm{DX}$ & $=$ & \\
\hline 119.637 & -DE/DX & $=$ & \\
\hline 118.8808 & $-\mathrm{DE} / \mathrm{DX}$ & $=$ & 0 \\
\hline 119.383 & $-\mathrm{DE} / \mathrm{DX}$ & $=$ & \\
\hline 120.4453 & $-\mathrm{DE} / \mathrm{DX}$ & $=$ & \\
\hline 120.1679 & $-\mathrm{DE} / \mathrm{DX}$ & $=$ & \\
\hline 120.3973 & $-\mathrm{DE} / \mathrm{DX}$ & $=$ & \\
\hline 119.0431 & $-\mathrm{DE} / \mathrm{DX}$ & $=$ & \\
\hline 120.5496 & $-\mathrm{DE} / \mathrm{DX}$ & $=$ & \\
\hline 119.3989 & $-\mathrm{DE} / \mathrm{DX}$ & $=$ & \\
\hline 120.3102 & $-\mathrm{DE} / \mathrm{DX}$ & $=$ & \\
\hline 120.2906 & $-\mathrm{DE} / \mathrm{DX}$ & $=$ & \\
\hline 121.3918 & $-\mathrm{DE} / \mathrm{DX}$ & $=$ & \\
\hline 119.571 & $-\mathrm{DE} / \mathrm{DX}$ & $=$ & \\
\hline 119.0356 & $-\mathrm{DE} / \mathrm{DX}$ & $=$ & \\
\hline 120.4995 & $-\mathrm{DE} / \mathrm{DX}$ & $=$ & \\
\hline
\end{tabular}




\begin{tabular}{|c|c|c|c|c|c|c|}
\hline$!$ & A2 6 & $A(5,15,32)$ & 122.0383 & $-\mathrm{DE} / \mathrm{DX}$ & $=$ & 0.0 \\
\hline ! & A27 & $A(16,15,32)$ & 117.4603 & $-\mathrm{DE} / \mathrm{DX}$ & $=$ & 0.0 \\
\hline$!$ & A28 & $A(16,17,18)$ & 147.1315 & $-\mathrm{DE} / \mathrm{DX}$ & $=$ & 0.0 \\
\hline$!$ & A29 & $\mathrm{A}(17,18,19)$ & 121.3161 & $-\mathrm{DE} / \mathrm{DX}$ & $=$ & 0.0 \\
\hline$!$ & A30 & $A(17,18,23)$ & 118.2077 & -DE / DX & $=$ & 0.0 \\
\hline$!$ & A31 & $A(19,18,23)$ & 120.4732 & $-\mathrm{DE} / \mathrm{DX}$ & $=$ & 0.0 \\
\hline$!$ & A32 & $A(18,19,20)$ & 119.2153 & $-\mathrm{DE} / \mathrm{DX}$ & $=$ & 0.0 \\
\hline$!$ & A33 & $A(18,19,24)$ & 120.2078 & $-\mathrm{DE} / \mathrm{DX}$ & $=$ & 0.0 \\
\hline$!$ & A 34 & $A(20,19,24)$ & 120.5761 & $-\mathrm{DE} / \mathrm{DX}$ & $=$ & 0.0 \\
\hline ! & A35 & $A(19,20,21)$ & 121.4171 & -DE / DX & $=$ & 0.0 \\
\hline ! & A36 & $A(19,20,25)$ & 119.0279 & $-\mathrm{DE} / \mathrm{DX}$ & $=$ & 0.0 \\
\hline$!$ & A 37 & $A(21,20,25)$ & 119.5541 & $-\mathrm{DE} / \mathrm{DX}$ & $=$ & 0.0 \\
\hline$!$ & A38 & $A(20,21,22)$ & 118.2186 & $-\mathrm{DE} / \mathrm{DX}$ & $=$ & 0.0 \\
\hline ! & A39 & $A(20,21,28)$ & 120.4296 & $-\mathrm{DE} / \mathrm{DX}$ & $=$ & 0.0 \\
\hline$!$ & A 40 & $A(22,21,28)$ & 121.3492 & $-\mathrm{DE} / \mathrm{DX}$ & $=$ & 0.0 \\
\hline ! & A 41 & $A(21,22,23)$ & 121.2651 & $-\mathrm{DE} / \mathrm{DX}$ & $=$ & 0.0 \\
\hline ! & A 42 & $A(21,22,26)$ & 119.5685 & $-\mathrm{DE} / \mathrm{DX}$ & $=$ & 0.0 \\
\hline ! & A 43 & $A(23,22,26)$ & 119.1657 & $-\mathrm{DE} / \mathrm{DX}$ & $=$ & 0.0 \\
\hline ! & A 44 & $A(18,23,22)$ & 119.4097 & $-\mathrm{DE} / \mathrm{DX}$ & $=$ & 0.0 \\
\hline ! & A 45 & $A(18,23,27)$ & 119.519 & $-\mathrm{DE} / \mathrm{DX}$ & $=$ & 0.0 \\
\hline ! & A4 6 & $A(22,23,27)$ & 121.0704 & -DE / DX & $=$ & 0.0 \\
\hline$!$ & A 47 & $A(21,28,29)$ & 111.041 & $-\mathrm{DE} / \mathrm{DX}$ & $=$ & 0.0 \\
\hline ! & A 48 & $A(21,28,30)$ & 111.3285 & $-\mathrm{DE} / \mathrm{DX}$ & $=$ & 0.0 \\
\hline ! & A49 & $A(21,28,31)$ & 111.5256 & $-\mathrm{DE} / \mathrm{DX}$ & $=$ & 0.0 \\
\hline ! & A50 & $A(29,28,30)$ & 106.9983 & $-\mathrm{DE} / \mathrm{DX}$ & $=$ & 0.0 \\
\hline ! & A51 & $A(29,28,31)$ & 107.7764 & $-\mathrm{DE} / \mathrm{DX}$ & $=$ & 0.0 \\
\hline ! & A5 2 & $A(30,28,31)$ & 107.9716 & $-\mathrm{DE} / \mathrm{DX}$ & $=$ & 0.0 \\
\hline ! & A53 & $A(15,32,33)$ & 105.3642 & $-\mathrm{DE} / \mathrm{DX}$ & $=$ & 0.0 \\
\hline ! & A5 4 & $A(15,32,34)$ & 104.5438 & $-\mathrm{DE} / \mathrm{DX}$ & $=$ & 0.0 \\
\hline ! & A55 & $A(15,32,35)$ & 107.4732 & $-\mathrm{DE} / \mathrm{DX}$ & $=$ & 0.0 \\
\hline$!$ & A5 6 & $A(33,32,34)$ & 111.5174 & $-\mathrm{DE} / \mathrm{DX}$ & $=$ & 0.0 \\
\hline
\end{tabular}




\begin{tabular}{|c|c|c|c|c|c|c|}
\hline ! & A5 7 & $A(33,32,35)$ & 114.5928 & $-\mathrm{DE} / \mathrm{DX}$ & $=$ & 0.0 \\
\hline$!$ & A5 8 & $A(34,32,35)$ & 112.4823 & $-\mathrm{DE} / \mathrm{DX}$ & $=$ & 0.0 \\
\hline ! & A5 9 & $\mathrm{~L}(15,16,17,12,-1)$ & 179.5567 & $-\mathrm{DE} / \mathrm{DX}$ & $=$ & 0.0 \\
\hline$!$ & A 60 & $\mathrm{~L}(15,16,17,12,-2)$ & 186.9575 & $-\mathrm{DE} / \mathrm{DX}$ & $=$ & 0.0 \\
\hline$!$ & D1 & $D(8,1,2,3)$ & -102.4581 & $-\mathrm{DE} / \mathrm{DX}$ & $=$ & 0.0 \\
\hline$!$ & D2 & $D(8,1,2,7)$ & 76.868 & $-\mathrm{DE} / \mathrm{DX}$ & $=$ & 0.0 \\
\hline$!$ & D3 & $D(9,1,2,3)$ & 17.3543 & $-\mathrm{DE} / \mathrm{DX}$ & $=$ & 0.0 \\
\hline$!$ & D4 & $D(9,1,2,7)$ & -163.3196 & $-\mathrm{DE} / \mathrm{DX}$ & $=$ & 0.0 \\
\hline$!$ & D5 & $D(10,1,2,3)$ & 138.0677 & $-\mathrm{DE} / \mathrm{DX}$ & $=$ & 0.0 \\
\hline$!$ & D6 & $\mathrm{D}(10,1,2,7)$ & -42.6062 & $-\mathrm{DE} / \mathrm{DX}$ & $=$ & 0.0 \\
\hline$!$ & D7 & $D(1,2,3,4)$ & 179.4527 & $-\mathrm{DE} / \mathrm{DX}$ & $=$ & 0.0 \\
\hline$!$ & D8 & $D(1,2,3,14)$ & -0.6306 & $-\mathrm{DE} / \mathrm{DX}$ & $=$ & 0.0 \\
\hline$!$ & D9 & $\mathrm{D}(7,2,3,4)$ & 0.1074 & $-\mathrm{DE} / \mathrm{DX}$ & $=$ & 0.0 \\
\hline$!$ & D10 & $D(7,2,3,14)$ & -179.9759 & $-\mathrm{DE} / \mathrm{DX}$ & $=$ & 0.0 \\
\hline$!$ & D11 & $D(1,2,7,6)$ & -179.0996 & $-\mathrm{DE} / \mathrm{DX}$ & $=$ & 0.0 \\
\hline$!$ & D12 & $D(1,2,7,13)$ & 0.4328 & $-\mathrm{DE} / \mathrm{DX}$ & $=$ & 0.0 \\
\hline$!$ & D13 & $D(3,2,7,6)$ & 0.2479 & $-\mathrm{DE} / \mathrm{DX}$ & $=$ & 0.0 \\
\hline$!$ & D14 & $D(3,2,7,13)$ & 179.7803 & $-\mathrm{DE} / \mathrm{DX}$ & $=$ & 0.0 \\
\hline$!$ & D15 & $D(2,3,4,5)$ & 0.2266 & $-\mathrm{DE} / \mathrm{DX}$ & $=$ & 0.0 \\
\hline$!$ & D16 & $\mathrm{D}(2,3,4,11)$ & 179.5174 & $-\mathrm{DE} / \mathrm{DX}$ & $=$ & 0.0 \\
\hline$!$ & D17 & $D(14,3,4,5)$ & -179.6907 & $-\mathrm{DE} / \mathrm{DX}$ & $=$ & 0.0 \\
\hline$!$ & D18 & $D(14,3,4,11)$ & -0.4 & $-\mathrm{DE} / \mathrm{DX}$ & $=$ & 0.0 \\
\hline$!$ & D19 & $\mathrm{D}(3,4,5,6)$ & -0.9175 & $-\mathrm{DE} / \mathrm{DX}$ & $=$ & 0.0 \\
\hline$!$ & $\mathrm{D} 20$ & $\mathrm{D}(3,4,5,15)$ & -179.7739 & $-\mathrm{DE} / \mathrm{DX}$ & $=$ & 0.0 \\
\hline$!$ & D21 & $D(11,4,5,6)$ & 179.7898 & $-\mathrm{DE} / \mathrm{DX}$ & $=$ & 0.0 \\
\hline$!$ & D22 & $D(11,4,5,15)$ & 0.9334 & $-\mathrm{DE} / \mathrm{DX}$ & $=$ & 0.0 \\
\hline$!$ & D23 & $\mathrm{D}(4,5,6,7)$ & 1.2628 & $-\mathrm{DE} / \mathrm{DX}$ & $=$ & 0.0 \\
\hline$!$ & D2 4 & $\mathrm{D}(4,5,6,12)$ & -178.5409 & $-\mathrm{DE} / \mathrm{DX}$ & $=$ & 0.0 \\
\hline ! & D25 & $\mathrm{D}(15,5,6,7)$ & -179.8981 & $-\mathrm{DE} / \mathrm{DX}$ & $=$ & 0.0 \\
\hline$!$ & D26 & $\mathrm{D}(15,5,6,12)$ & 0.2982 & $-\mathrm{DE} / \mathrm{DX}$ & $=$ & 0.0 \\
\hline$!$ & D27 & $\mathrm{D}(4,5,15,16)$ & -129.6512 & $-\mathrm{DE} / \mathrm{DX}$ & $=$ & 0.0 \\
\hline
\end{tabular}




\begin{tabular}{|c|c|c|c|c|c|c|}
\hline$!$ & D28 & $D(4,5,15,32)$ & 49.8272 & $-\mathrm{DE} / \mathrm{DX}$ & $=$ & 0.0 \\
\hline$!$ & D29 & $D(6,5,15,16)$ & 51.4942 & $-\mathrm{DE} / \mathrm{DX}$ & $=$ & 0.0 \\
\hline$!$ & D30 & $D(6,5,15,32)$ & -129.0274 & $-\mathrm{DE} / \mathrm{DX}$ & $=$ & 0.0 \\
\hline$!$ & D31 & $D(5,6,7,2)$ & -0.9291 & $-\mathrm{DE} / \mathrm{DX}$ & $=$ & 0.0 \\
\hline$!$ & D32 & $D(5,6,7,13)$ & 179.536 & $-\mathrm{DE} / \mathrm{DX}$ & $=$ & 0.0 \\
\hline$!$ & D33 & $D(12,6,7,2)$ & 178.8746 & $-\mathrm{DE} / \mathrm{DX}$ & $=$ & 0.0 \\
\hline$!$ & D34 & $D(12,6,7,13)$ & -0.6603 & $-\mathrm{DE} / \mathrm{DX}$ & $=$ & 0.0 \\
\hline$!$ & D35 & $D(5,15,17,18)$ & 99.2788 & $-\mathrm{DE} / \mathrm{DX}$ & $=$ & 0.0 \\
\hline$!$ & D36 & $\mathrm{D}(32,15,17,18)$ & -83.093 & $-\mathrm{DE} / \mathrm{DX}$ & $=$ & 0.0 \\
\hline$!$ & D37 & $\mathrm{D}(5,15,32,33)$ & -80.6719 & $-\mathrm{DE} / \mathrm{DX}$ & $=$ & 0.0 \\
\hline$!$ & D38 & $D(5,15,32,34)$ & 161.666 & $-\mathrm{DE} / \mathrm{DX}$ & $=$ & 0.0 \\
\hline$!$ & D39 & $D(5,15,32,35)$ & 41.9412 & $-\mathrm{DE} / \mathrm{DX}$ & $=$ & 0.0 \\
\hline$!$ & D4 0 & $D(16,15,32,33)$ & 98.8216 & $-\mathrm{DE} / \mathrm{DX}$ & $=$ & 0.0 \\
\hline$!$ & D4 1 & $D(16,15,32,34)$ & -18.8406 & $-\mathrm{DE} / \mathrm{DX}$ & $=$ & 0.0 \\
\hline$!$ & D42 & $D(16,15,32,35)$ & -138.5653 & $-\mathrm{DE} / \mathrm{DX}$ & $=$ & 0.0 \\
\hline$!$ & D4 3 & $\mathrm{D}(16,17,18,19)$ & 0.9277 & $-\mathrm{DE} / \mathrm{DX}$ & $=$ & 0.0 \\
\hline$!$ & D4 4 & $\mathrm{D}(16,17,18,23)$ & -179.7029 & $-\mathrm{DE} / \mathrm{DX}$ & $=$ & 0.0 \\
\hline$!$ & D 45 & $\mathrm{D}(17,18,19,20)$ & 179.1752 & $-\mathrm{DE} / \mathrm{DX}$ & $=$ & 0.0 \\
\hline$!$ & D 46 & $\mathrm{D}(17,18,19,24)$ & -0.4919 & $-\mathrm{DE} / \mathrm{DX}$ & $=$ & 0.0 \\
\hline$!$ & D 47 & $\mathrm{D}(23,18,19,20)$ & -0.18 & $-\mathrm{DE} / \mathrm{DX}$ & $=$ & 0.0 \\
\hline$!$ & D 48 & $\mathrm{D}(23,18,19,24)$ & -179.8471 & $-\mathrm{DE} / \mathrm{DX}$ & $=$ & 0.0 \\
\hline$!$ & D4 9 & $\mathrm{D}(17,18,23,22)$ & -179.2867 & -DE/DX & $=$ & 0.0 \\
\hline$!$ & D50 & $\mathrm{D}(17,18,23,27)$ & 0.3772 & $-\mathrm{DE} / \mathrm{DX}$ & $=$ & 0.0 \\
\hline$!$ & D51 & $\mathrm{D}(19,18,23,22)$ & 0.0882 & $-\mathrm{DE} / \mathrm{DX}$ & $=$ & 0.0 \\
\hline$!$ & D52 & $\mathrm{D}(19,18,23,27)$ & 179.7521 & $-\mathrm{DE} / \mathrm{DX}$ & $=$ & 0.0 \\
\hline$!$ & D53 & $\mathrm{D}(18,19,20,21)$ & 0.0135 & $-\mathrm{DE} / \mathrm{DX}$ & $=$ & 0.0 \\
\hline$!$ & D5 4 & $\mathrm{D}(18,19,20,25)$ & -179.6546 & $-\mathrm{DE} / \mathrm{DX}$ & $=$ & 0.0 \\
\hline$!$ & D55 & $\mathrm{D}(24,19,20,21)$ & 179.6794 & $-\mathrm{DE} / \mathrm{DX}$ & $=$ & 0.0 \\
\hline$!$ & D5 6 & $\mathrm{D}(24,19,20,25)$ & 0.0112 & $-\mathrm{DE} / \mathrm{DX}$ & $=$ & 0.0 \\
\hline$!$ & D57 & $\mathrm{D}(19,20,21,22)$ & 0.2382 & $-\mathrm{DE} / \mathrm{DX}$ & $=$ & 0.0 \\
\hline ! & D58 & $D(19,20,21,28)$ & -179.1832 & $-\mathrm{DE} / \mathrm{DX}$ & $=$ & 0.0 \\
\hline
\end{tabular}




\begin{tabular}{|c|c|c|c|c|c|c|}
\hline$!$ & D59 & $D(25,20,21,22)$ & 179.9046 & -DE / DX & $=$ & 0.0 \\
\hline$!$ & D60 & $D(25,20,21,28)$ & 0.4832 & $-\mathrm{DE} / \mathrm{DX}$ & $=$ & 0.0 \\
\hline ! & D61 & $\mathrm{D}(20,21,22,23)$ & -0.3325 & $-\mathrm{DE} / \mathrm{DX}$ & $=$ & 0.0 \\
\hline ! & D 62 & $D(20,21,22,26)$ & 179.9833 & $-\mathrm{DE} / \mathrm{DX}$ & $=$ & 0.0 \\
\hline ! & D63 & $\mathrm{D}(28,21,22,23)$ & 179.0833 & $-\mathrm{DE} / \mathrm{DX}$ & $=$ & 0.0 \\
\hline ! & D 64 & $\mathrm{D}(28,21,22,26)$ & -0.6009 & $-\mathrm{DE} / \mathrm{DX}$ & $=$ & 0.0 \\
\hline$!$ & D65 & $\mathrm{D}(20,21,28,29)$ & 63.8283 & $-\mathrm{DE} / \mathrm{DX}$ & $=$ & 0.0 \\
\hline$!$ & D6 6 & $D(20,21,28,30)$ & -55.28 & $-\mathrm{DE} / \mathrm{DX}$ & $=$ & 0.0 \\
\hline$!$ & D67 & $\mathrm{D}(20,21,28,31)$ & -175.9486 & $-\mathrm{DE} / \mathrm{DX}$ & $=$ & 0.0 \\
\hline$!$ & D68 & $\mathrm{D}(22,21,28,29)$ & -115.5747 & $-\mathrm{DE} / \mathrm{DX}$ & $=$ & 0.0 \\
\hline$!$ & D69 & $D(22,21,28,30)$ & 125.317 & $-\mathrm{DE} / \mathrm{DX}$ & $=$ & 0.0 \\
\hline$!$ & D70 & $\mathrm{D}(22,21,28,31)$ & 4.6485 & $-\mathrm{DE} / \mathrm{DX}$ & $=$ & 0.0 \\
\hline$!$ & D71 & $\mathrm{D}(21,22,23,18)$ & 0.1734 & $-\mathrm{DE} / \mathrm{DX}$ & $=$ & 0.0 \\
\hline$!$ & D72 & $\mathrm{D}(21,22,23,27)$ & -179.4852 & $-\mathrm{DE} / \mathrm{DX}$ & $=$ & 0.0 \\
\hline$!$ & D73 & $\mathrm{D}(26,22,23,18)$ & 179.8589 & $-\mathrm{DE} / \mathrm{DX}$ & $=$ & 0.0 \\
\hline$!$ & D74 & $\mathrm{D}(26,22,23,27)$ & 0.2003 & $-\mathrm{DE} / \mathrm{DX}$ & $=$ & .0 \\
\hline
\end{tabular}

Summary of Natural Population Analysis:

\begin{tabular}{|c|c|c|c|c|c|c|}
\hline Atom & No & $\begin{array}{l}\text { Natural } \\
\text { Charge }\end{array}$ & Core & Valence & Rydberg & Total \\
\hline C & 1 & -0.70565 & 1.99946 & 4.69825 & 0.00794 & 6.70565 \\
\hline $\mathrm{C}$ & 2 & -0.03312 & 1.99908 & 4.01884 & 0.01519 & 6.03312 \\
\hline C & 3 & -0.21995 & 1.99905 & 4.20644 & 0.01445 & 6.21995 \\
\hline C & 4 & -0.23560 & 1.99902 & 4.22055 & 0.01603 & 6.23560 \\
\hline C & 5 & 0.11702 & 1.99885 & 3.86181 & 0.02231 & 5.88298 \\
\hline C & 6 & -0.24115 & 1.99901 & 4.22790 & 0.01424 & 6.24115 \\
\hline C & 7 & -0.22254 & 1.99905 & 4.20904 & 0.01445 & 6.22254 \\
\hline $\mathrm{H}$ & 8 & 0.25897 & 0.00000 & 0.74044 & 0.00059 & 0.74103 \\
\hline $\mathrm{H}$ & 9 & 0.25002 & 0.00000 & 0.74935 & 0.00063 & 0.74998 \\
\hline $\mathrm{H}$ & 10 & 0.25208 & 0.00000 & 0.74729 & 0.00063 & 0.74792 \\
\hline $\mathrm{H}$ & 11 & 0.26424 & 0.00000 & 0.73386 & 0.00190 & 0.73576 \\
\hline $\mathrm{H}$ & 12 & 0.25850 & 0.00000 & 0.74053 & 0.00097 & 0.74150 \\
\hline $\mathrm{H}$ & 13 & 0.25197 & 0.00000 & 0.74711 & 0.00092 & 0.74803 \\
\hline $\mathrm{H}$ & 14 & 0.25368 & 0.00000 & 0.74544 & 0.00088 & 0.74632 \\
\hline $\mathrm{N}$ & 15 & -0.61959 & 1.99928 & 5.58124 & 0.03906 & 7.61959 \\
\hline C & 16 & 0.72887 & 1.99908 & 3.24561 & 0.02644 & 5.27113 \\
\hline $\mathrm{N}$ & 17 & -0.36739 & 1.99903 & 5.34857 & 0.01979 & 7.36739 \\
\hline C & 18 & 0.08130 & 1.99885 & 3.89920 & 0.02065 & 5.91870 \\
\hline C & 19 & -0.21350 & 1.99904 & 4.20054 & 0.01391 & 6.21350 \\
\hline C & 20 & -0.22760 & 1.99905 & 4.21403 & 0.01452 & 6.22760 \\
\hline C & 21 & -0.01371 & 1.99908 & 3.99952 & 0.01511 & 6.01371 \\
\hline C & 22 & -0.23323 & 1.99905 & 4.21978 & 0.01440 & 6.23323 \\
\hline
\end{tabular}




$\begin{array}{rrrrrrr}\mathrm{C} & 23 & -0.20394 & 1.99905 & 4.18982 & 0.01507 & 6.20394 \\ \mathrm{H} & 24 & 0.26287 & 0.00000 & 0.73627 & 0.00086 & 0.73713 \\ \mathrm{H} & 25 & 0.25621 & 0.00000 & 0.74289 & 0.00090 & 0.74379 \\ \mathrm{H} & 26 & 0.25508 & 0.00000 & 0.74404 & 0.00088 & 0.74492 \\ \mathrm{H} & 27 & 0.26608 & 0.00000 & 0.73294 & 0.00098 & 0.73392 \\ \mathrm{C} & 28 & -0.70849 & 1.99946 & 4.70099 & 0.00804 & 6.70849 \\ \mathrm{H} & 29 & 0.26091 & 0.00000 & 0.73851 & 0.00058 & 0.73909 \\ \mathrm{H} & 30 & 0.25789 & 0.00000 & 0.74152 & 0.00059 & 0.74211 \\ \mathrm{H} & 31 & 0.25049 & 0.00000 & 0.74888 & 0.00062 & 0.74951 \\ \mathrm{Fe} & 32 & 0.67037 & 17.99668 & 7.27222 & 0.06073 & 25.32963 \\ \mathrm{Cl} & 33 & -0.32990 & 9.99970 & 7.31792 & 0.01227 & 17.32990 \\ \mathrm{Cl} & 34 & -0.31595 & 9.99970 & 7.30383 & 0.01242 & 17.31595 \\ \mathrm{Cl} & 35 & -0.30527 & 9.99969 & 7.29270 & 0.01287 & 17.30527 \\ =============================================================== \\ \text { Total } & * & 0.00000 & 81.98030 & 112.61789 & 0.40182 & 195.00000\end{array}$

